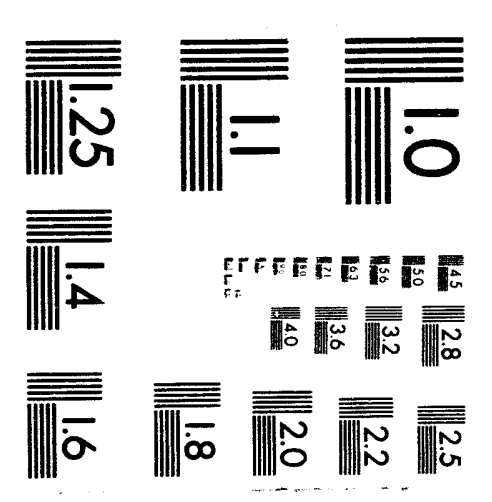



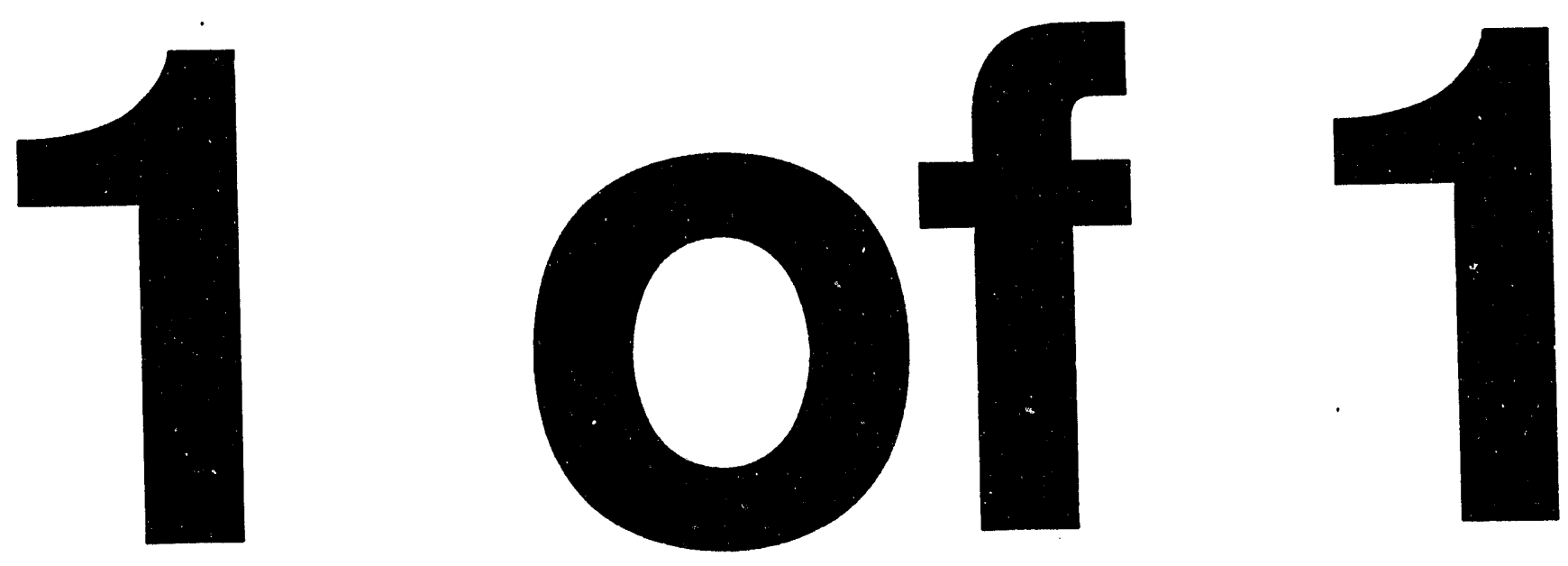
ORNL/TM-12722

Environmental Sciences Division

\section{RADIONUCLIDES IN SHALLOW GROUNDWATER \\ AT SOLID WASTE STORAGE AREA 5 NORTH, \\ OAK RIDGE NATIONAL LABORATORY}

Tom L. Ashwood and J. Daniel Marsh, Jr.

Environmental Sciences Division

Publication No. 4264

Date Published: April 1994

Prepared for

Office Environmental Management

(EW 312004 1)

Prepared by the

OAK RIDGE NATIONAL LABORATORY

Oak Ridge, Tennessee 37831

managed by

MARTIN MARIETTA ENERGY SYSTEMS, INC.

for the

U.S. DEPARTMENT OF ENERGY

under contract DE-AC05-84OR21400 


\section{CONTENTS}

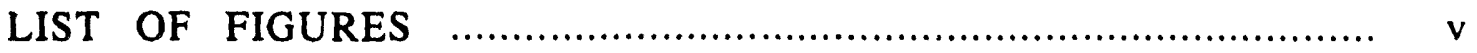

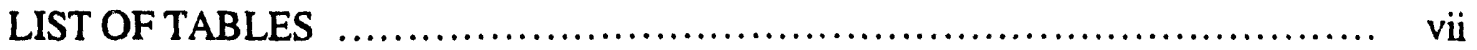

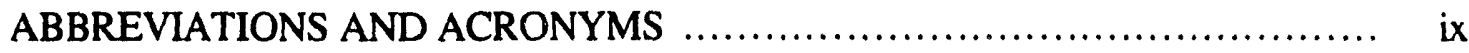

EXECUTIVE SUMMARY ……..............................................

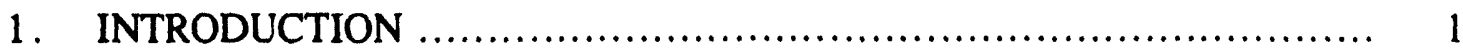

1.1 DESCRIPTION OF SOLID WASTE STORAGE AREA 5 NORTH ....... 1

1.2 REVIEW OF MONITORING RESULTS ............................. 1

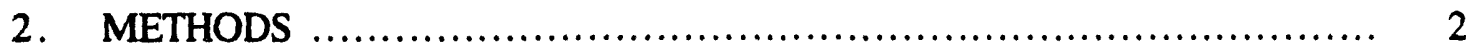

3. RESULTS AND DISCUSSION …..........................................

3.1 MONITORING WELLS ...........................................

3.2 SEEPS AND STREAM SAMPLES …................................ 3

3.3 WELL 516 GEOCHEMISTRY ....................................... 4

3.3.1 Methodology ..................................................... 5

3.3.2 Results and Discussion .................................... 5

3.3.3 Summary and Conclusions ..................................... 7

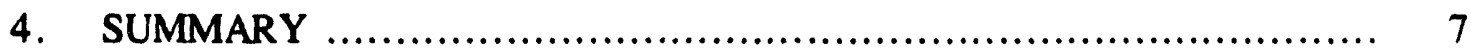

5. ACKNOWLEDGMENTS …................................................. 8

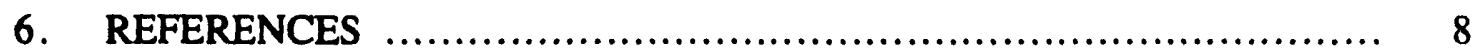

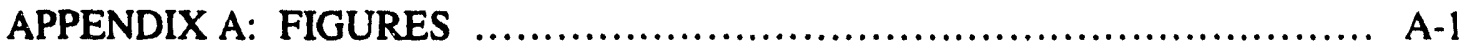

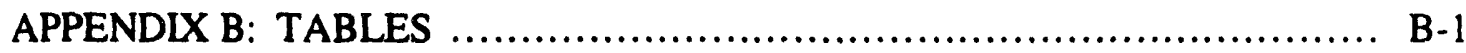

APPENDIX C: RELEVANT CORRESPONDENCE …........................ C-1 


\section{LIST OF TABLES}

Table

B.1 Current and historical radionuclide results from wells in and around SWSA 5 North

B.2 White Oak Creek bank seep and tributary monitoring results from SWSA 5 North

B.3 Radiological data from well 516 geochemical investigation B-15

B.4 Major cations and trace metals in well 516 geochemical investigation B-16

B .5 Anions and water chemistry data from well 516 geochemistry investigation

B.6 Ratios of $240 \mathrm{Pu}$ to ${ }^{244} \mathrm{Cm}$ and calculated migration times for well 516 in SWSA 5 North 


\section{LIST OF FIGURES}

Eigure

Page

A.1 Map of Solid Waste Storage Area 5 North showing transuranic waste facilities, monitoring wells, and samnling sites A-3

A.2 Gross alpha and ${ }^{244} \mathrm{Cm}$ activity in well 516 from November 1989 through April 1993

A.3 Curium-244, ${ }^{241} \mathrm{Am}$, and dissolved organic carbon as a function of particle size in well 516

A-5 


\section{ABBREVIATIONS AND ACRONYMS}

ASEMP Active Sites Environmental Monitoring Program

BNI Bechtel National, Inc.

DOE U.S. Department of Energy

LLW Low-level waste

ORNL Oak Ridge National Laboratory

SWSA Solid Waste Storage Area

TRU Transuranic

WOC White Oak Creek 


\section{EXECUTIVE SUMMARY}

This report presents a compilation of groundwater monitoring data collected between November 1989 and September 1983 from Solid Waste Storage Area (SWSA) 5 North at Oak Ridge National Laboratory. Monitoring data were collected as part of the Active Sites Environmental Monitoring Program that was implemented in 1989 in response to DOE Order 5820.2A.

SWSA 5 North was established for the retrievable storage of transuranic (TRU) wastes in 1970. Four types of storage have been used within SWSA 5 North: bunkers, vaults, wells, and trenches.

The fenced portion of SWSA 5 North covers $\sim 3.7$ ha ( 9 acres) in the White Oak Creek watershed south of ORNL. The area is bounded by White Oak Creek and two ephemeral tributaries of White Oak Creek.

Since 1989, groundwater has been monitored in wells around SWSA 5 North. During that time, elevated gross alpha contamination (reaching as high as $210 \mathrm{~Bq} / \mathrm{L}$ ) has consistently been detected in well 516. This well is adjacent to burial trenches in the southwest corner of the area. Water level measurements in wells 516 and 518 suggest that water periodically inundates the bottom of some of those trenches.

Virtually all of the gross alpha contamination is generated by ${ }^{244} \mathrm{Cm}$ and ${ }^{241} \mathrm{Am}$. A special geochemical investigation of well 516 suggests that nearly all of the ${ }^{244} \mathrm{Cm}$ and ${ }^{241} \mathrm{Am}$ is dissolved or associated with dissolved organic material.

Curium-244 and ${ }^{241} \mathrm{Am}$ are being transported at the rate of $\sim 2 \mathrm{~m} /$ year from the burial trenches, through well 516, to White Oak Creek, where ${ }^{244} \mathrm{Cm}$ has been detected in a few bank seeps. Concentrations at these seeps are near detection levels $(<1 \mathrm{~Bq} / \mathrm{L})$. 


\section{INTRODUCTION}

This report presents a compilation of groundwater monitoring data from Solid Waste Storage Area (SWSA) 5 North at Oak Ridge National Laboratory (ORNL). The purpose of this report is to provide a single reference for the history of monitoring results from SWSA 5 North as requested by U.S. Department of Energy (DOE) staff (Radcliffe 1993 and Frye 1993: in Appendix A of this report).

Monitoring data were collected as part of the Active Sites Environmental Monitoring Program (ASEMP) that was implemented in 1989 in response to DOE Order 5820.2A. All of these data have been reported in a series of ASEMP reports (Wickliff et al. 199 la,b; Ashwood et al. 1991, 1992a; Morrissey et al. 1994). Data from SWSA 5 North have also been presented at two national meetings (Ashwood et al. 1990; Marsh et al. 1993).

\subsection{DESCRIPTION OF SOLID WASTE STORAGE AREA 5 NORTH}

The fenced portion of SWSA 5 North covers $\sim 3.7$ ha (9 acres) in the White Oak Creek (WOC) watershed south of ORNL. The area is bounded by WOC and two ephemeral tributaries (Fig. A.1).

SWSA 5 North was established for the retrievable storage of transuranic (TRU) wastes in 1970 (Stewart et al. 1989). Four types of storage have been used within SWSA 5 North: bunkers, vaults, wells, and trenches.

'The bunker, Building 7855 (Fig. A.1), is an earth-sheltered facility composed of storage cells in which remote-handled TRU waste $(>200 \mathrm{mR} / \mathrm{h}$ at the surface) is stored in concrete casks. The building has sumps that are monitored by ASEMP.

Buildings 7826 and 7834 (Fig. A.1) are concrete block structures built $\sim 85 \%$ below grade. Each facility contains 24 storage vaults, which were previously used for storage of contact-handled TRU waste ( $<200 \mathrm{mR} / \mathrm{h}$ at the surface) but are now being closed under the Resource Conservation and Recovery Act. As a result of the closure process, all waste has been removed. If these buildings are used in the future for storage of radioactive wastes, their sumps will be monitored by ASEMP.

Storage wells at 7827 and 7829 (Fig. A.1) are -20 -ft deep with varying diameters.

The wells are encased in concretc and fitted with removable concrete plugs. A majority of the waste containers in these wells contain high-activity waste (spent nuclear fuel material) that is not considered remote-handled TRU waste (Stewart et al. 1989).

Approximately 275 waste containers are stored in 27 trenches at SWSA 5 North (Stewart et al. 1989). The majority of the containers are concrete casks, but 72 boxes and 2 drums are also listed in waste records (Stewart et al. 1989). Trenches are located in two areas of SWSA 5 North (Fig. A.1).

\subsection{REVIEW OF MONITORING RESULTS}

The Active Sites Environmental Monitoring Program, initiated in 1989, provides early detection and performance monitoring of TRU waste and active low-level waste (LLW) facilities at ORNL in accordance with DOE Order 5820.2A (Ashwood et al. 1992b). ORNL's Environmental Sciences Division implements the program for the Laboratory's Waste Management and Remedial Actions Division. 
ASEMP monitoring of SWSA 5 North began in November 1989. The first sample collected from well 516 (Fig. A.1) contained elevated concentrations of alpha contamination (largely from ${ }^{244} \mathrm{Cm}$ and a small amount of $241 \mathrm{Am}$ ) and of beta contamination relative to other groundwater wells in the area (Wickliff et al. 1991a).

After subsequent monitoring confirmed that there was a continuing source of ${ }^{244} \mathrm{Cm}$ contamination in the area of well 516, an occurrence report was filed and a plan was developed to investigate the source and extent of the contamination (see Appendix D in Ashwood et al. 1991). Results of this investigation were documented in a letter report (Ashwood and Wickliff 1991: in Appendix A of this report) and in the mid-FY 1991 ASEMP report (Ashwood et al. 1991).

The 1991 investigation and subsequent monitoring strongly implicate one or more of the trenches adjacent to well 516 as the source of ${ }^{244} \mathrm{Cm}$ and ${ }^{241} \mathrm{Am}$ contamination. These isotopes are being transported via the shallow groundwater to WOC, although concentrations in seeps along WOC are very low.

In early FY 1993, a geochemical investigation of well 516 was conducted. The purpose of this investigation was to determine the chemical form of the ${ }^{244} \mathrm{Cm}$ and ${ }^{241} \mathrm{Am}$ present in the well. Knowledge of the chemical form is important in determining what, if any, remedial actions are appropriate. Knowledge of the chemical form also will provide insight into whether these isotopes are biologically available.

\section{METHODS}

In SWSA 5 North, there is evidence that transport through the shallow aquifer, especially along preferred pathways, is the most important contaminant pathway. Thus, in this area, monitoring activities focus on groundwater monitoring wells, seeps, and samples from small tributaries.

Fifteen groundwater wells surrounding the TRU-waste storage facilities (Fig. A.1) have been sampled quarterly through June 1993. After June 1993, sampling frequency was reduced to once per year during the wettest season (January-March). Annual sampling should be sufficient to track known contamination and to identify any new contaminant plumes. In order to avoid duplication of effort, the ASEMP samples for the third quarter of FY 1993 were collected by the Bechtel National, Inc., (BP'I) team that was collecting samples for a remedial investigation of Waste Area Grouping 5.

Well samples are collected after the wells have been purged. All samples are filtered through $0.45-\mu \mathrm{m}$ filters and then acidified to a $\mathrm{pH}<2$ with nitric acid. Prior to October 1990 , however, samples were acidified but not filtered. The change to filtration was made because sediment from the wells interfered with the gross alpha/beta analyses and the presence of sediment confounded the interpretation of results.

Analytes in well samples include gross alpha, gross beta, ${ }^{60} \mathrm{Co},{ }^{137} \mathrm{Cs},{ }^{241} \mathrm{Am}$, and ${ }^{244} \mathrm{Cm}$. Beginning in FY 1992, tritium analysis was limited to one sample per year because tritium concentrations have always been in the range of rainwater, tritium is not a contaminant of concern for this site, and tritium data have not provided substantial insight into the transport of TRU contaminants. 
Eight seep and stream locations are also sampled on the same frequency as the wells (Ashwood et al. 1992b) if water is present at the time of sample collection. Sample sites 5NNT 01 and 5NST 02 are located within small tributaries of WOC. Seeps identified as "WOC XXX" (Fig. A.1) are located in the bank of WOC; however, only four of those seeps (WOC 160, WOC 175, WOC 213, and WOC 255) are sampled regularly. The remaining two seeps are between SWSA 5 North and WOC. Seep samples are analyzed for the same set of parameters as are well samples, except that no seep samples have been analyzed for tritium since 1991 for the same reasons that tritium sample frequency has been reduced in well samples.

ORNL's Analytical Chemistry Division performed all analyses reported in this document, except for samples collected and analyzed by BNI.

\section{RESULTS AND DISCUSSION}

\subsection{MONITORING WELLS}

Well 516 continues to contain measurable quantities of ${ }^{241} \mathrm{Am}$ and ${ }^{244} \mathrm{Cm}$ in every sample (Table B.1). Although ${ }^{244} \mathrm{Cm}$ concentrations appeared to decline between March 1991 and March 1993, recent samples suggest that the decrease has leveled off (Fig. A.2).

Wells $514,521,708,715$, and 716 appeared to contain traces of $244 \mathrm{Cm}$ in November 1992 (Table B.1). However, subsequent samples from those wells have not contained measurable ${ }^{244} \mathrm{Cm}$. Well 523 appeared to contain traces of ${ }^{241} \mathrm{Am}$ and $244 \mathrm{Cm}$ in March 1993. The May 1993 sample that BNI collected was not analyzed for these isotopes.

Well 516 continues to contain elevated gross beta concentrations on a sporadic basis (Table B.1). The elevated gross beta concentration that BNI reported for the May 1993 sample from well 519 (Table B.1) has a high associated counting error that renders this value not significantly different from zero.

\subsection{SEEPS AND STREAM SAMPLES}

North Tributary samples continue to contain sporadic traces of ${ }^{241} \mathrm{Am}$ and ${ }^{244} \mathrm{Cm}$ (Table B.2). South Tributary frequently contains substantial gross alpha contamination (Table B.2); however, the gross alpha concentrations are not associated with measurable concentrations of ${ }^{241} \mathrm{Am}$ or ${ }^{244} \mathrm{Cm}$. On the other hand, the June 1993 sample from South Tributary contained measurable traces of both isotopes but only a trace of gross alpha activity. Gross beta concentrations in South Tributary exceeded action levels twice in FY 1993 (Table B.2).

Of the bank seeps, only WOC 255 consistently contains measurable quantities of ${ }^{244} \mathrm{Cm}$ (Table B.2). Reported concentrations of ${ }^{241} \mathrm{Am}$ in WOC 255 were consistent throughout FY 1993, but only the latest sample was significantly greater than zero. Seep WOC 213 had the highest concentration of ${ }^{244} \mathrm{Cm}$ (Table B.2), but there has not been sufficient flow for sample collection since 1991.

Seep 5NW01 contained measurable amounts of ${ }^{244} \mathrm{Cm}$ throughout FY 1992 and 1993 (Table B 2), although there was sufficient flow in this seep for sample collection on 
only three occasions during the 2-year period. The anomalous appearance of $241 \mathrm{Am}$ in the April 1992 sample from seep 5NW02 was not repeated in any subsequent samples.

\subsection{WELL 516 GEOCHEMISTRY}

The objective of this study was to determine the mode and rate of physicochemical transport of ${ }^{244} \mathrm{Cm}$ and ${ }^{241} \mathrm{Am}$ from the waste trenches in SWSA 5 North to well 516. Quarterly well monitoring at this well for two and a half years has revealed ${ }^{244} \mathrm{Cm}$ and ${ }^{241} \mathrm{Am}$ activities ranging from 0.36 to $220 \mathrm{~Bq} / \mathrm{L}$ and 0.02 to $7 \mathrm{~Bq} / \mathrm{L}$, respectively (Morrissey et al. 1994). These activities are much higher than those for other wells in the immediate vicinity and provide a unique opportunity to study the geochemistry and transport of these two actinide elements. Determining the physicochemical form of these actinide elements in groundwater is a necessary first step in assessing their transport from SWSA 5 North and will provide valuable data for formulating successful remediation strategies.

Three different tasks were completed to accomplish this objective.

Task 1: Determine the chemistry of the groundwater environment to understand its effect on the physicochemical properties of the actinide elements.

The speciation of any radionuclide is a function of its surrounding environment. The $\mathrm{pH}$, redox conditions, degree of mineral saturation, and dissolved organic carbon, for example, all play a role in determining the radionuclide's oxidation state, solubility, complexation, and adsorption properties, which then determine the fate of the radionuclide.

Task 2: Separate any colloids present into particle size fractions to determine the distribution of the alpha activity by particle size.

Radionuclides associated with colloidal particles will be transported differently than if they were dissolved in solution. Transport of contaminants adsorbed onto colloids will occur at different rates and will follow different spatial flow paths than would the same radionuclides transported in the dissolved state. Colloids will be restricted to fractures and larger interconnected pore spaces, whereas dissolved ions can permcate the aquifer matrix. Stable colloids can migrate rapidly through zones of preferred flow and may be more readily mobilized by changes in hydrologic conditions.

Task 3: Estimate, on the basis of the radioactive decay schemes, the migration rate of ${ }^{244} \mathrm{Cm}$ and ${ }^{241} \mathrm{Am}$ from SWSA 5 North.

Curium-244 has a convenient half-life (18.11 years) with respect to the age of the waste trenches, and the buildup of the daughter product $(240 \mathrm{Pu})$ is dependent on this halflife. Therefore, the ${ }^{240} \mathrm{Pu} / 244 \mathrm{Cm}$ ratio acts as a clock, and the in-growth time will be a measure of the migration time of the ${ }^{244} \mathrm{Cm}$. (See the decay scheme.) This technique did not work as well for the ${ }^{241} \mathrm{Am} /{ }^{237} \mathrm{~Np}$ pair because of the long half-life for ${ }^{241} \mathrm{Am}$ (432 years) as compared with the age of the waste trenches.

Decay schemes for both ${ }^{244} \mathrm{Cm}$ and ${ }^{241} \mathrm{Am}$ are shown below.

$$
\left({ }^{244} \mathrm{Cm}\right) \frac{\alpha}{18.11 \mathrm{y}} \Rightarrow\left({ }^{240} \mathrm{Pu}\right) \frac{\alpha}{6560 \mathrm{y}} \Rightarrow\left({ }^{236} \mathrm{U}\right) \frac{\alpha}{23 \times 10^{10} \mathrm{y}} \Rightarrow\left({ }^{232} \mathrm{Th}\right) \Rightarrow 4 \mathrm{n} / \text { series }
$$




$$
\left({ }^{241} \mathrm{Am}\right) \frac{\alpha}{432 \mathrm{y}} \Rightarrow\left({ }^{237} \mathrm{~Np}\right) \frac{\alpha}{2.14 \times 10^{6} \mathrm{y}} \Rightarrow\left({ }^{233} \mathrm{~Pa}\right) \frac{\beta}{27 \mathrm{~d}} \Rightarrow\left({ }^{233} \mathrm{U}\right) \Rightarrow 4 \mathrm{n}+1 / \text { series }
$$

\subsubsection{Methodology}

Well 516 was sampled three different times beginning in December 1992 and ending in February 1993. The three sampling times were necessary to collect the different size fractions due to the slow hollow fiber filtration process. A peristaltic pump was used to collect groundwater from the 26.7 foot well. Depth to water was $\sim 9$ feet, and the well contained $\sim 18 \mathrm{ft}$ of water.

Measurements of $\mathrm{pH}$, Eh, temperature, dissolved oxygen, and conductivity were measured using a flow-through cell. The use of a flow-through cell minimizes the effect of oxygen on the redox sensitive measurements and avoids the introduction of artifacts that could unintentionally produce colloids such as the formation of colloidal ferric hydroxide. In addition to these, alkalinity by titration to $\mathrm{pH} 4.5$ and turbidity were also measured in the field. Tygon ${ }^{\circledR}$ tubing, previously washed with distilled/deionized (Milli-Q ${ }^{\circledR}$ ) water, was placed in the well $24 \mathrm{~h}$ in advance of sampling. This advanced installation allows for settling of particles that may have been disturbed from the sides of the well during tubing insertion. The pumping rate varied from 60 to 100 milliliters per minute (mL/min) to keep the drawdown of water in the well to a minimum. This is important in colloidal sampling because a faster flow rate would stir up particles from the sides of the well and could mobilize colloids that might not be present naturally.

The most direct means of identifying the importance of colloidal transport of radionuclides is the physical separation of colloidal particles by size filtration. To accomplish this separation at well 516, groundwater was passed through filters of $0.45 \mu \mathrm{m}$ (in-line), $0.1 \mu \mathrm{m}, 100,000(100 \mathrm{~K})$ dalton (unit of molecular weight), and 3,000 (3K) dalton, hollow fiber filters. These filters operate by tangential flow of the groundwater past a bundle of hollow fibers of a certain pore size. Only particles smaller than the pore size may then pass into the hollow fiber and be effectively removed from the bulk of the solution. Since the filters remove all particles below a certain size from the groundwater. the alpha activity for the particulate, greater than the filter size, is calculated by subtracting the alpha activity of the fraction from the alpha activity of an unfiltered portion of the groundwater. Metals, radionuclides, dissolved organic carbon, and anions were analyzed to determine the contribution of each size fraction to the concentrations of these analytes.

\subsubsection{Results and Discussion}

Tables B.3-B.5 present the data for well 516. Except for the radionuclides present. this well contained few contaminants. The groundwater in the well was mildly oxidizing with a $\mathrm{pH}$ of 6.5 to 6.8 and an ionic strength of 0.01 . The dominant cations were $\mathrm{Na}, \mathrm{Ca}$. $\mathrm{Mg}, \mathrm{Ba}$, and $\mathrm{Sr}$. Iron $(<0.3 \mathrm{mg} / \mathrm{L})$ and aluminum $(<0.2 \mathrm{mg} / \mathrm{L})$ were not detected were not present at sufficient levels to form natural colloids. The turbidity was also very low at 0.0 .5 NTU. This was the first indication that a process other than colloidal transport might be involved. The dominant anions were sulfate and bicarbonate, and chloride, fluoride, and nitrate were present in lesser amounts. The groundwater was supersaturated with respecl to barite $\left(\mathrm{BaSO}_{4}\right)$, which would remove by coprecipitation any naturally occurring radium and radon daughters. A gamma scan of the groundwater verified this by the absence of the ${ }^{214} \mathrm{~Pb}$ and $214 \mathrm{Bi}$ gamma lines at $352 \mathrm{KeV}$ and $609 \mathrm{KeV}$, respectively. 
Results of the colloidal sampling were somewhat surprising. Although colloids are a major means of contaminant transport at most waste sites around the country, no alpha activity was detected in the particulate fractions $>100 \mathrm{~K}$ daltons, and only $5 \%$ of the ${ }^{244} \mathrm{Cm}$ was found in the fraction between $3 \mathrm{~K}$ and $100 \mathrm{~K}$. That left $95 \%$ of the $244 \mathrm{Cm}$ and $100 \%$ of the ${ }^{241} \mathrm{Am}$ in the $<3 \mathrm{~K}$ fraction, which is considered to be the dissolved state (Fig. A.3). In addition, $95 \%$ of the dissolved organic carbon was also in the $<3 \mathrm{~K}$ fraction. This leads to the hypothesis that low molecular weight organics are complexing the radionuclides and keeping them in solution. If true, this has important implications for the hydrogeochemical modeling of contaminant transport from SWSA 5 North as well as for any type of remediation technique to be applied to this site. Organically complexed radionuclides will not be easily removed by ion exchange or filtration techniques and may be more mobile than if adsorbed to colloidal particles. A pretreatment of strong oxidant and/or UV radiation may be a necessary first step to break up the organics before attempting removal by cation exchange. If remedial actions are considered for this site, further study would be needed to verify this hypothesis.

The ratio of the accivity of a daughter radionuclide $(240 \mathrm{Pu})$ to the activity of its parent $(244 \mathrm{Cm})$ can be used to determine the length of time that the parent has been present using the following equation (derived from Eq. 3.32 in Foster and Wright 1980):

$$
t=\frac{\ln \left[1+\left(\frac{\lambda_{1}-\lambda_{2}}{\lambda_{2}}\right) \frac{A_{2}}{A_{1}}\right]}{\lambda_{1}-\lambda_{2}},
$$

where $t$ is the time in years; $\lambda_{1}$ and $\lambda_{2}$ are the decay constants for ${ }^{244} \mathrm{Cm}(0.0383 /$ year $)$ and ${ }^{240} \mathrm{Pu}$ (1.055 x 10-4/year), respectively; and $A_{1}$ and $A_{2}$ are the current activities of ${ }^{244} \mathrm{Cm}$ and $240 \mathrm{Pu}$, respectively.

Several assumptions are inherent in determining the migration rate on the basis of the $240 \mathrm{Pu} / 244 \mathrm{Cm}$ ratios. The primary assumption is that any plutonium buried at SWSA 5 North is in an insoluble form and is not being leached at any appreciable rate. This is the most critical assumption and the one on which the second and third assumptions depend. Although various isotopes of plutonium $(238,239,240$, and 241$)$ were disposed in the burial trenches (Stewart et al. 1989), this is still a reasonable assumption, since most waste trenches have a reducing environment in which plutonium is insoluble. This assumption is supported by the low levels of ${ }^{238} \mathrm{Pu}(<0.4 \mathrm{pCi} / \mathrm{L})$ observed in the groundwater. Second, since the alpha energies of ${ }^{239} \mathrm{Pu}$ and ${ }^{240} \mathrm{Pu}$ cannot be separated by alpha spectroscopy, their activities are reported together, and the assumption is made that all the $239 / 240 \mathrm{Pu}$ activity is due to only ${ }^{240} \mathrm{Pu}$, the daughter of $244 \mathrm{Cm}$. In other words, no ${ }^{239} \mathrm{Pu}$ is present. Third, all the ${ }^{240} \mathrm{Pu}$ observed was produced by the decay of ${ }^{244} \mathrm{Cm}$ while in transit from the waste trenches to well 516 . And finally, it is assumed that the $240 \mathrm{Pu}$ migrates concurrently with the ${ }^{244} \mathrm{Cm}$.

Given these assumptions, the $240 \mathrm{Pu} / 244 \mathrm{Cm}$ ratios at well 516 for the three sampling periods are given in Table B.6. The three times calculated for the measured ratios are remarkably similar and have an average of $7.5 \pm 1.1$ years. Since the distance from the nearest trenches to well 516 is $\sim 15$ meters, this gives a migration rate of $\sim 2 \mathrm{~m} /$ year, which is a moderate migration rate that is consistent with the saprolitic geology found at this site. 


\subsubsection{Summary and Conclusions}

Groundwater was characterized in well 516 at SWSA 5 North. Curium-244 and 241 Am were the only contaminants found. The groundwater was filtered for different colloidal size fractions, but most of the alpha activity was found in the dissolved state $(<3 \mathrm{~K}$ fraction). Most of the dissolved organic carbon was also in the dissolved state. This led to the hypothesis that the radionuclides are complexed by low $(<3 \mathrm{~K})$ molecular weight organics and are being transported in solution. This has important implications for the hydrogeochemical modeling of contaminant transport from SWSA 5 North as well as for any type of remediation technique to be applied to this site. On the basis of the $240 \mathrm{Pu} / 244 \mathrm{Cm}$ ratio, a migration rate for ${ }^{244} \mathrm{Cm}$ was calculated to be $\sim 2 \mathrm{~m} /$ year. Given this rate and the 20 years the waste trenches have been in place, the contaminants would have moved about $40 \mathrm{~m}$-much more than the $15 \mathrm{~m}$ needed to reach well 516 .

\section{SUMMARY}

Reported results from well 516 vary widely. Curium-244, for instance, varies from $220 \mathrm{~Bq} / \mathrm{L}$ in March 1991 to $0.36 \mathrm{~Bq} / \mathrm{L}$ in September 1992 (Table B.1). Although ${ }^{244} \mathrm{Cm}$ concentrations appear to be decreasing from 1991 levels, the most recent ASEMP results indicate that the decrease is leveling off. As we have suggested earlier (Wickliff et al. 1991a), water table elevation may control the leaching of TRU material from the buried waste. At higher water table elevations, more waste in the burial trenches is directly exposed to groundwater. If buried waste in contact with the water table is indeed the source of $244 \mathrm{Cm}$, then any remedial action must address either removal of the waste or isolation of the waste from the water table. Simply capping the trenches to eliminate infiltration may not prevent groundwater from contacting the buried waste.

Sporadic reporting of traces of ${ }^{241} \mathrm{Am}$ and ${ }^{244} \mathrm{Cm}$ in wells other than 516 should not be interpreted as a spreading of the contaminant plume. Reported values are so close to the detection limits and so random in time and location that the most likely explanation is analytical anomalies rather than true measurement of these isotopes.

Detection of traces of ${ }^{244} \mathrm{Cm}$ in seep 5NW01 in three successive sampling events over a 15-month period suggests that this seep may be occasionally discharging water from the same plume that passes through well 516. Seep 5NW01 is along strike and in a direct line with the burial trenches, well 516, and seep WOC 255 (Fig. A.1).

Bank seep WOC 255 continues to discharge ${ }^{244} \mathrm{Cm}$ and probably ${ }^{241} \mathrm{Am}$ into WOC. The flow volume is small, and the concentrations are quite low $\left(<0.8 \mathrm{~Bq} / \mathrm{L}{ }^{244} \mathrm{Cm}\right.$ and $\left.<0.02 \mathrm{~Bq} / \mathrm{L}{ }^{241} \mathrm{Am}\right)$.

Elevated gross alpha concentrations in South Tributary are apparently not due to TRU isotopes. ASEMP data indicate that virtually all alpha activity from SWSA 5 North is due to ${ }^{244} \mathrm{Cm}$. Thus, the absence of this isotope in South Tributary suggests that the alpha activity in this stream is not from SWSA 5 North. Traces of ${ }^{241} \mathrm{Am}$ and ${ }^{244} \mathrm{Cm}$ were detected in South Tributary for the first time during March 1993. Further monitoring is needed before we can determine whether these results are analytical anomalies or real indications of contamination. 


\section{ACKNOWLEDGMENTS}

Diana Hicks was instrumental in developing the initial monitoring program and in guiding the sample collection activities. John McCarthy and Tonia Mehlhorn provided ultrafiltration equipment and expertise. Della Marshall and Tony Thomas collected samples in the field and prepared the samples for analysis. Samples for FY 1993 were collected by the Bechtel National, Inc., team. Diana Hicks and Jerry Cunningham reviewed a draft copy of this report.

\section{REFERENCES}

Ashwood, T. L., D. S. Wickliff, C. M. Morrissey, and H. L. Adair. 1990. Active sites monitoring at Oak Ridge National Laboratory. pp. 397-399. In Proceedings of SPECTRUM 90 Nuclear and Hazardous Waste Management International Topical Meeting, September 30-October 4, 1990, Knoxville, Tennessee. American Nuclear Society, La Grange Park, Ill.

Ashwood, T. L., D. S. Wickliff, and C. M. Morrissey. 1991. Active sites environmental monitoring program: Mid-FY 1991 report. ORNL/M-1442. Oak Ridge National Laboratory.

Ashwood, T. L., D. S. Wickliff, and C. M. Morrissey. 1992a. Active sites environmental monitoring program: FY 1991 report. ORNL/M-1792. Oak Ridge National Laboratory.

Ashwood, T. L., D. S. Wickliff, and C. M. Morrissey. 1992b. Active sites environmental monitoring program: Program plan, Rev. 1. ORNL/M-1793. Oak Ridge National Laboratory.

DOE (U.S. Department of Energy). 1988. Radioactive Waste Management. DOE Order $5820.2 A, 9 / 26 / 88$. Washington, D.C.

Foster, A. R., and R. L. Wright, Jr. 1980. Basic Nuclear Engineering. Allyn and Bacon, Inc., Boston.

Marsh, J. D., Jr., T. L. Ashwood, and D. S. Hicks. 1993. Geochemical factors influencing the occurrence of Cm-244 and Am-241 in groundwater at a shallow waste burial site at Oak Ridge National Laboratory. Abstract GEOC 131 In Book of Abstracts, 205th American Chemical Society National Meeting, March 28-April 2, 1993, Denver. American Chemical Socicty, Washington, D.C.

Morrissey, C. M., T. L. Ashwood, and D. S. Hicks. 1994. Active sites environmental monitoring program: FY 1992 annual report. ORNL/M-3183. Oak Ridge National Laboratory.

Stewart, R. C., L. S. Dickerson, S. F. Joost, and D. C. Osucha. 1989. Remote-handled transuranic solid waste characterization study: Oak Ridge Naticnal Laboratory. ORNL/TM-11050. Oak Ridge National Laboratory.

Wickliff, D. S., C. M. Morrissey, and T. L. Ashwood. 1991a. Active sites environmental monitoring program: Mid-FY 1990 summary report. ORNL/M-1179. Oak Ridge National Laboratory.

Wickliff, D. S., C. M. Morrissey, and T. L. Ashwood. 1991b. Active sites environmental monitoring program: FY 1990 report. ORNL/M-1327. Oak Ridge National Laboratory. 
APPENDIX A

FIGURES

A-1 
ORNL-DWG 91-15062 R2

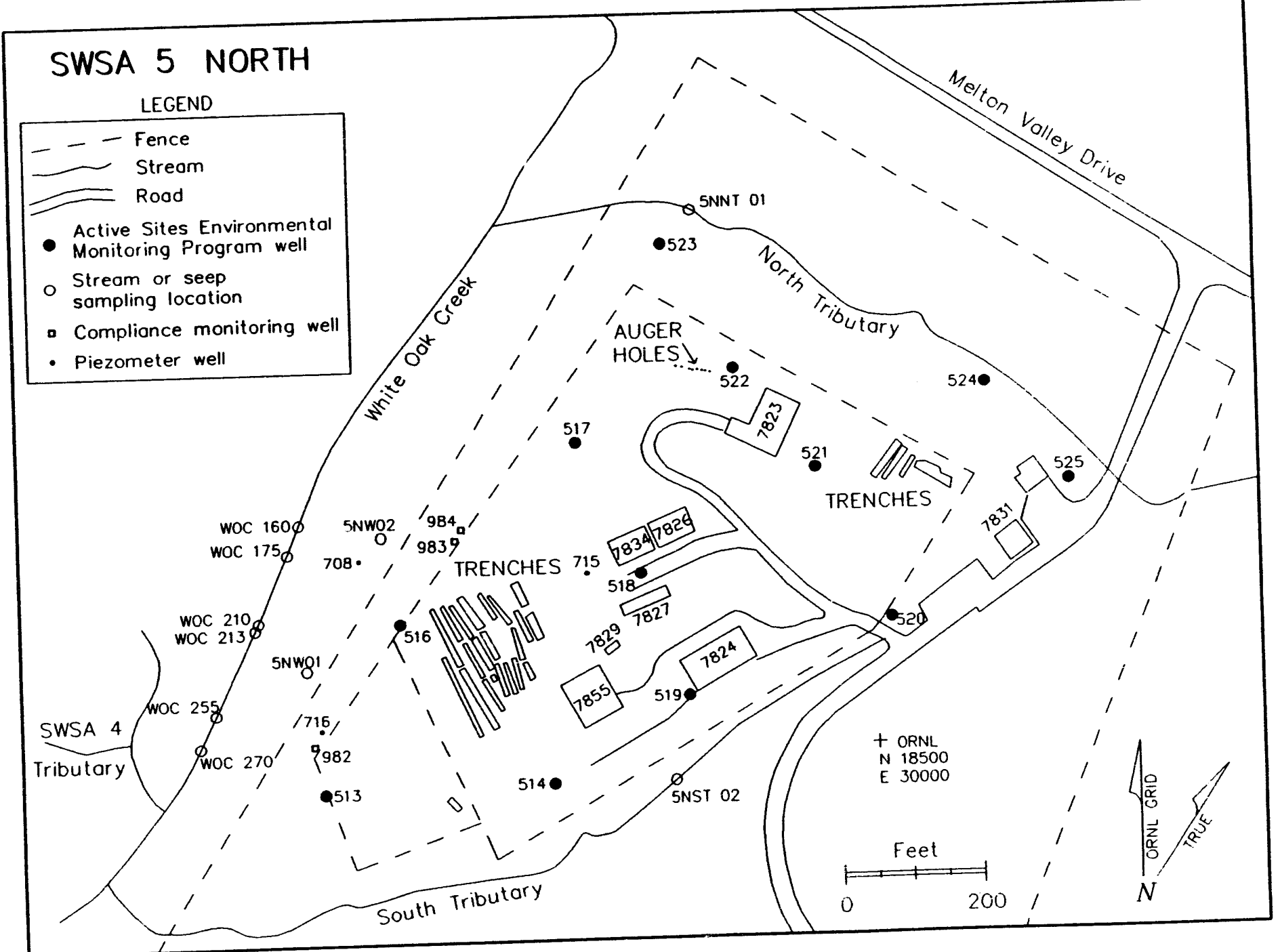

Map of Solid Waste Area 5 North showing transuranic waste facilities, monitoring wells, and sampling sites. 
hull. 


Am-244 $\quad$ Dissolved organic carbon

ORNL-DWG 94M-1271

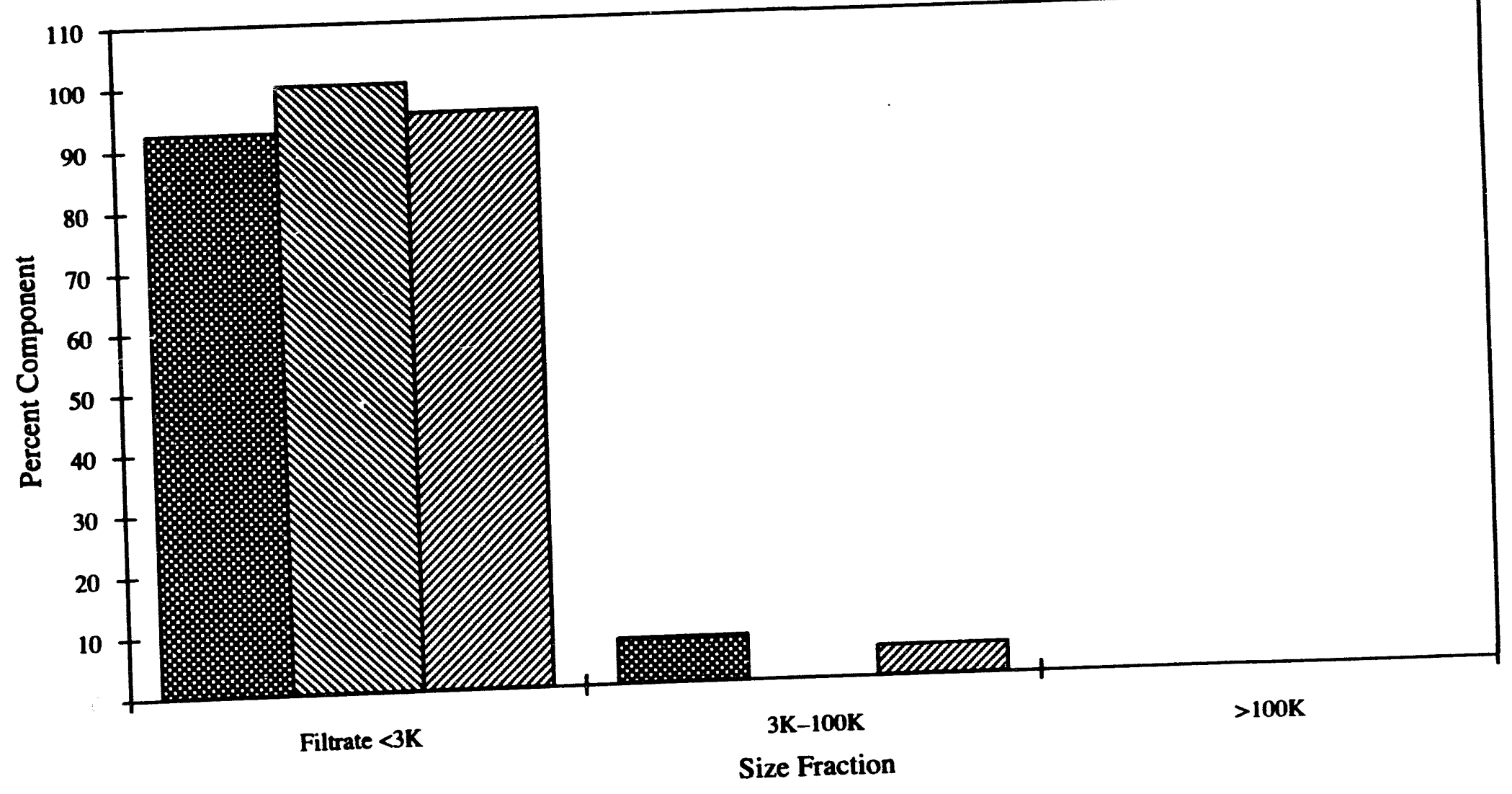

Fig. A.3. Curium-244, ${ }^{241} \mathrm{Am}$, and dissolved organic carbon as a function of particle size in well 516. 


\section{APPENDIX B}

TABLES

B-1 
Table B.1. Current and historical radionuclide results from wells in and around SWSA 5 North ${ }^{a}$

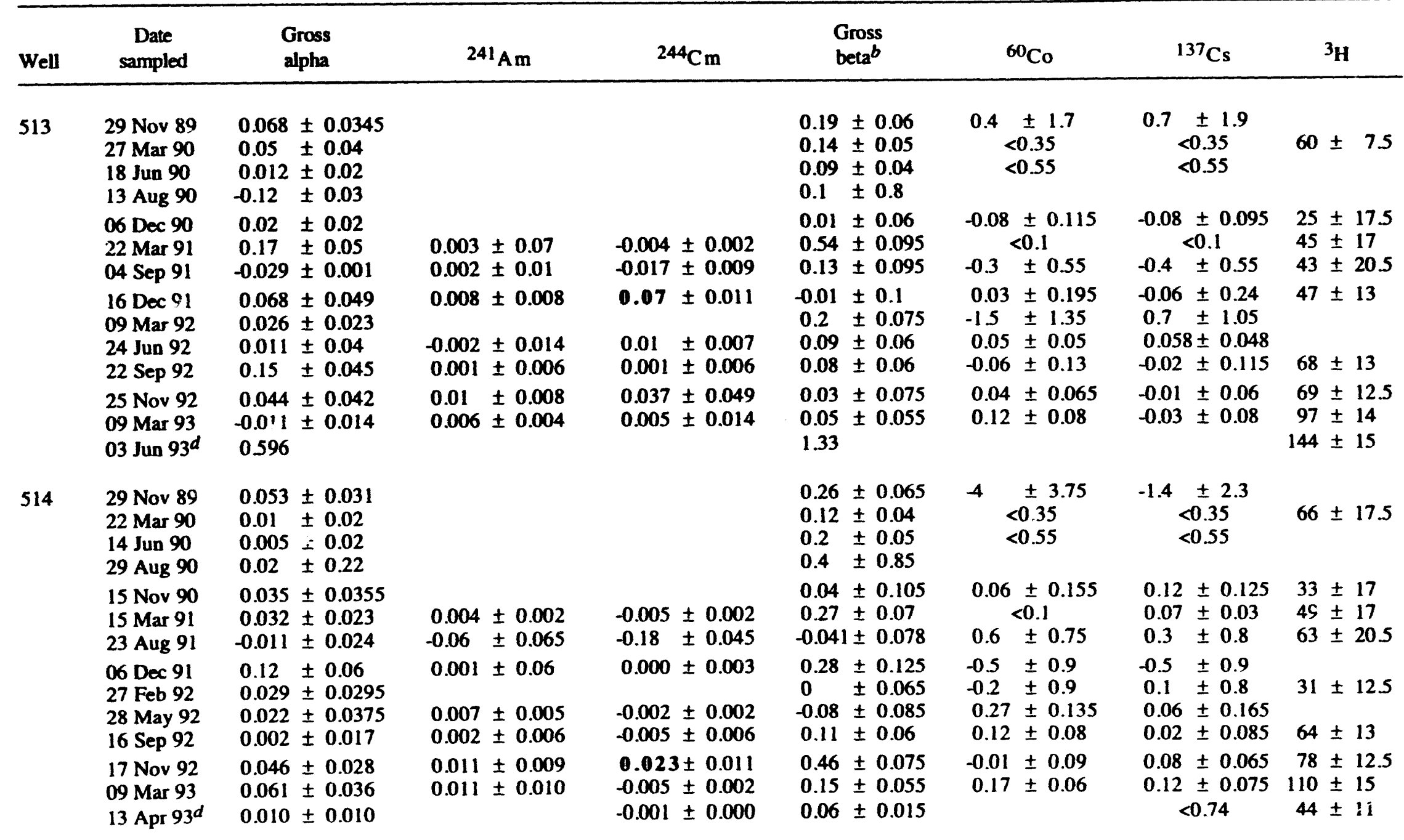


Table B.1 (continued)

\begin{tabular}{|c|c|c|c|c|c|c|c|c|c|c|c|}
\hline Well & $\begin{array}{c}\text { Date } \\
\text { sampled }\end{array}$ & & $\begin{array}{l}\text { Gross } \\
\text { alpha }\end{array}$ & ${ }^{241} \mathrm{Am}$ & & ${ }^{4} \mathrm{Cm}$ & & $\begin{array}{l}\text { Gross } \\
\text { beta }^{b}\end{array}$ & ${ }^{60} \mathrm{Co}$ & ${ }^{137} \mathrm{Cs}$ & ${ }^{3} \mathbf{H}$ \\
\hline \multirow[t]{4}{*}{516} & $\begin{array}{l}29 \text { Nov } 89 \\
28 \text { Mar } 90 \\
26 \text { Jun } 90 \\
26 \text { Jul } 90 \\
16 \text { Aug } 90\end{array}$ & $\begin{array}{c}170 \\
79 \\
9.3 \\
120 \\
110\end{array}$ & $\begin{array}{l} \pm 10 \\
\pm 3 \\
\pm 0.23 \\
\pm 5 \\
\pm 5\end{array}$ & $\begin{array}{l}5.53^{c} \pm 0.21 \\
0.66 \pm 0.025\end{array}$ & $\begin{array}{c}140 \\
71.1 \\
43\end{array}$ & $\begin{array}{l} \pm 5 \\
\pm 2.7 \\
\pm 0.5\end{array}$ & $\begin{array}{l}13 \\
2.68 \\
7.3\end{array}$ & $\begin{array}{l} \pm 1.5 \\
\pm 0.14 \\
\pm 0.35\end{array}$ & $\begin{array}{c}0.15 \pm 0.085 \\
0.8 \pm 1.2 \\
<0.55\end{array}$ & $\begin{array}{c}0.01 \pm 0.095 \\
1.1 \pm 1.05 \\
<0.55\end{array}$ & $40 \pm 17$ \\
\hline & $\begin{array}{l}13 \text { Nov } 90 \\
22 \text { Mar } 91 \\
04 \text { Sep } 91\end{array}$ & $\begin{array}{r}38 \\
210 \\
46\end{array}$ & $\begin{array}{l} \pm 1 \\
\pm 5 \\
\pm 1\end{array}$ & $\begin{array}{ll}1.7 & \pm 0.25 \\
7 & \pm 0.5 \\
0.99 & \pm 0.115\end{array}$ & $\begin{array}{r}41 \\
220 \\
54\end{array}$ & $\begin{array}{l} \pm 1 \\
\pm 5 \\
\pm 1\end{array}$ & $\begin{array}{l}2.2 \\
15 \\
3.8\end{array}$ & $\begin{array}{l} \pm 0.25 \\
\pm 0.5 \\
\pm 0.2\end{array}$ & $\begin{aligned}-0.02 & \pm 0.295 \\
0.07 & \pm 0.05 \\
0.6 & \pm 0.7\end{aligned}$ & $\begin{array}{l}0.14 \pm 0.22 \\
0.08 \pm 0.05 \\
0.7 \pm 0.6\end{array}$ & $\begin{array}{l}51 \pm 12 \\
35 \pm 16.5 \\
-49 \pm 1\end{array}$ \\
\hline & $\begin{array}{l}18 \operatorname{Dec} 91 \\
30 \text { Jun } 92 \\
28 \text { Sep } 92\end{array}$ & $\begin{array}{l}24 \\
14 \\
6.4\end{array}$ & $\begin{array}{l} \pm 0.5 \\
\pm 0.5 \\
\pm 0.3\end{array}$ & $\begin{array}{l}0.62 \pm 0.03 \\
0.18 \pm 0.02 \\
0.019 \pm 0.007\end{array}$ & $\begin{array}{l}24 \\
15 \\
0.36\end{array}$ & $\begin{array}{l} \pm 0.5 \\
\pm 0.5 \\
\pm 0.015\end{array}$ & $\begin{array}{l}1.6 \\
0.99 \\
0.71\end{array}$ & $\begin{array}{l} \pm 0.15 \\
\pm 0.145 \\
\pm 0.1\end{array}$ & $\begin{array}{ll}-0.01 & \pm 0.38 \\
0.1 & \pm 0.105 \\
0.04 & \pm 0.09\end{array}$ & $\begin{array}{l}-0.01 \pm 0.3 \\
0.07 \pm 0.1 \\
0.02 \pm 0.07\end{array}$ & $\begin{array}{l}36 \pm 13 \\
49 \pm 12\end{array}$ \\
\hline & $\begin{array}{l}09 \text { Nov } 92 \\
25 \text { Mar } 93 \\
30 \text { Apr } 93^{d} \\
30 \text { Apr } 93^{e}\end{array}$ & $\begin{array}{r}5.0 \\
7.0 \\
15.9 \\
13.2\end{array}$ & $\begin{array}{l} \pm 0.25 \\
\pm 0.35 \\
\pm 3.5 \\
\pm \mathbf{0 . 4 0}\end{array}$ & $\begin{array}{l}0.057 \pm 0.010 \\
0.39 \pm 0.025 \\
0.407 \pm 0.081 \\
0.14 \pm 0.048\end{array}$ & $\begin{array}{l}7.1 \\
6.2 \\
9.26 \\
12.3\end{array}$ & $\begin{array}{l} \pm 0.1 \\
\pm 0.1 \\
\pm 1.15 \\
\pm 2.13\end{array}$ & $\begin{array}{c}1.3 \\
0.58 \\
14.8 \\
1.25\end{array}$ & $\begin{array}{l} \pm 0.1 \\
\pm 0.085 \\
\pm 2.26 \\
\pm 0.13\end{array}$ & $\begin{array}{r}-0.04 \pm 0.08 \\
0.24 \pm 0.11\end{array}$ & $\begin{array}{c}0.03 \pm 0.06 \\
0.07 \pm 0.12 \\
<0.63 \\
<0.56\end{array}$ & $56 \pm 12$ \\
\hline \multirow[t]{4}{*}{517} & $\begin{array}{l}29 \text { Nov } 89 \\
06 \text { Mar } 90 \\
14 \text { Jun } 90 \\
29 \text { Aug } 90\end{array}$ & $\begin{array}{l}-0.21 \\
-0.001 \\
-0.002 \\
0.09\end{array}$ & $\begin{array}{l} \pm 0.04 \\
\pm 0.006 \\
\pm 0.01 \\
\pm 0.23\end{array}$ & & & & $\begin{array}{l}-3 \\
0.22 \\
0.09 \\
0.1\end{array}$ & $\begin{array}{l} \pm 0.7 \\
\pm 0.05 \\
\pm 0.03 \\
\pm 0.9\end{array}$ & $\begin{array}{c}-1.1 \leq 2.9 \\
<2.5 \\
<0.55\end{array}$ & 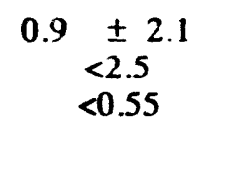 & $54 \pm 17.5$ \\
\hline & $\begin{array}{l}15 \text { Nov } 90 \\
20 \text { Mar } 91 \\
30 \text { Aug } 91\end{array}$ & $\begin{array}{l}0.023 \\
0 \\
0.004\end{array}$ & $\begin{array}{l} \pm 0.041 \\
\pm 0.029 \\
\pm 0.029\end{array}$ & $\begin{array}{l}-0.008 \pm 0.01 \\
-0.02 \pm 0.05\end{array}$ & $\begin{array}{l}-0.007 \\
-0.045\end{array}$ & $\begin{array}{l} \pm 0.008 \\
\pm 0.045\end{array}$ & $\begin{array}{l}0.28 \\
0.31 \\
0.51\end{array}$ & $\begin{array}{l} \pm 0.11 \\
\pm 0.075 \\
\pm 0.11\end{array}$ & $\begin{array}{c}-0.11 \pm 0.165 \\
<0.1 \\
-1 \quad \pm 2.05\end{array}$ & $\begin{array}{c}0.05 \pm 0.15 \\
<0.1 \\
0.2 \pm 1.35\end{array}$ & $\begin{array}{c}37 \pm 17 \\
1916.5 \\
9 \pm 20\end{array}$ \\
\hline & $\begin{array}{l}10 \text { Dec } 91 \\
27 \text { Feb } 92 \\
08 \text { Jun } 92 \\
17 \text { Sep } 92\end{array}$ & $\begin{array}{c}0.05 \\
-0.019 \\
0.019 \\
0.003\end{array}$ & $\begin{array}{l} \pm 0.05 \\
\pm 0.02 \\
\pm 0.047 \\
\pm 0.021\end{array}$ & $\begin{array}{l}0.001 \pm 0.008 \\
0.008 \pm 0.006 \\
0.015 \pm 0.008\end{array}$ & $\begin{array}{r}-0.003 \\
-0.008 \\
0.009\end{array}$ & $\begin{array}{l} \pm 0.006 \\
\pm 0.002 \\
\pm 0.006\end{array}$ & $\begin{array}{l}0.07 \\
0.24 \\
0.18 \\
0.06\end{array}$ & $\begin{array}{l} \pm 0.115 \\
4 \pm 0.085 \\
\pm \pm 0.105 \\
5 \pm 0.06\end{array}$ & $\begin{aligned} 1.2 & \pm 0.6 \\
0.37 & \pm 0.125 \\
-0.02 & \pm 0.175 \\
0.06 & \pm 0.09\end{aligned}$ & $\begin{aligned} 0.1 & \pm 0.75 \\
0.16 & \pm 0.485 \\
-0.02 & \pm 0.155 \\
0.06 & \pm 0.1\end{aligned}$ & $\begin{array}{l}35 \pm 13 \\
44 \pm 12\end{array}$ \\
\hline & $\begin{array}{l}19 \text { Nov } 92 \\
11 \text { Mar } 93 \\
09 \text { Jun } 93^{d}\end{array}$ & $\begin{array}{l}0.12 \\
-0.031 \\
0.60\end{array}$ & $\begin{array}{l} \pm 0.05 \\
\pm 0.024\end{array}$ & $\begin{array}{l}0.003 \pm 0.009 \\
0.001 \pm 0.006\end{array}$ & $\begin{array}{r}-0.006 \\
0.002\end{array}$ & $\begin{array}{l} \pm 0.008 \\
\pm 0.005\end{array}$ & $\begin{array}{l}0.33 \\
0.07 \\
1.33\end{array}$ & $\begin{array}{l}3 \pm 0.075 \\
\pm 0.06 \\
3\end{array}$ & $\begin{aligned} 0.07 & \pm 0.085 \\
-0.19 & \pm 0.18\end{aligned}$ & $\begin{aligned}-0.02 & \pm 0.085 \\
0.16 & \pm 0.09\end{aligned}$ & $\begin{array}{l}61 \pm 12 \\
83 \pm 13.5 \\
62 \pm 12\end{array}$ \\
\hline
\end{tabular}


Table B.1 (continued)

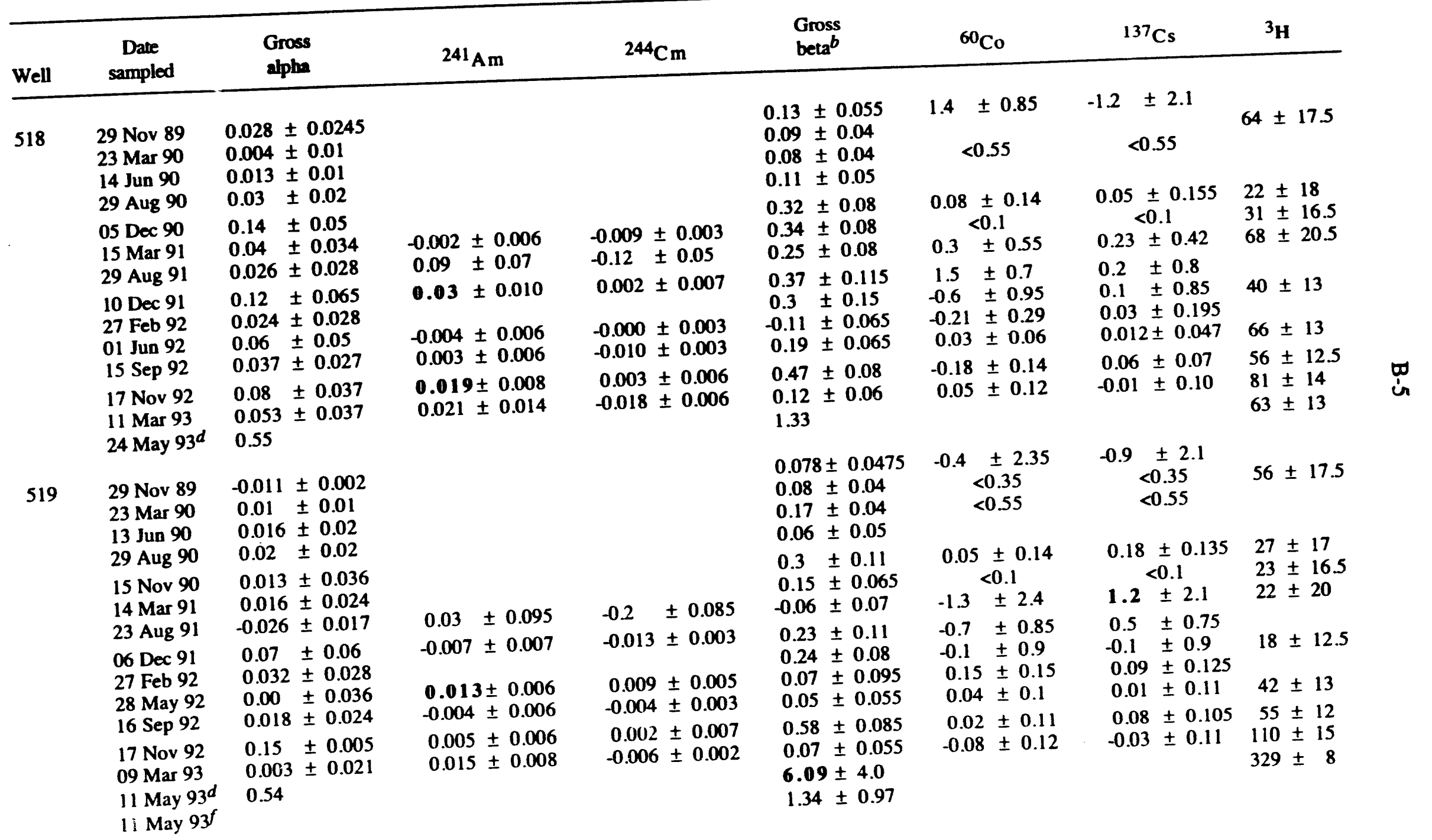


Table B.1 (continued)

\begin{tabular}{|c|c|c|c|c|c|c|c|c|c|c|}
\hline Well & $\begin{array}{c}\text { Date } \\
\text { sampled }\end{array}$ & & $\begin{array}{l}\text { Iross } \\
\text { Ipha }\end{array}$ & ${ }^{241} \mathrm{Am}$ & ${ }^{244} \mathrm{Cm}$ & & $\begin{array}{l}\text { Gross } \\
\text { beta } b\end{array}$ & ${ }^{60} \mathrm{Co}$ & ${ }^{137} \mathrm{Cs}$ & ${ }^{3} \mathbf{H}$ \\
\hline \multirow[t]{4}{*}{520} & $\begin{array}{l}29 \text { Nov } 89 \\
22 \text { Mar } 90 \\
13 \text { Jun } 90 \\
30 \text { Aug } 90\end{array}$ & $\begin{array}{l}0.02 \\
0.01 \\
0.007 \\
0.32\end{array}$ & $\begin{array}{l} \pm 0.0255 \\
\pm 0.02 \\
\pm 0.02 \\
\pm 0.32\end{array}$ & & & $\begin{array}{l}0.05 \\
0.08 \\
0.1 \\
0.8\end{array}$ & $\begin{array}{l} \pm 0.07 \\
\pm 0.04 \\
\pm 0.04 \\
\pm 0.85\end{array}$ & $\begin{array}{c}0.08 \pm 0.175 \\
<0.35 \\
<0.55\end{array}$ & $\begin{array}{r}0.010 .165 \\
<0.35 \\
<0.55\end{array}$ & $76 \pm 17.5$ \\
\hline & $\begin{array}{l}12 \text { Nov } 90 \\
20 \text { Mar } 91 \\
28 \text { Aug } 91\end{array}$ & $\begin{array}{l}0.05 \\
0.021 \\
0.09\end{array}$ & $\begin{array}{l} \pm 0.055 \\
\pm 0.027 \\
\pm 0.042\end{array}$ & $\begin{array}{c}-0.004 \pm 0.005 \\
0.08 \pm 0.06\end{array}$ & $\begin{array}{l}-0.007 \pm 0.002 \\
-0.12 \pm 0.035\end{array}$ & $\begin{array}{l}0.08 \\
0.19 \\
0.21\end{array}$ & $\begin{array}{l} \pm 0.1 \\
\pm 0.065 \\
\pm 0.08\end{array}$ & $\begin{array}{c}0.12 \pm 0.09 \\
<0.1 \\
-2.4 \pm 2.8\end{array}$ & $\begin{array}{c}0.02 \pm 0.09 \\
<0.1 \\
0.2 \pm 0.6\end{array}$ & $\begin{array}{l}36 \pm 12 \\
26 \pm 16.5 \\
9 \pm 20\end{array}$ \\
\hline & $\begin{array}{l}13 \text { Dec } 91 \\
05 \text { Mar } 92 \\
10 \text { Jun } 92 \\
28 \text { Sep } 92\end{array}$ & $\begin{array}{l}-0.005 \\
0.014 \\
0.12 \\
0.052\end{array}$ & $\begin{array}{l} \pm 0.027 \\
\pm 0.025 \\
\pm 0.04 \\
\pm 0.031\end{array}$ & $\begin{array}{r}0.002 \pm 0.008 \\
0.015 \pm 0.019 \\
-0.005 \pm 0.005\end{array}$ & $\begin{array}{l}-0.012 \pm 0.006 \\
0.11 \pm 0.02 \\
-0.001 \pm 0.005\end{array}$ & $\begin{array}{l}0.2 \\
0.1 \\
0.14 \\
0.14\end{array}$ & $\begin{array}{l} \pm 0.1 \\
\pm 0.07 \\
\pm 0.06 \\
\pm 0.065\end{array}$ & $\begin{array}{ll}-1.4 & \pm 1 \\
1.1 & \pm 0.8 \\
0.01 & \pm 0.1 \\
0.01 & \pm 0.07\end{array}$ & $\begin{array}{ll}-0.6 & \pm 0.85 \\
-0.4 & \pm 1.05 \\
0.02 & \pm 0.09 \\
0.01 & \pm 0.055\end{array}$ & $\begin{array}{l}23 \pm 12.5 \\
44 \pm 12.5\end{array}$ \\
\hline & $\begin{array}{l}17 \text { Nov } 92 \\
18 \text { Mar } 93 \\
10 \text { Jun } 93^{d} \\
10 \text { Jun } 93^{e}\end{array}$ & $\begin{array}{l}0.068 \\
0.06 \\
0.64 \\
0.55\end{array}$ & $\begin{array}{l} \pm 0.035 \\
\pm 0.036 \\
\pm 0.54\end{array}$ & $\begin{array}{r}-0.007 \pm 0.004 \\
0.002 \pm 0.016\end{array}$ & $\begin{array}{l}-0.000 \pm 0.004 \\
-0.008 \pm 0.012\end{array}$ & $\begin{array}{l}0.29 \\
0.17 \\
1.33 \\
1.33\end{array}$ & $\begin{array}{l} \pm 0.07 \\
\pm 0.065\end{array}$ & $\begin{array}{r}0.03 \pm 0.08 \\
-0.04 \pm 0.12\end{array}$ & $\begin{array}{l}0.05 \pm 0.07 \\
0.04 \pm 0.10\end{array}$ & $\begin{array}{r}53 \pm 12 \\
100 \pm 15 \\
197 \pm 16 \\
138 \pm 14\end{array}$ \\
\hline \multirow[t]{4}{*}{521} & $\begin{array}{l}29 \text { Nov } 89 \\
23 \text { Mar } 90 \\
18 \text { Jun } 90 \\
30 \text { Aug } 90\end{array}$ & $\begin{array}{l}0.014 \\
0.004 \\
0.01 \\
-0.15\end{array}$ & $\begin{array}{l} \pm 0.012 \\
\pm 0.017 \\
\pm 0.01 \\
\pm 0.035\end{array}$ & & & $\begin{array}{l}0.64 \\
0.21 \\
0.13 \\
1.2\end{array}$ & $\begin{array}{l} \pm 0.05 \\
\pm 0.05 \\
\pm 0.04 \\
\pm 0.9\end{array}$ & $\begin{array}{c}0.08 \pm 0.125 \\
<0.35 \\
<0.55\end{array}$ & $\begin{array}{c}0.2 \pm 0.07 \\
<0.35 \\
<0.55\end{array}$ & $28 \pm 17$ \\
\hline & $\begin{array}{l}05 \text { Dec } 90 \\
20 \text { Mar } 91 \\
30 \text { Aug } 91\end{array}$ & $\begin{array}{l}0 \\
-0.1 \\
0.84\end{array}$ & $\begin{array}{l} \pm 0.05 \\
\pm 0.13 \\
\pm 0.405\end{array}$ & $0.08 \pm 0.075$ & $0.3 \pm 0.09$ & $\begin{array}{l}0.12 \\
0.9 \\
1.1\end{array}$ & $\begin{array}{l} \pm 0.075 \\
\pm 0.6 \\
\pm 0.75\end{array}$ & $\begin{array}{c}0.04 \pm 0.12 \\
<0.1 \\
-1.6 \pm 2.95\end{array}$ & $\begin{array}{c}-0.04 \pm 0.12 \\
<0.1 \\
-1.4 \pm 2.65\end{array}$ & $\begin{aligned}-45 & \pm 16.5 \\
7 & \pm 16 \\
13 & \pm 20\end{aligned}$ \\
\hline & $\begin{array}{l}12 \text { Dec } 91 \\
\text { 05 Mar } 92 \\
28 \text { May } 92 \\
18 \text { Sep } 92\end{array}$ & $\begin{array}{l}0.16 \\
0.1 \\
-0.06 \\
0.1\end{array}$ & $\begin{array}{l} \pm 0.13 \\
\pm 0.1 \\
\pm 0.012 \\
\pm 0.07\end{array}$ & $\begin{array}{l}0.062 \pm 0.014 \\
0.006 \pm 0.006 \\
0.004 \pm 0.006\end{array}$ & $\begin{array}{l}0.002 \pm 0.01 \\
0.015 \pm 0.005 \\
0.002 \pm 0.006\end{array}$ & $\begin{array}{c}0.28 \\
-0.09 \\
1.4 \\
0.15\end{array}$ & $\begin{array}{l} \pm 0.23 \\
\pm 0.145 \\
\pm 0.035 \\
\pm 0.105\end{array}$ & $\begin{array}{ll}0.3 & \pm 0.8 \\
0.05 & \pm 0.475 \\
-0.05 & \pm 0.1 \\
-0.05 & \pm 0.11\end{array}$ & $\begin{array}{ll}-0.1 & \pm 0.85 \\
-0.13 & \pm 0.485 \\
-0.07 & \pm 0.1 \\
0.03 & \pm 0.085\end{array}$ & $\begin{array}{r}3 \pm 12 \\
30 \pm 12\end{array}$ \\
\hline & $\begin{array}{l}19 \text { Nov } 92 \\
18 \text { Mar } 93 \\
10 \text { May } 93^{d}\end{array}$ & $\begin{array}{r}0.04 \\
-0.02 \\
0.32\end{array}$ & $\begin{array}{l} \pm 0.037 \\
\pm 0.06 \\
\pm 0.21\end{array}$ & $\begin{array}{l}0.027 \pm 0.014 \\
0.006 \pm 0.016\end{array}$ & $\begin{array}{r}0.038 \pm 0.014 \\
-0.006 \pm 0.008\end{array}$ & $\begin{array}{l}0.20 \\
0.13 \\
0.63\end{array}$ & $\begin{array}{l} \pm 0.07 \\
\pm 0.065 \\
\pm 0.36\end{array}$ & $\begin{aligned}-0.01 & \pm 0.085 \\
0.08 & \pm 0.10\end{aligned}$ & $\begin{aligned}-0.01 & \pm 0.075 \\
0.01 & \pm 0.09\end{aligned}$ & $\begin{array}{r}31 \pm 12 \\
110 \pm 15 \\
60 \pm 5\end{array}$ \\
\hline
\end{tabular}


Table B.1 (continued)

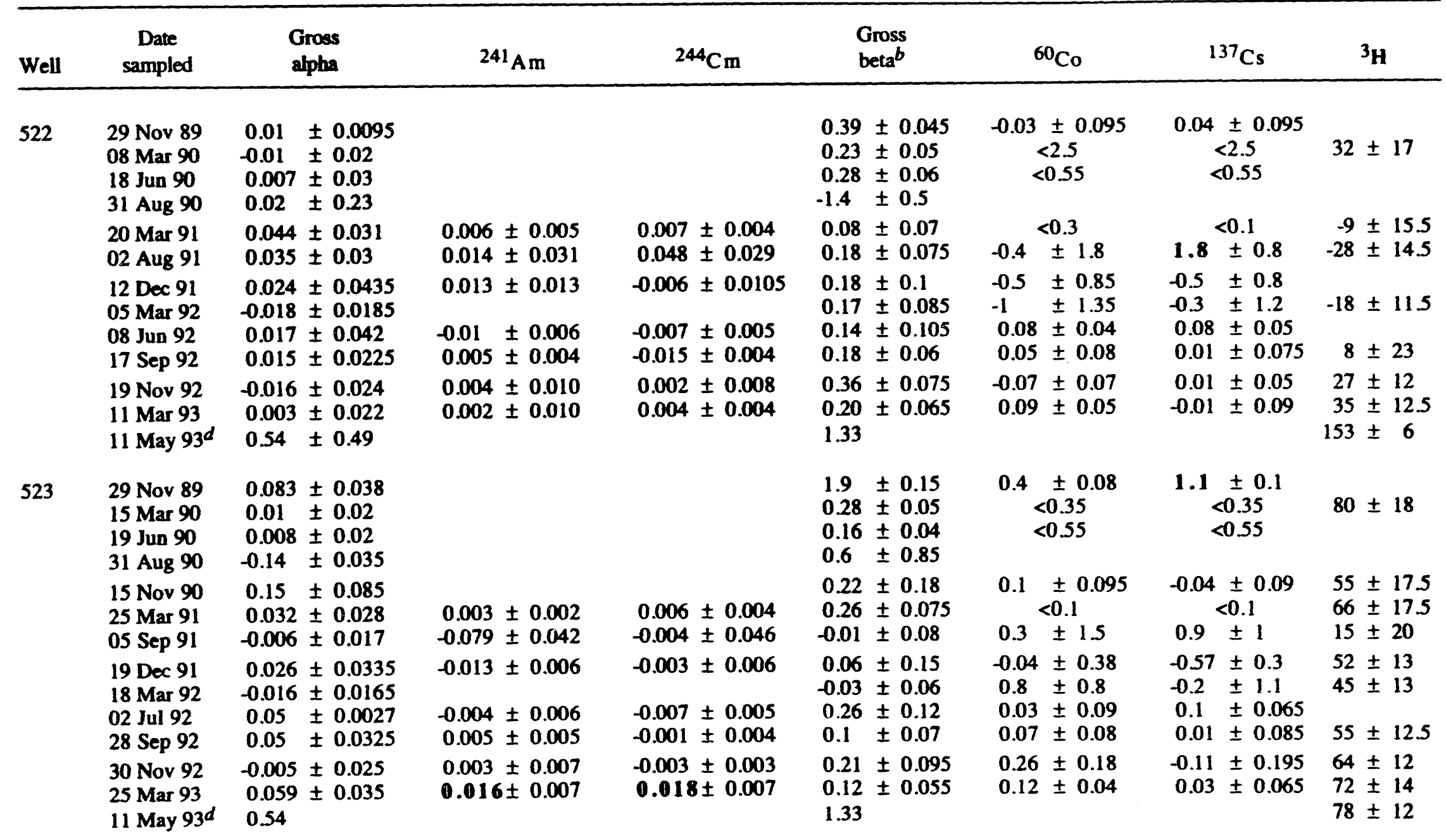


Table B.1 (continued)

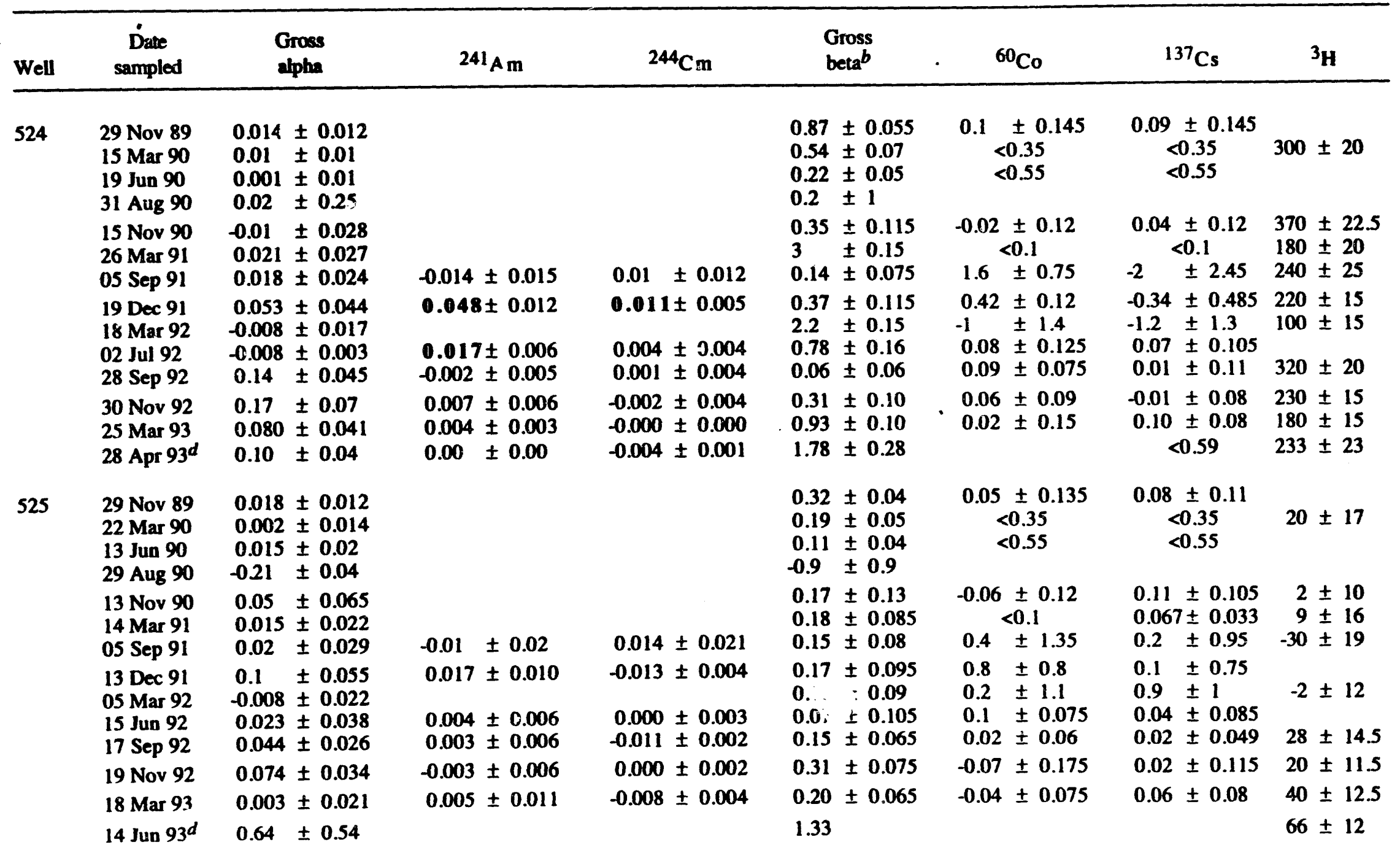


Table B.1 (continued)

\begin{tabular}{|c|c|c|c|c|c|c|c|c|}
\hline Well & $\begin{array}{c}\text { Dare } \\
\text { sampled }\end{array}$ & $\begin{array}{l}\text { Gross } \\
\text { alpha }\end{array}$ & 241 A m & ${ }^{244} \mathrm{Cm}$ & $\begin{array}{l}\text { Gross } \\
\text { beta }^{b}\end{array}$ & ${ }^{60} \mathrm{Co}$ & ${ }^{137} \mathrm{Cs}$ & ${ }^{3} \mathbf{H}$ \\
\hline 708 & $\begin{array}{l}06 \text { Dec } 90 \\
22 \text { Mar } 91 \\
04 \text { Sep } 91\end{array}$ & $\begin{array}{c}0.01 \pm 0.02 \\
0.022 \pm 0.021 \\
-0.043 \pm 0.026\end{array}$ & $\begin{array}{r}-0.002 \pm 0.003 \\
0.005 \pm 0.003\end{array}$ & $\begin{array}{ll}-0.002 & \pm 0.003 \\
0 & \pm 0.05\end{array}$ & $\begin{array}{l}0.1 \pm 0.06 \\
0.26 \pm 0.07 \\
0.11 \pm 0.09\end{array}$ & $\begin{array}{c}0.07 \pm 0.105 \\
<0.1 \\
0.7 \pm 1.05\end{array}$ & $\begin{array}{c}0.15 \pm 0.06 \\
<0.1 \\
0.89 \pm 0.415\end{array}$ & $\begin{aligned} 220 & \pm 20 \\
120 & \pm 20 \\
-6 & \pm 19.5\end{aligned}$ \\
\hline & $\begin{array}{l}16 \text { Dec } 91 \\
11 \text { Mar } 92 \\
24 \text { Jun } 92 \\
22 \text { Sep } 92\end{array}$ & $\begin{array}{c}0.005 \pm 0.0355 \\
-0.014 \pm 0.0035 \\
0.044 \pm 0.026 \\
0.081 \pm 0.0375\end{array}$ & $\begin{array}{l}0.01 \pm 0.006 \\
0.012 \pm 0.009 \\
0.004 \pm 0.004\end{array}$ & $\begin{array}{r}0.15 \pm 0.015 \\
0.075 \pm 0.013 \\
-0.006 \pm 0.004\end{array}$ & $\begin{array}{l}0.26 \pm 0.115 \\
0.01 \pm 0.07 \\
0.15 \pm 0.06 \\
0.17 \pm 0.08\end{array}$ & $\begin{array}{l}0.09 \pm 0.38 \\
0.1 \pm 0.8 \\
-0.01 \pm 0.105 \\
0.1 \pm 0.065\end{array}$ & $\begin{array}{l}0.15 \pm 0.35 \\
0.2 \pm 0.105 \\
0.03 \pm 0.08 \\
0.081 \pm 0.038\end{array}$ & $56 \pm 13.5$ \\
\hline & $\begin{array}{l}19 \text { Nov } 92 \\
18 \text { Mar } 93 \\
04 \text { Jun } 93^{d}\end{array}$ & $\begin{array}{l}0.22 \pm 0.08 \\
0.022 \pm 0.028 \\
0.53\end{array}$ & $\begin{array}{l}0.005 \pm 0.005 \\
0.007 \pm 0.004\end{array}$ & $\begin{array}{l}0.026 \pm 0.006 \\
-0.000 \pm 0.000\end{array}$ & $\begin{array}{l}0.42 \pm 0.115 \\
0.17 \pm 0.065 \\
1.96 \pm 1.03\end{array}$ & $\begin{array}{c}0.14 \pm 0.10 \\
-0.02 \pm 0.075\end{array}$ & $\begin{array}{r}0.02 \pm 0.095 \\
-0.07 \pm 0.065\end{array}$ & $\begin{array}{l}85 \pm 12.5 \\
93 \pm 14 \\
30\end{array}$ \\
\hline \multirow[t]{3}{*}{715} & $\begin{array}{l}15 \text { Nov } 90 \\
15 \text { Mar } 91 \\
29 \text { Aug } 91\end{array}$ & $\begin{array}{l}0.026 \pm 0.034 \\
0.076 \pm 0.039 \\
0.029 \pm 0.034\end{array}$ & $\begin{array}{l}0.008 \pm 0.009 \\
0.002 \pm 0.002\end{array}$ & $\begin{array}{r}-0.004 \pm 0.006 \\
0.004 \pm 0.003\end{array}$ & $\begin{array}{l}0.6 \pm 0.12 \\
0.34 \pm 0.075 \\
0.19 \pm 0.075\end{array}$ & $\begin{array}{c}-0.09 \pm 0.17 \\
<0.1 \\
-2 \quad \pm 2.4\end{array}$ & $\begin{array}{c}-0.03 \pm 0.14 \\
<0.1 \\
-0.8 \pm 2.45\end{array}$ & $\begin{array}{l}10 \pm 16.5 \\
42 \pm 16.5 \\
21 \pm 20\end{array}$ \\
\hline & $\begin{array}{l}10 \text { Dec } 91 \\
27 \text { Feb } 92 \\
01 \text { Jun } 92 \\
15 \text { Sep } 92\end{array}$ & $\begin{array}{ll}0.024 & \pm 0.031 \\
0 & \pm 0.020 \\
0.038 & \pm 0.027 \\
0.1 & \pm 0.04\end{array}$ & $\begin{array}{r}0.003 \pm 0.011 \\
0.006 \pm 0.014 \\
-0.008 \pm 0.006\end{array}$ & $\begin{array}{c}0.003 \pm 0.011 \\
0.005 \pm 0.005 \\
-0.008 \pm 0.0048\end{array}$ & $\begin{array}{l}0.37 \pm 0.11 \\
0.17 \pm 0.075 \\
0.16 \pm 0.065 \\
0.32 \pm 0.075\end{array}$ & $\begin{array}{ll}0.4 & \pm 0.8 \\
-0.7 & \pm 1.2 \\
0.17 & \pm 0.105 \\
0.01 & \pm 0.07\end{array}$ & $\begin{array}{ll}0.2 & \pm 0.8 \\
0.6 & \pm 1 \\
-0.17 & \pm 0.145 \\
0.05 & \pm 0.06\end{array}$ & $\begin{array}{l}26 \pm 12.5 \\
80 \pm 13.5\end{array}$ \\
\hline & $\begin{array}{l}17 \text { Nov } 92 \\
11 \text { Mar } 93 \\
24 \text { May } 93^{d}\end{array}$ & $\begin{array}{l}0.098 \pm 0.038 \\
0.042 \pm 0.035 \\
0.54\end{array}$ & $\begin{array}{r}0.013 \pm 0.008 \\
-0.039 \pm 0.014\end{array}$ & $\begin{array}{r}0.018 \pm 0.009 \\
-0.007 \pm 0.003\end{array}$ & $\begin{array}{l}0.34 \pm 0.07 \\
1.3 \pm 0.1 \\
1.33\end{array}$ & $\begin{aligned}-0.05 & \pm 0.11 \\
0.17 & \pm 0.12\end{aligned}$ & $\begin{array}{c}-0.01 \pm 0.105 \\
0.12 \pm 0.14\end{array}$ & $\begin{array}{l}67 \pm 12.5 \\
67 \pm 13.5 \\
86 \pm 14\end{array}$ \\
\hline
\end{tabular}


Table B.1 (continued)

\begin{tabular}{|c|c|c|c|c|c|c|c|c|}
\hline Well & $\begin{array}{c}\text { Date } \\
\text { sampled }\end{array}$ & $\begin{array}{l}\text { Gross } \\
\text { alpha }\end{array}$ & ${ }^{241} \mathrm{Am}$ & ${ }^{244} \mathrm{Cm}$ & $\begin{array}{l}\text { Gross } \\
\text { beta }^{b}\end{array}$ & ${ }^{60} \mathrm{Co}$ & ${ }^{137} \mathrm{Cs}$ & ${ }^{3} \mathbf{H}$ \\
\hline \multirow[t]{3}{*}{716} & $\begin{array}{l}06 \text { Dec } 90 \\
22 \text { Mar } 91 \\
04 \text { Sep } 91\end{array}$ & $\begin{array}{l}0.04 \pm 0.03 \\
0.026 \pm 0.03 \\
0.15 \pm 0.06\end{array}$ & $0.067 \pm 0.015$ & $0.039 \pm 0.012$ & $\begin{array}{l}0.27 \pm 0.08 \\
0.059 \pm 0.0095 \\
0.83 \pm 0.115\end{array}$ & $\begin{array}{c}0.11 \pm 0.095 \\
<0.1 \\
0.8 \pm 0.65\end{array}$ & $\begin{array}{c}0.09 \pm 0.1 \\
<0.1 \\
0.2 \pm 1.35\end{array}$ & $\begin{array}{l}-30 \pm 17 \\
-20 \pm 15.5 \\
-70 \pm 37\end{array}$ \\
\hline & $\begin{array}{l}16 \text { Dec } 91 \\
09 \text { Mar } 92 \\
24 \text { Jun } 92 \\
22 \text { Sep } 92\end{array}$ & $\begin{array}{ll}0.52 & \pm 0.36 \\
0.085 & \pm 0.0485 \\
0.09 & \pm 0.065 \\
0.03 & \pm 0.0275\end{array}$ & $\begin{array}{l}0.01 \pm 0.007 \\
0.007 \pm 0.006 \\
0.007 \pm 0.008\end{array}$ & $\begin{array}{l}0.022 \pm 0.005 \\
0.002 \pm 0.004 \\
0.003 \pm 0.006\end{array}$ & $\begin{array}{l}1.1 \pm 0.3 \\
0.66 \pm 0.11 \\
0.28 \pm 0.115 \\
0.08 \pm 0.055\end{array}$ & $\begin{array}{ll}0.5 & \pm 0.7 \\
1.6 & \pm 0.9 \\
0.23 & \pm 0.05 \\
0.51 & \pm 0.08\end{array}$ & $\begin{array}{l}-0.2 \pm 0.8 \\
0.1 \pm 1.1 \\
0.04 \pm 0.12 \\
0.8 \pm 0.075\end{array}$ & $-5 \pm 10.5$ \\
\hline & $\begin{array}{l}22 \text { Sep } 92 \\
25 \text { Nov } 92 \\
09 \text { Mar } 93\end{array}$ & $\begin{array}{l}0.09 \pm 0.05 \\
0.003 \pm 0.021\end{array}$ & $\begin{array}{l}0.007 \pm 0.007 \\
0.004 \pm 0.003\end{array}$ & $\begin{array}{r}0.014 \pm 0.007 \\
-0.003 \pm 0.004\end{array}$ & $\begin{array}{l}0.60 \pm 0.075 \\
0.21 \pm 0.06 \\
0.20 \pm 0.03\end{array}$ & $\begin{array}{l}0.01 \pm 0.11 \\
0.01 \pm 0.065\end{array}$ & $\begin{array}{c}-0.09 \pm 0.05 \\
0.048 \pm 0.049 \\
<0.74\end{array}$ & $\begin{array}{l}35 \pm 12.5 \\
92 \pm 14 \\
15 \pm 9\end{array}$ \\
\hline
\end{tabular}

a SWSA = Solid Waste Storage Area. Concentrations are reported in Bq/ \pm 1 SE (counting error). Boldface indicates analysis was not performed.

or is significantly $>0$ (t-test, $\alpha=0.05$ ) based on

$b$ Gross beta analysis does not include ${ }^{3} \mathrm{H}$.

I Waste Area Grouping 5. Counting error not reported by Bechtel for all

$d$ Data provided by Bechtel National, Inc., as part of
analyses. Only data from filtered samples are reported.

e Duplicate sample.

$f$ Second value reported for same sample. 
Table B.2. White Oak Creek bank seep and tributary monitoring results from SWSA 5 North ${ }^{a}$

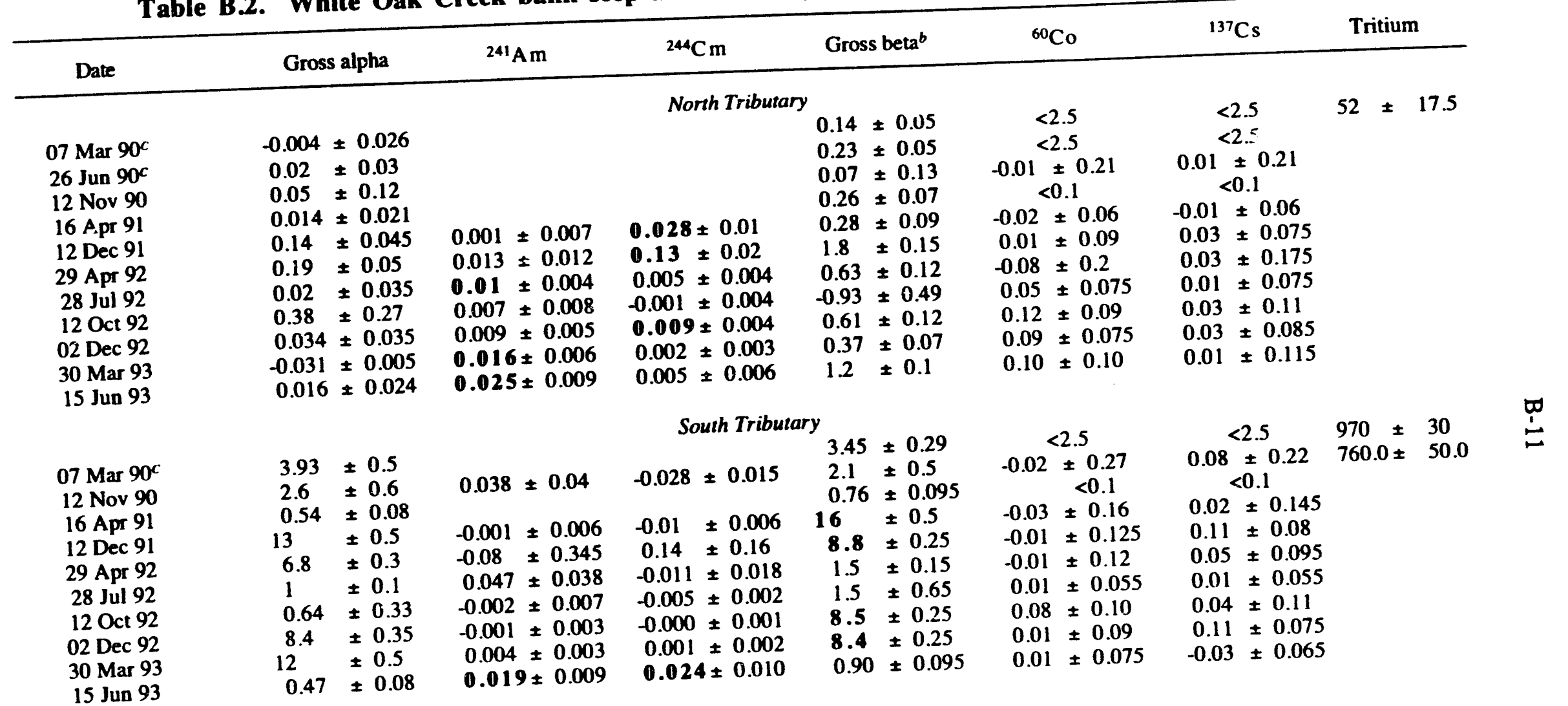


Table B.2. continued

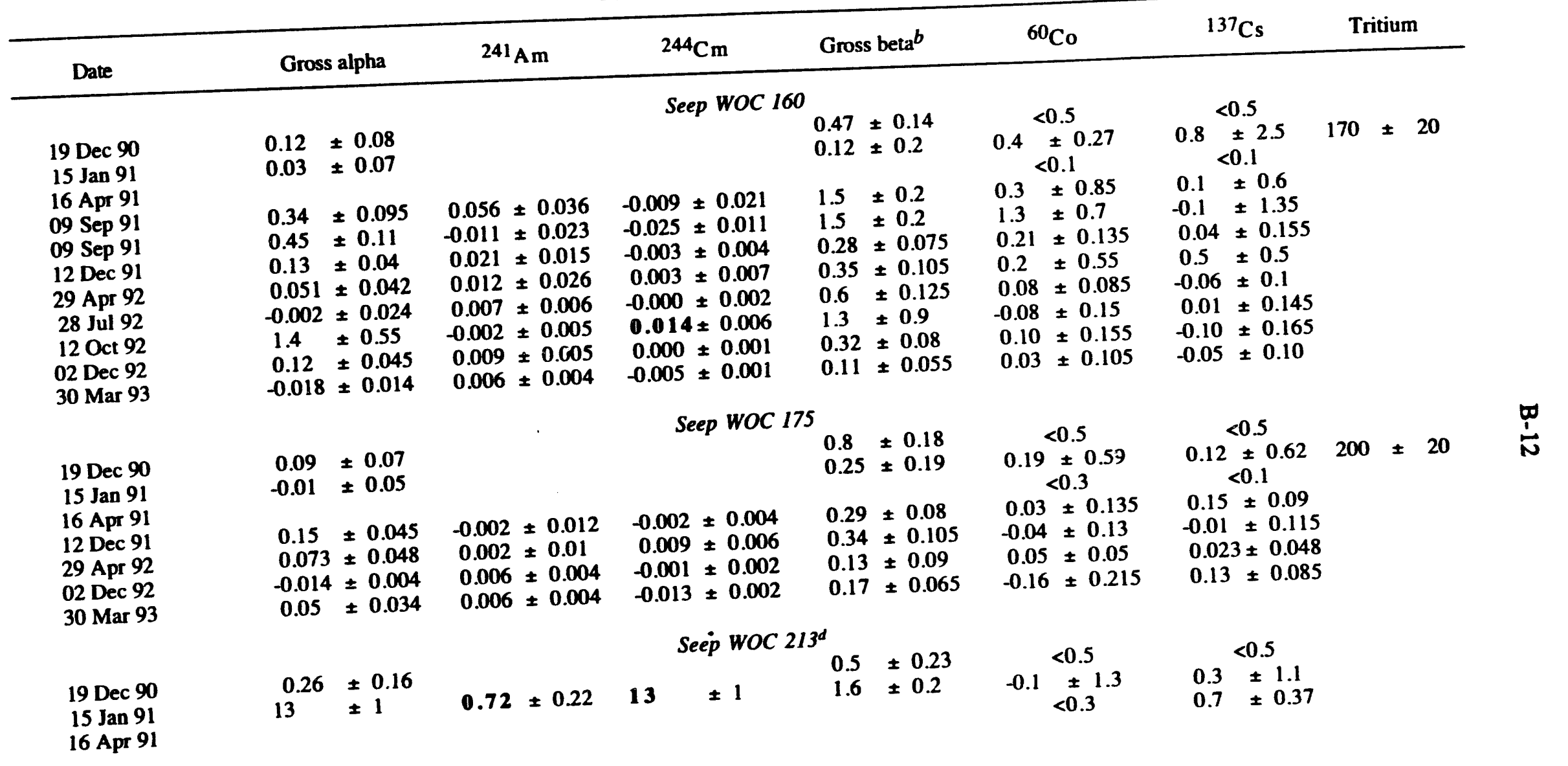


Table B.2. continued

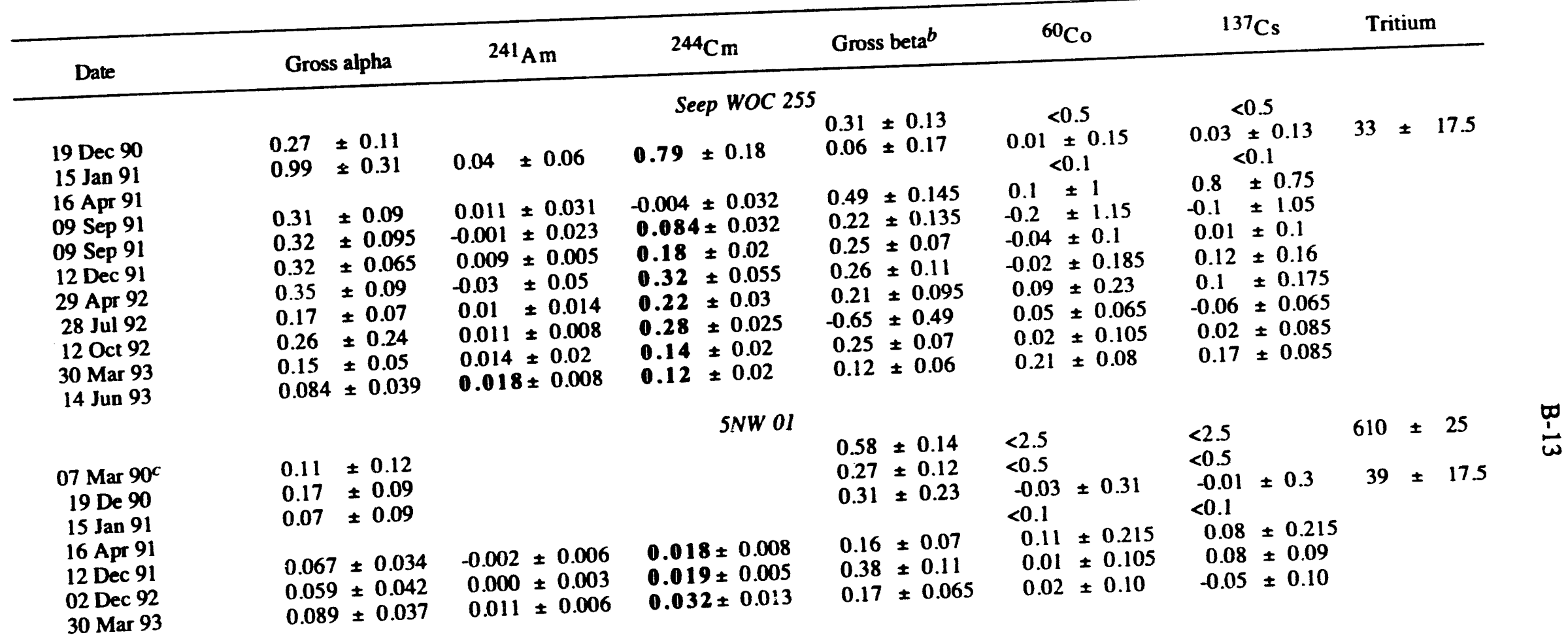


Table B.2. continued

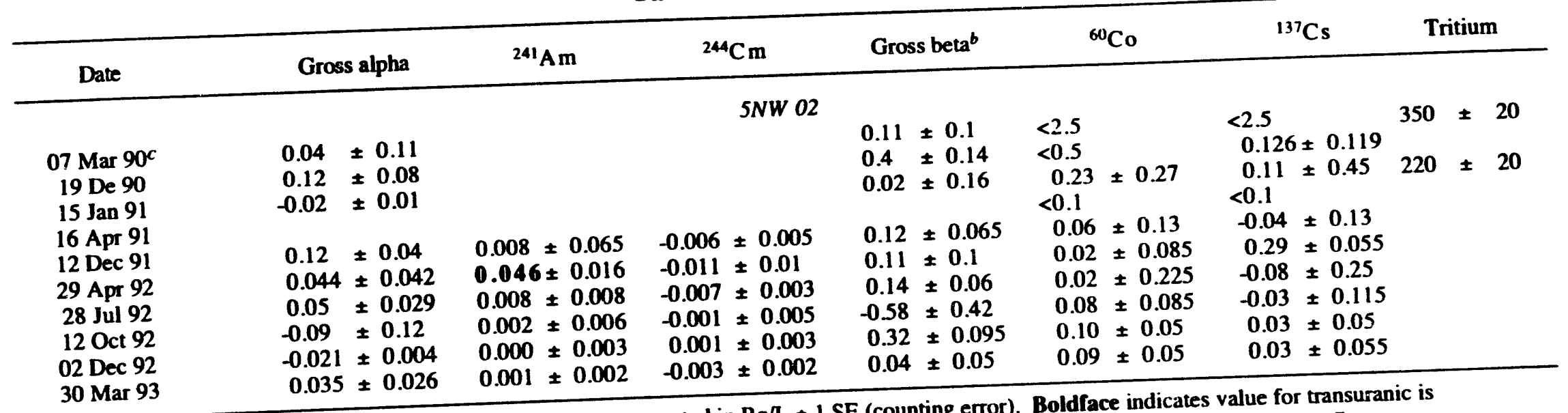

a SWSA = Solid Waste Storage Area. Concentrations are reported in BqL \pm 1 SE (counting error). Boldioce inds because of insufficient flow.

$b$ Gross beta does not include tritium.
$c$ Gross alpha/beta analyses for these samples were performed in the Health and Safety Research Division following sample preparation in the

$c$ Gross alpha/beta analyses for these samples were performed in the Health amd lower than values from analyses performed in Analytical Chemistry Division.

Environmental Sciences Division (ESD).

Gamma scans were performed in the ESD counting room.
$d$ Seep WOC 213 was not sampled after April 1991 because of insufficient flow. 
Table B.3. Radiological data from well 516 geochemical investigation ${ }^{a}$

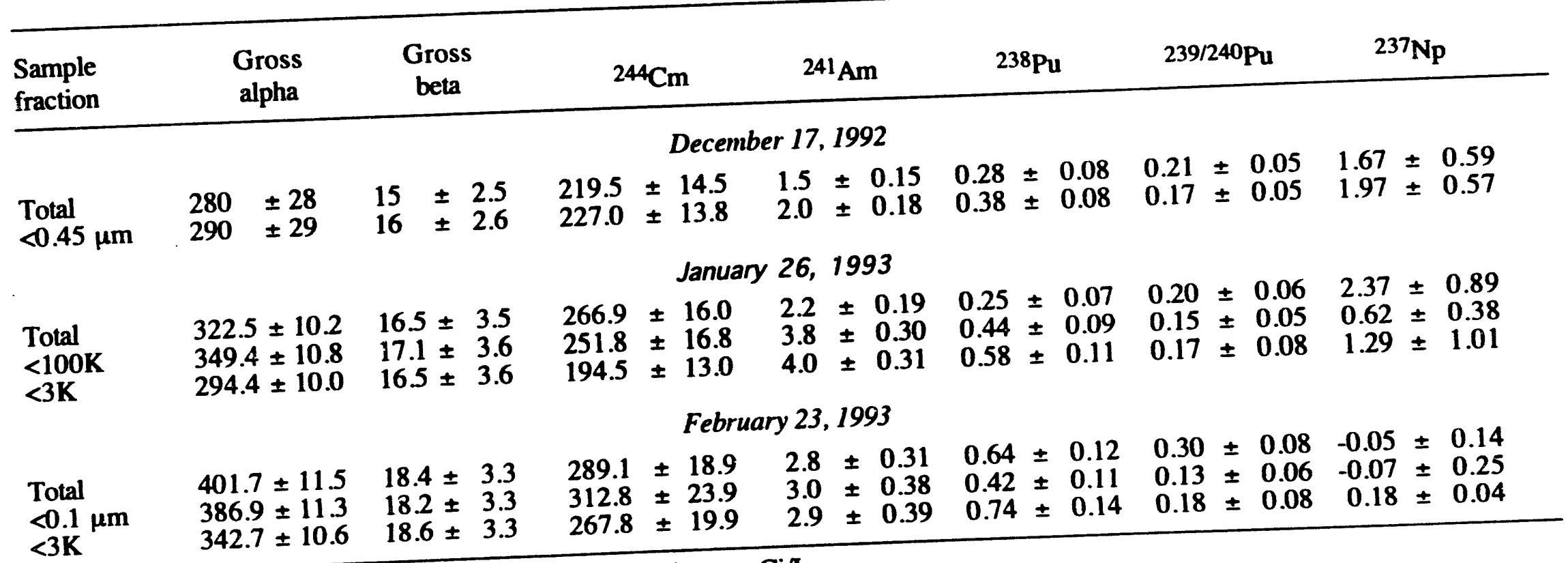

a All values are listed as mean \pm counting error. Units are $\mathrm{pCi} / \mathrm{L}$.

$b$ Total = unfiltered sample; $<0.45 \mu \mathrm{m}=$ portion of sample passing a $0.45-\mu \mathrm{m}$ sample passing a $0.1-\mu \mathrm{m}$ ultra filter.

ilter; $<3 \mathrm{~K}=$ portion of sample passing a $3 \mathrm{~K}$ ultrafilter; $<0.1 \mu \mathrm{m}=$ portion of sample passing a $0.1-\mu \mathrm{m}$ ultra 
Table B.4. Major cations and trace metals in well 516 geochemical investigation ${ }^{a}$

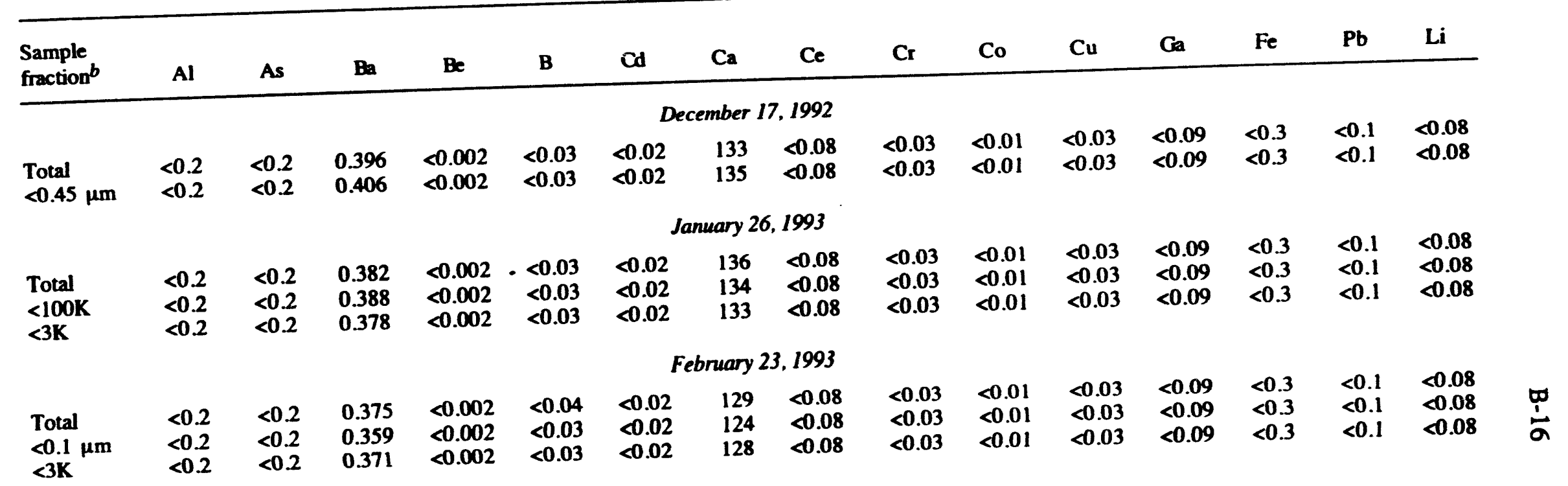


Table B.4 cont.

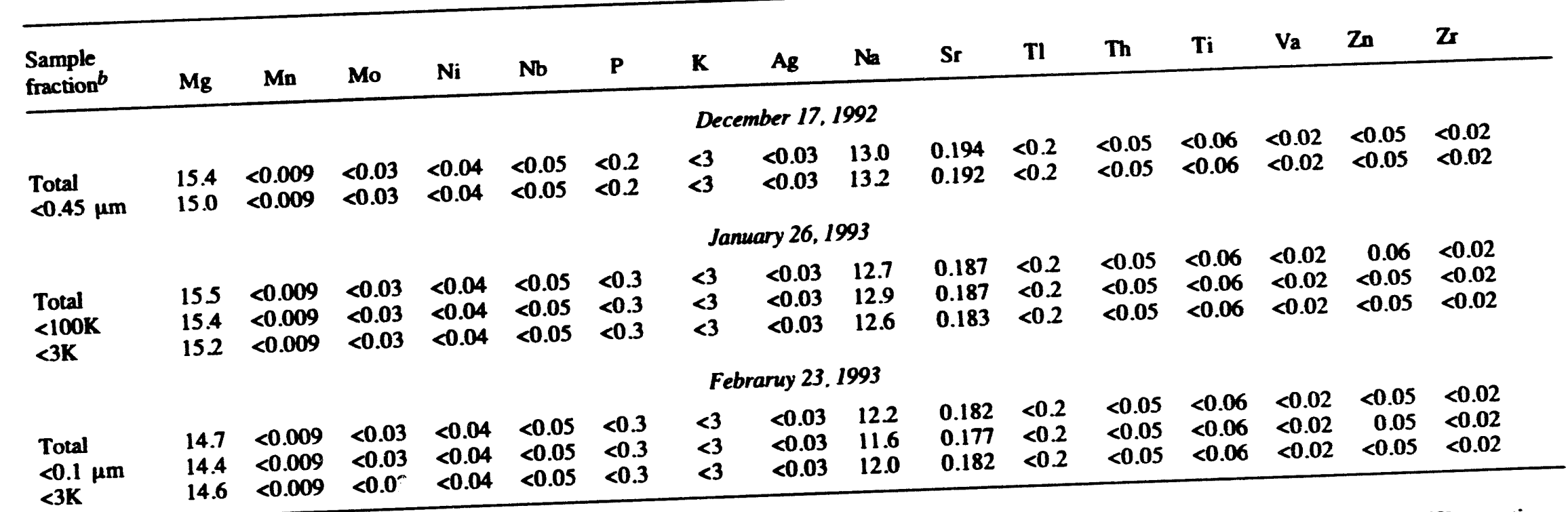

$a$ Units are $\mathrm{mg} / \mathrm{L}$.

b Units are mglt.
Total $=$ unfiltered sample; $<0.45 \mu \mathrm{m}=$ portion of sample passing a $0.45-\mu \mathrm{m}$ filter, $<100$
of sample passing a $3 \mathrm{~K}$ ultrafilter; $<0.1 \mu \mathrm{m}=$ potion of sample passing a $0.1-\mu \mathrm{m}$ ultrafilter. 
Table 5. Anions and water chemistry data from well 516 geochemistry investigation

\begin{tabular}{|c|c|c|c|c|c|c|c|c|c|c|c|c|c|c|c|c|}
\hline $\begin{array}{l}\text { Sample } \\
\text { Fraction } b\end{array}$ & $\begin{array}{c}\mathrm{Br} \\
(\mathrm{mg} / \mathrm{L})\end{array}$ & $(\mathrm{mg} / \mathrm{L})$ & $\underset{(m g / L)}{F}$ & $\begin{array}{l}\text { Nitrate } \\
\text { (as N) } \\
\text { (mg/L) }\end{array}$ & $\begin{array}{c}\text { Nitrite } \\
\text { (as N) } \\
\text { (mg/L) }\end{array}$ & $\begin{array}{c}\text { Total } \\
\mathrm{PO}_{4} \\
(\mathrm{mg} / \mathrm{L})\end{array}$ & $\begin{array}{c}\mathrm{SO}_{4} \\
(\mathrm{mg} / \mathrm{L})\end{array}$ & $\begin{array}{l}\text { Total } \\
\text { alkalinity } \\
\text { (mgl) }\end{array}$ & $\begin{array}{l}\text { Organic } \\
\text { carbon } \\
\text { (mg/L) }\end{array}$ & pH & $\underset{(m V)}{\text { dH }}$ & $\begin{array}{l}\text { Dissolved } \\
\text { oxygen } \\
\text { (ppm) }\end{array}$ & $\begin{array}{c}\text { Specific } \\
\text { conductance } \\
(\mu \mathrm{mhos} / \mathrm{cm})\end{array}$ & $\begin{array}{l}\text { Temp. } \\
\text { ('C) }\end{array}$ & $\begin{array}{l}\text { Filterable } \\
\text { Residue } \\
\text { (mg/L) }\end{array}$ & $\begin{array}{c}\text { Turbidity } \\
\text { (NIU) }\end{array}$ \\
\hline \multicolumn{17}{|c|}{$\begin{array}{llll}1.290 & 3.80 & <0.25 & <0.625 \\
0.511 & 3.86 & <0.25 & <0.625 \\
\end{array}$} \\
\hline
\end{tabular}

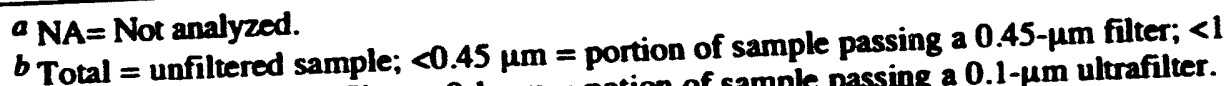

b Total = unfiltered sample; $<0.45 \mu \mathrm{m}=$ portion of sample passing a $0.1-\mu \mathrm{m}$ ulturailter.
of sample passing a $3 \mathrm{~K}$ ultrafilter; $<0.1 \mu \mathrm{m}=$ potion of sample 
Table B.6. Ratios of ${ }^{240} \mathrm{Pu}$ to ${ }^{244} \mathrm{Cm}$ and calculated migration times for well 516 in SWSA 5 Northa

\begin{tabular}{cccc}
\hline${ }^{244} \mathrm{Cm}(\mathrm{pCi} / \mathrm{L})$ & $240 \mathrm{Pu}(\mathrm{pCi} / \mathrm{L})$ & Ratio $(\mathrm{Pu} / \mathrm{Cm})$ & Time (years) \\
\hline 219.5 & 0.21 & $9.567 \mathrm{E}-04$ & 7.777 \\
266.9 & 0.20 & $7.493 \mathrm{E}-04$ & 6.276 \\
289.1 & 0.30 & $1.038 \mathrm{E}-03$ & 8.342 \\
\hline
\end{tabular}

a SWSA = Solid Waste Storage Area 


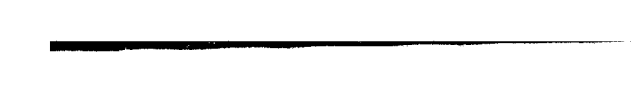




\section{APPENDIX C RELEVANT CORRESPONDENCE}

C-1 


\section{Department of Energy}

Oak Ridge Field Office

P.O. Box 2001

Oak Ridge, Tennessee 37831-

$$
\text { April 9, } 1993
$$

Mr. Charlie E Frye, Director

Energy Systems Waste Management Organization

Martin Marietta Energy Systems, Inc.

Post Office Box 2003

Oak Ridge, Tennessee 37831-7357

Dear Mr. Frye:

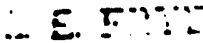

NPR 141993

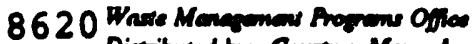

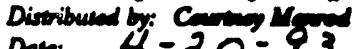

H.L Ader T. R. Scantar

D. B. Buchañon C. B. Socon

A. D. Chy - B. V. Shetion

E. C Gon

V. K Evers

R C Macoun

LE. MeNem

$R$ C Stomin

LE Simarian

M. W. Tut

D. W. Tumar

\section{Due Dave}

Cops/Rouletione

Fib SWUSA 50

\section{STORAGE OF TRANSURAVIC WASTE AT SOID WASTE STORAGE AREA 5} NORTH

This is in response to your letter dated December 10, 1992, regarding the concerns with transuranic (TRU) waste storage at Los Aamos National Laboratory (LANL). The LANL situatien has caused the U.S. Department of Energy Waste Management and Technology Development Division (WM \& TD) to focus on the status of TRU wastes stored at Oak Ridge National Laboratory's Solid Waste Storage Area 5 North (SWSA SN). As a result, Harvey Rice of my staff hes conducted a preliminary investigation of this issue; his findings are presented in Enclosure 1.

In brief, WM \& TD is concerned about the Martin Marietta Energy Systems, Inc, followup on the apparent "release" of IRU constituents at SWSA SN. Although appropriate response/monitoring actiom appear to have been taken, the overall tracking of the situation seems somewhat fragmented and not fully documented which could present difficulties in defending our action. By way of this letter, I am requesting that your division review our findings and provide me with an update no later than May 30, 1993.

If you have any question, please contact Harvey Rice (Program Manager) at 241-2157.

Sincerely,

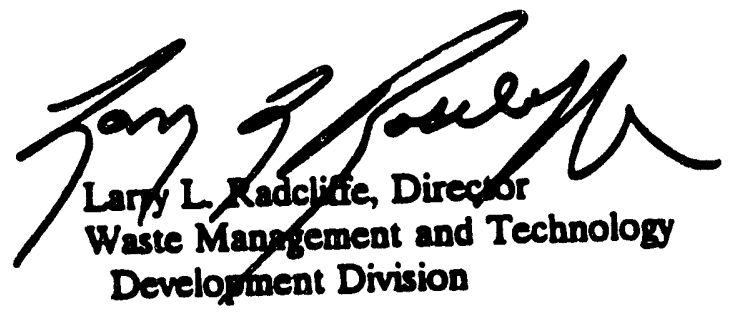

Enclosure

ce: Cowne us Carmerr $x$

cc w/enclosure:

M. Roddyc, EW-922

J. Nelson, PAI

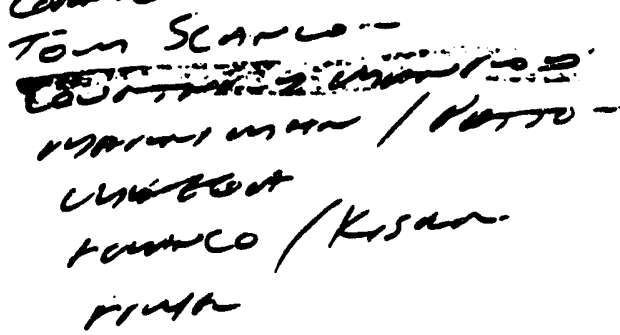

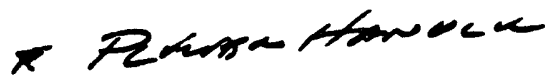


Chronology of Events:

10/70-09/79 TRU and TRU mixed wastes were stored in trenches in SWSA SN.

02/14/90 Issuance of January 1990 Monthly Report for the Active Sites Environmental Monitoring Program (ASEMP) marks the initiation of this program. Well 516 sampled on November 29, 1989, showed gross alpha activity and Curium-244 (24 Cm) of 170 and $140 \mathrm{Bg} / \mathrm{L}$, respectively. The report states: "The first full sampling of wells, streams and stream sediments in and around SWSA 5 North is planned for February. If results ... confirm the previous results, an action plan for further investigation will be developed."

03/12/90 Issuance of February 1990 ASEMP Monthly Report; delay of first full sampling until March'.

04/16/90 Issuance of March 1990 ASEMP Monthly Report'.

05/07/90 Issuance of April 1990 ASEMP Monthly Report.

06/07/90 Issuance of May 1990 ASEMP Monthly Report. Gross alpha activities of March 7, 1990, sediment and stream samples range from 200 to $1600 \mathrm{Bg} / \mathrm{kg}^{2}$. A site map showing all well and stream/seep sampling locations in SWSA SN is contained in this report, and provided as Figure $I$ of this attachment.

07/09/90 Issuance of June 1990 ASEMP Monthly Report. Well 516 sample taben in March 1990 showed gross alpha activity, Americium-241 ( $\left.{ }^{21} \mathrm{Am}\right)$, and ${ }^{24} \mathrm{Cm}$ of $79,5.5$, and $71 \mathrm{Bg} / \mathrm{L}$, respectively.

08/07/90 Issuance of July 1990 ASEMP Monthly Report. Well 516 sample taken on June 26, 1990, shows gross alpha activity of $9.3 \mathrm{Bg} / \mathrm{L}^{3}$.

09/07/90 Issuance of August 1990 ASEMP Monthly Report. Table 11 of the Report documents results from splits of the June 26, 1990, sample. Other labs reported gross alphn activities of 120 and $129.5 \mathrm{~Bq} / \mathrm{L}^{3}$. Results are also presented for an August 16, 1990, sample which was both "pre-" and "post-acidified". Gross alpha activities are shown in Table $I$ below.

Table 1. Gross Alpha Activity on August 16.1990

\begin{tabular}{lrr} 
Type of Sample & ESD & ACD \\
\hline Pre-acidified & 18.44 & 110 \\
Post-acidified & 20.89 & 85 \\
\hline
\end{tabular}

ESD = Environmental Sciences Division

$A C D=$ Analytical Chemistry Division 


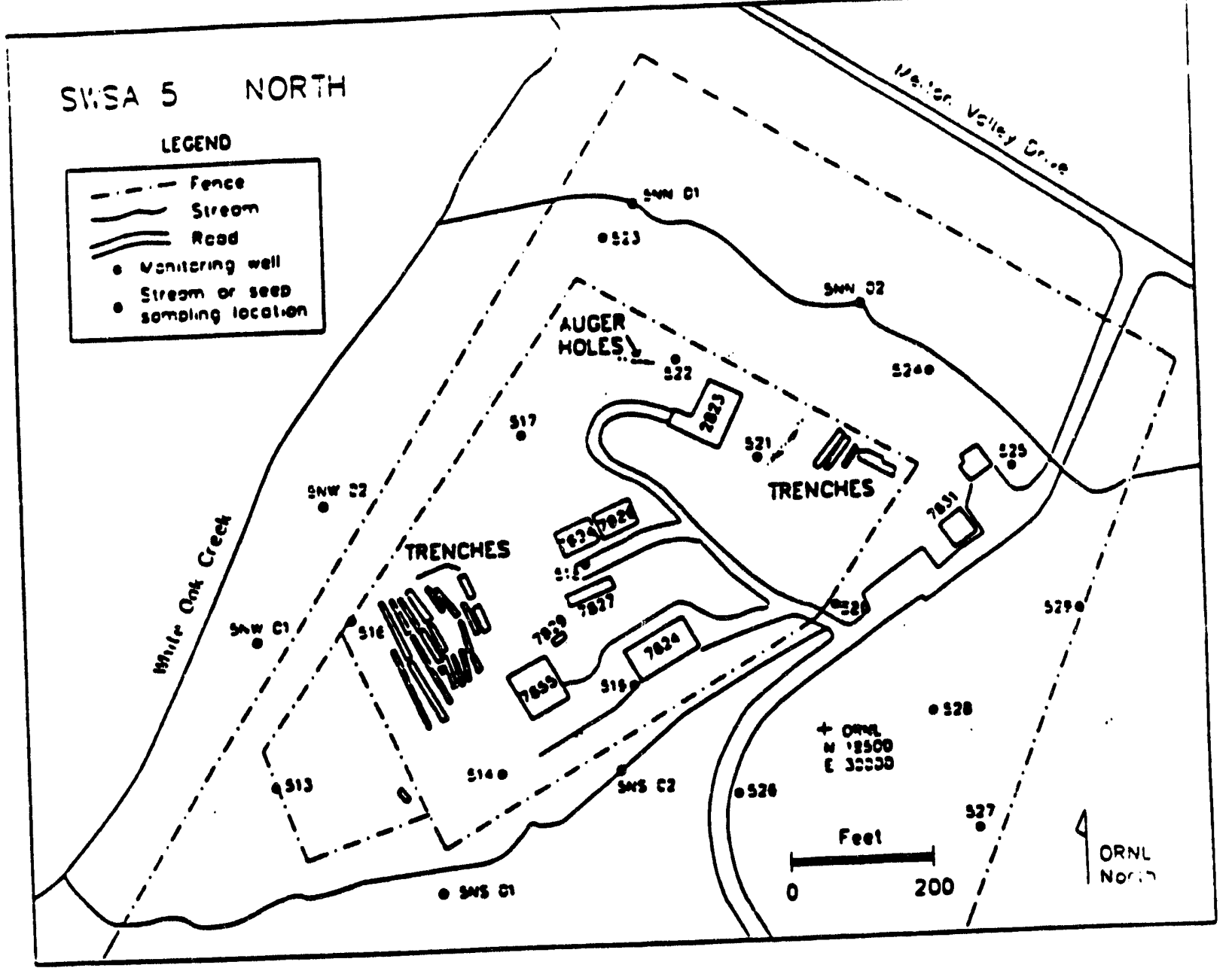

Figure 1. Monitoring Wells and Stream and Seep Sampling Locations in and around SWSA SN 


\section{$c-6$ \\ ATTACBMIDNT 1 \\ INVSTIGATION OF TRU WASTE STORAGE AT SWSA SN (continued)}

Chronology of Erents (Continued):

The August report also states that: "It now appears that gross alpha ... analyses performed in ESD ... were low by as much as a factor of $10^{\circ}$ when compared to results from both ACD and the Y-12 lab. "This evidence (that most of the alpha activity is from dissolved material) supports the suggestion that the alpha activity is from wastes emplaced in the trenches upgradient from the well."

10/08/90 Issuance of September 1990 ASEMP Monthly Report.

10/19/90 Submittal of Work Plan for Further lmvestigation of Possible TRU Contanination in SWSA 5 North by Tom Ashwood (ESD).

11/07/90 Issuance of October 1990 ASEMP Monthly Report. Sediment sample taken on September 13, 1990, from the North Tributary shows alpha activity of 380 $\mathrm{Bq} / \mathrm{kg}^{2}$.

12/06/90 Issuance of November 1990 ASEMP Monthly Report.

01/04/91 Issuance of December 1990 ASEMP Monthly Report. Table 12 of the Report documents results of a December 1990 sampling. Of Well 516 when gross alpha activity was $38 \mathrm{Bg} / \mathrm{L}$. A summary of both-groundwater and surface water analyses follows in Table 2 below (All analyses are in $\mathrm{Bq} / \mathrm{L}$ ).

Table 2. Analysen of Well 516 (December 1990)

\begin{tabular}{lcccc}
$\begin{array}{c}\text { Sample } \\
\text { Location }\end{array}$ & $\begin{array}{c}\text { Gross } \\
\text { Alpha }\end{array}$ & ${ }^{m} \mathrm{Cm}$ & ${ }^{21} \mathrm{Am}$ & ${ }^{2 \times \mathrm{Pu}}$ \\
\hline Well 516 & 38 & 41 & 1.7 & 0.11 \\
SNST 20 $^{\circ}$ & 2.6 & $-0.03^{\circ}$ & 0.04 & 0.01 \\
Mouth $^{\circ}$ & 2.8 & 0.06 & $-0.01^{\circ}$ & 0.005 \\
\hline
\end{tabular}

See Figure 2 for locations of surface-water samples.

Value is not significantly greater than 0.00 ( $p<0.01$ ).

02/05/91 Iamance of January 1991 ASEMP Monthly Report. 
Chronology of Events (Continued):

02/11/91 Submittal of Preliminary Resulss of Investigation into IRU Consamination in SWSA 5 North Groundwater by Tom Ashwood (ESD). Conclusions were:

1. Both ${ }^{2 m} \mathrm{Cm}$ and ${ }^{211} \mathrm{Am}$ are present in White Oak Creek (WOC) adjacent to SWSA SN.

2. The WOC transuranics may have another source besides the buried TRU waste in SWSA 5N.

3. Sediment in WOC may be a source of transuranics.

Recommendations were:

1. Investigate the source of ${ }^{2 m} \mathrm{Cm}$ and ${ }^{21} \mathrm{Am}$ in WOC4.

2. Resample Well 982 and analyze for TRU elements.

3. Add four new seep sites and Wells 708 and 715 to regular ASEMP quarterly sampling.

02/28/91 Issuance of Final Occurrence Report by H. L. Adair [Energy Systems Solid Waste Operations (SWO)]. This states that the "occurrence" was discovered on October 2, 1990, and that the cause of contamination may or may not be the TRU waste in SWSA SN trenches. Commitments we're:

1. Sample "small stream south of SWSA-S North" routinely.

2. Place Manganese Oxide (MnO) fiber pockets at various locations in the stream to concentrate the contaminants.

3. Sample seeps in the area.

02/91 Issuance of Active Sites Environmental Monitoring Program: Mid-FY 1990 Summary Report, ORNLM-1179.

03/05/91 Issuance of February 1991 ASEMP Monthly Report. The report states: "No evidence has been found to suggest that the burial trenches in SWSA 5 North are the source of ${ }^{21} \mathrm{Cm}$ and ${ }^{211} \mathrm{Am}$ in ... White Oak Creek ... The (TRU) activity is probably associated with sediment, and its source is unclear ... Elevated gross alpha activity in the tributary south of SWSA SN is not caused by (TRU) elements. No further investigation is planned (beyond quarterly sampling)."

04/05/91 Issuance of March 1991 ASEMP Monthly Report. A sample taken from WOC 213 Seep on March 14, 1991, showed gross alpha activity, ${ }^{211} \mathrm{Am}$, and ${ }^{24} \mathrm{Cm}$ levels of $13,0.72$, and $13 \mathrm{~Bq} / \mathrm{h}$, respectively.

05/06/91 Issuance of April 1991 ASEMP Monthly Report'.

06/06/91 Issuance of May 1991 ASEMP Monthly Report'. 
INVESTGATION OF TRU WASTE STORAGE AT SWSA SN (continued)

Chronology of Erents (Continued):

07/08/91 Issuance of June 1991 ASFMP Monthly Report.

08/05/91 Issuance of July 1991 ASEMP Monthly Report'.

09/06/91 Issuance of August 1991 ASEMP Monthly Repont.

10/07/91 Issuance of September 1991 ASEMP Monthly Report'.

10/91 Issuance of Active Sites Environmental Monitoring Program: Action Levels by J. S. Ashwood and T. L. Ashwood. Table 4 documents the following recommended action levels for SWSA SN: gross beta activity, $2.0 \mathrm{~Bq} / \mathrm{L}$; Cobalt-60 (10 Co), 1.5 Bq/L; and Cesium-137 ( $\left.{ }^{157} \mathrm{Cs}\right), 1.0 \mathrm{Bg} / \mathrm{L}$. The document states: "No action level is proposed for gross alpha in SWSA SN because ASEMP is moving away from gross alpha as a screening measurement in this area. The analyses for specific transuranics ... are more sensitive ... and can quantify the presence of transuranics at levels that would be undetectable by the gross alpha activity screen. ..."

10/05/913 Issuance of October 1991 ASEMP Monthly Report. Well 516 showed gross Upha activities of 210 and $46 \mathrm{~Bq} / \mathrm{L}$ in samples triken on March 22 and September 4, 1991, respectively. Various other wells (and two seep sites) showed measurable levels of ${ }^{241} \mathrm{Am}$ and/or ${ }^{24} \mathrm{Cm}$.

10/91 Issuance of Active Sites Environmental Monitoring Program: FY 1990 Report, ORNL/M-327.

10/91 Issuance of Active Sises Environmental Monitoring Program: Mid-FY 1991 Repor, ORNLM-1442.

12/05/91 Issuance of November 1991 ASEMP Monthly Report.

(undated) Issuance of December 1991 ASEMP Monthly Report'.

02/92 Issunce of Active Sites Environmental Monitoring Progran: Program Plan, Rev. I by T. L. Ashwood et al. Highlights of this document are:

1. Number of sampling locations were increased from those shown in Figure I to those shown in Figure 2.

2. Analytes for all groundwater and surface-water samples are: gross alpha activity, gross beta activity, gamma scan, ${ }^{21} \mathrm{Am}$, and ${ }^{24} \mathrm{Cm}$. In addition, groundwater is to be analyzed for tritium (Table B.5).

3. Table B.1 documents action levels identical to those in the Ashwood document cited 10/91. 


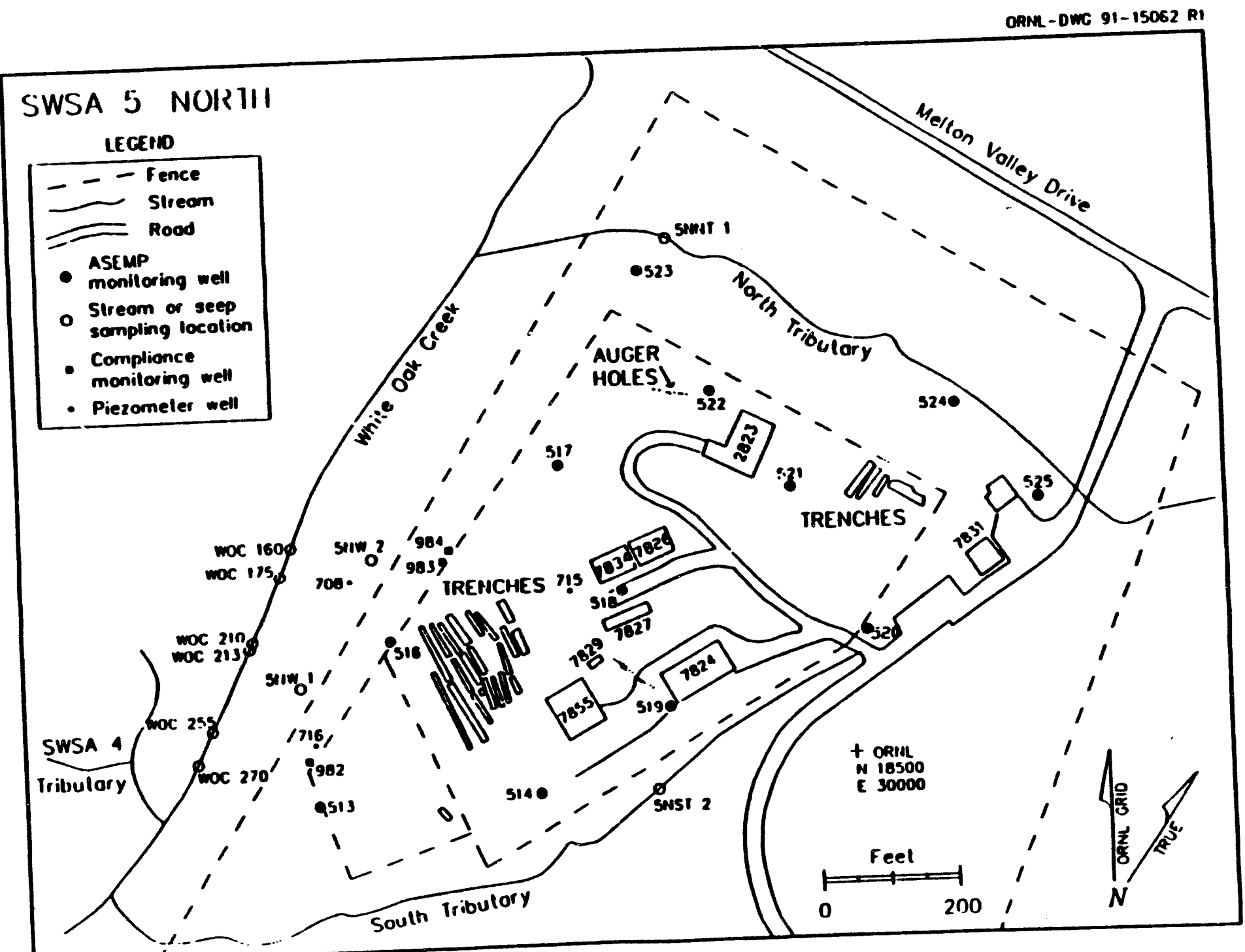

Figure 2. Transuranic Waste Storage Sites and Sampling Locations in SWSA 5N 


\section{$C-10$ \\ ATTACBMDTIT 1 \\ MVESTIGATION OF TRU WASTE STORAGE AT SWSA SN (continued)}

Chronology of Erents (Continued):

4. The following statement is made: "Samples that exceed an established action level or that otherwise indicate a problem will be reported...Depending upon the nature of the problem, an action plan may be prepared describing those actions to further investigate and/or remedy the problem..." (pp. 11 and 12, Section 4.3).

02/06/92 Iscuance of January 1992 ASEMP Monthly Report.

03/06/92 Issuance of February 1992 ASEMP Monthly Report.

04/06/92 Isounce of March 1992 ASEMP Monthly Report.

05/07/92 Issuance of April 1992 ASEMP Monthly Report. Well 516 sample taken on December 18, 1991, showed gross alpha activity of $24 \mathrm{Bg} / \mathrm{L}$. Various other wells showed measurable levels of ${ }^{211} \mathrm{Am}$ and/or ${ }^{21} \mathrm{Cm}$.

06/05/92 Isruance of May 1992 ASEMP Monthly Report.

07/08/92 Isuance of June 1992 ASEMP Monthly Report?

08/05/92 Iswance of July 1992 ASEMP Monthly Report.

09/09/92 Iswance of August 1992 ASEMP Monthly Report. Well 516 sample taken on June 30, 19:?, showed gross alpha activity, ${ }^{21} \mathrm{Am}$, and ${ }^{2 m} \mathrm{Cm}$ levels of $14,0.18$, and $15 \mathrm{Bg} / \mathrm{L}$, respectively. Various other wells, seeps, and stream samples showed measurable levels of gross alpha activity, ${ }^{24} \mathrm{Am}$, and/or ${ }^{24} \mathrm{Cm}$.

10/06/92 Iswance of September 1992 ASEMP Monthly Report.

11/06/92 Isuance of October 1992 ASEMP Monthly Report.

11/92 Irannce of Active Sites Environmental Monitoring Program: FY 1991 Report, O.NLM-1792.

12/07/92 Inunce of November 1992 ASEMP Monthly Report.

01/06/93 Iswance of December 1992 ASEMP Monthly Report. Analytical results are provided for samples taken September 16 to 28, 1992. Well 516 showed gross alpha activity, ${ }^{211} \mathrm{Am}$, and ${ }^{24} \mathrm{Cm}$ levels of $6.4,0.019$, and $0.36 \mathrm{~Bq} / \mathrm{L}$, rexpectively. Various other wells showed measurable levels of ${ }^{21} \mathrm{Am}$ and/or ${ }^{2 m} \mathrm{Cm}$. 


\section{$C-11$ \\ ATTACAMDNT 1 \\ INVESTIGATION OF TRU WASTE STORAGE AT SWSA SN (continued)}

\section{Chronology of Erents (Continued):}

01/22/93 ASEMP and ESD personnel gave verbal indication that, although action levels for ${ }^{21} \mathrm{Am}$ and ${ }^{24} \mathrm{Cm}$ do not exist per se, any statistically significant detection of these constituents is an action level. These levels are not "official," nor are they riskbased.

\section{Summary of Concerns:}

1. Although TRU wastes have been placed in SWSA 5N since October 1970, monitoring of groundwater and surface water did not begin until January 1990.

2. DOE-WMD is not clear as to the action levels (ALs) prior to the issuance of Rev. 1 of the Program Plan (i.e., February 1992). Gross alpha activity in excess of $1 \mathrm{Bg} / \mathrm{L}$ is the only Al which has come to light thus far, and this appears to be undocumented. Likewise, ALs for ${ }^{21} \mathrm{Am}$ and ${ }^{24} \mathrm{Cm}$ are said to be any statistically significant detection, but this too appears to be undocumented.

3. The AL for gross alpha activity was exceeded by factors of 170,79 , and 9 for samples taken from Well 516 on November 29, 1989, and March 28 and June 26, 1990, respectively. However, these appear to have gone unreported until the October 1990
Occurrence Report.

4. No analytical or sample-preparation methods are given in any of the documents reviewed. The concem about when to acidify samples before gross-alpha analyses might not have arisen if standard analytical procedures had been used.

5. In spite of numerous attempts by Energy Systems, DOE-OR, EPA, and TDEC, SWSA SN still has not been officially designated as an Environmental Restoration (ER) concern. Until this is done, no ER expertise can be applied to the problem. Neither a Risk Assessment nor a thorough Remedial Investigation has been or can be performed.

6. Of the three recommendations made in the February 11, 1991, report, the first apparendy has been withdrawn (although no documentation seems to exist); the second has not been implemented; and the third has been implemented.

7. Since the inception of the ASEMP program, the ALs for gross alpha activity, ${ }^{211} \mathrm{Am}$, and ${ }^{2 m} \mathrm{Cm}$ have been exceeded numerous times. The monthly reports do not indicate what actions, if any, were taken as a result of these occurrences. 


\section{$c-12$ \\ ATTACiMINTI 1 \\ DVESTGATION OF TRU WASTE STORAGE AT SWSA SN (continued)}

\section{Footnotes:}

1. These monthly reports did not contain either TRU or gross alpha activity results for SWSA SN.

2. According to Tom Ashwood of ESD, no action level has been established for gross alpha activity in sediments at SWSA SN.

3. Samples tiken from Well 516 on June 26 and August 16, 1990, were acidified prior to filtration. This method was thought to give artificially high gross alpha activity readings, since the scid could have dissolved alpha-emitting constituents in the sediment. The July report further states that "In future sampling, the sequence will be changed to acidification after filuration."

4. This recommendation was not implemented due to the uncertain performance of $\mathrm{MnO}$ fibers. ESD subsequently withdrew the recommendation (per Ashwood, February 10, 1993).

5. Date appens to be in error; should be 11/05/91.

6. The second recommendation called for the resampling of Well 982 and analysis for "TRU elements." The Environmental Surveillance and Protectio'n (ESP) Division at ORNL has been Sampling Wells 982, 983, and 984 annually since August 1990. Although gross alpha activity is an analyte, neither ${ }^{211} \mathrm{Am}$ nor ${ }^{24} \mathrm{Cm}$ are analytes. 
Mr. Larry L. Radcliffe, Director

Waste Management and Technology Development Division

Department of Energy

Oak Ridge Operations Office

Post Office Box 2001

Oak Ridge, Tennessee $37831-8620$

Dear Mr. Radcliffe:

\section{Storage of Transuranic Waste at Solid Waste Storage Area 5 North}

The purpose of this letter is to respond to concerns identified in your letter dated April 9, 1993, on the subject of storage of transuranic (TRU) waste at Solid Waste Storage Area 5 North (SWSA 5N) (Program Manager: L. C. M. Roddye). Subsequent to receipt of your letter, Oak Ridge National Laboratory waste operations staff met with L. C. Roddye of your staff and J. T. Nelson, PAI, on July 22, 1993, to clarify the concerns and define the scope of the response. The following summary presents our responses to the concerns as discussed in that meeting.

1. "Although TRU wastes have been placed in SWSA SN since October 1970, monitoring of groundwater and surface water did not begin until January 1990."

The first requirement to monitor groundwater and surface water at SWSA SN for waste management operations came with the issuance of Deparment of Energy (DOE) Order 5820.2A in September 1988. After review of that order, the Active Sites Environmental Monitoring Program (ASEMP) was developed, and the first samples were collected in November 1989. Since 1986, compliance monitoring at a National Pollutant Discharge Elimination System discharge point on White Oak Creek, which is downstream from SWSA $5 N$, bas been conducted, and no major releases from SWSA $5 N$ or other areas upstream have been detected.

2. "DOE-Waste Management Division is not clear as to the action levels (ALs) prior to the issuance of Revision 1 of the Program Plan (i.e., February 1992). Gross alpha activity in excess of $1 \mathrm{~Bq} / \mathrm{L}$ is the only Al which has come to light so far, and this appears to be undocumented. Likewise. ALs for ${ }^{201} \mathrm{Am}$ and ${ }^{2 m} \mathrm{Cm}$ are said to be any statistically significant detection, but this too appears to be undocumented."

We agree that the use of ALs in the ASEMP is confusing, especially the gross alpha ALs for SWSA 5N. In fact, the levels should be considered as internal reporting levels, rather than ALs because no specific action, other than intermal reporting, is required in the program plan.

In the original ASEMP program plan, an $\mathrm{AL}$ of $1 \mathrm{~Bq} / \mathrm{L}$ was established for all gross alpha measurements. This level was established as part of the tumulus demonstration program and was based on measurements of rain water falling on the empty tumulus pad. After the ASEMP had been active for several months, a new set of ALs was developed based on statistical review of ASEMP data. However, no gross alpha AL was set for SWSA SN because our experience showed that gross alpha measurements were not sufficiently sensitive to detect trace levels of TRU elements. Instead, the measurements of ${ }^{21} \mathrm{Am}$ or ${ }^{24} \mathrm{Cm}$ at concentrations that are statistically greater than the detection limit are considered to have exceeded the AL. This approach was not documented in Revision 1 of the Program Plan. 
3. "The AL for gross alpha activity was exceeded by factors of 170,79, and 9 for samples taken from Well 516 on November 29, 1989, and March 28 and June 26, 1990, respectively. However, these appear to have gone unreported until the October 1990 Occurrence Report."

As discussed under item 2 above, the Als are really internal reporting levels. It was not until October 1990 that sufficient information existed to demonstrate that the elevated gross ALs reflected apparent leakage from the burial trenches and that this leakage represented a reportable occurrence.

4. "No analytical or sample-preparation methods are given in any of the documents reviewed. The concern about when to acidify samples before gross-alpha analyses might not have arisen if standard analytical procedures had been used."

Standard analytical protocols were used by the analytical laboratories involved in the project. Those protocols are documented internally by the laboratories.

The question of when to acidify samples for gross alpha analyses is unrelated to the analytical protocol. The issue is whether one wishes to measure only dissolved contamination (acidify after filtration) or to include contamination that may be adsorbed onto particulate matter (acidify prior to filtration). Because particulate matter in a sample may not represent current conditions within the groundwater aquifer, we have taken the approach that we will only measure dissolved contamination. Hence we have changed our field procedures to include acidification after filtration. This change is documented in the sampling procedure that is part of Revision 1 of the Program Plan.

5. "In spite of numerous attempts by Energy Systems, DOE-Oak Ridge Operations, Environmental Protection Agency, and Tennessee Department of Environment and Conservation, SWSA SN still has not been officially designated as an Environmental Restoration (ER) concern. Until this is done, no ER expertise can be applied to the problem. Neither a risk assessment nor a thorough remedial investigation has been or can be performed."

We agree with the concern, but its resolution is beyond the scope of this response. This issue is being pursued by DOE and Energy Systems with Tennessee Department of Environment and Conservation and Environmental Protection Agency.

6. "Of the three recommendations made in the February 11, 1991, report, the first apparently has been withdrawn (although no documentation seems to exist); the second has not been implemented; and the third has been implemented."

The first recommendation of the February 11, 1991, letter report was to investigate the source of ${ }^{241} \mathrm{Am}$ and ${ }^{24} \mathrm{Cm}$ in White Oak Creek sufficiently to provide stronger evidence that the burial trenches were not the source of those contaminants. This recommendation was not implemented because our data continue to show that ${ }^{24 !} \mathrm{Am}$ and ${ }^{24} \mathrm{Cm}$ from the burial trenches are entering White Oak Creek, though it is important to note that radionuclide release from SWSA SN trenches is not the only or even the largest source. The quantities of ${ }^{241} \mathrm{Am}$ and ${ }^{24} \mathrm{Cm}$ entering White Oak Creek are very small. In addition, it should be noted that the concentrations of radionuclides in water released from the White Oak Creek Dam do not exceed DOE Order 5400.5 release limits.

The second recommendation was inadvertently dropped. Discussions will be held with the Environmental Compliance staff to determine if their wells on the boundary of SWSA SN can be sampled for ${ }^{201} \mathrm{Am}$ and ${ }^{24} \mathrm{Cm}$. 
7. "Since $u=$ inception of the ASEMP program, the ALs for gross alpha activity, ${ }^{241} \mathrm{Am}$, and ${ }^{244} \mathrm{Cm}$ have been exceded numerous times. The monthly reports do not indicate what actions, if any, were taken as a result of these occurrences."

As discussed in the response to item 2 above, the ALs in the ASEMP are really internal reporting levels. The only action required by the program plan is internal reporting. The monthly reports are part of that internal reporting process.

Note: Because the hydrological conditions of the area being monitored are complex and, to a large extent, unknown, the principal function of the monitoring activity is to call attention to atypical conditions for review by qualified staff (as opposed to specifying "automatic" actions to be taken when an "action level" has been exceeded). In this particular case, the recommended action was to continue to monitor the siruation concurrent with performing more investigative studies that would result in a comprehensive understanding of the release of radionuclides to the surface streams from all potential sources in the area. In shor, the results of the investigative studies were to provide the needed basis for determining the correct response action to the monitoring results. After a fairly short effort, it became clear that, from a resources availability standpoint, development of a sufficiently comprehensive understanding of the entire area would have to be carried out under the Environmental Restoration Program as a part of the remediation/closure activities. As noted in item 5 above, the transfer of the facility into the ER Program has not occurred and therefore the information required to provide a basis for a recommended response to the apparent leaching of radionuclides from the SWSA $5 N$ trenches has not yet been developed. However, as stated in item 6 above, the concentrations of radionuclides in water released from the White Oak Creek Dam do not exceed DOE Order 5400.5 release limits.

A summary report of monitoring activities performed at the site will be prepared by March 1994, to serve as a comprehensive record for future reference.

If you have any questions or require additional information, please contact $H$. L. Adair at 574-5901.

Sincerely,

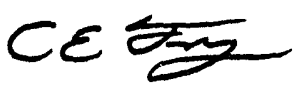

Charles E. Frye, Director

Energy Systems Waste Management

Organization

\section{CEF:HLA:msc}

cc:
H. L. Adair
T. L. Ashwood
G. R. Cunningham
J. T. Nelson, PAI
L. E. McNeese

C. A. Manrod

R. C. Mason

T. F. Scanlan

B. V. Shelton - RC

File - CEF 
February 11, 1991

D. F. Hall

Preliminary Results of Investigation into TRU Contamination in SWSA 5 North Groundwater

We have completed the first phase of the investigation as described in the work plan (Ashwood, T. L., letter to H. L. Adair, 19 October 1990). The purpose of this report is to present the results and our interpretation of those results. We also present recommendations for future activities, but we will not implement those recommendations until we receive your concurrence.

\section{BACKGROUND}

As part of the Active Sites Environmental Monitoring Program (ASEMP), streams and groundwater wells around the TRU waste storage area in Solid Waste Storage Area (SWSA) 5 North are sampled quarterly (Ashwood et al., 1990a). Well 516, immediately down-gradient from a group of TRU waste trenches (Fig. 1), contains gross alpha activity on the order of $150 \mathrm{~Bq} / \mathrm{L}$ (Ashwood et al., 1990b; Wickliff et al., 1991a,b). Curium-244 is the dominant radionuclide, with traces of ${ }^{243} \mathrm{Am}(6 \mathrm{~Bq} / \mathrm{L})$ and ${ }^{241} \mathrm{Am}(0.7 \mathrm{~Bq} / \mathrm{L})$ having been reported from separate samples. The TRU waste trenches also contain some RCRA wastes_primarily lead (Stewart et al., 1989). Samples from well 516 have not contained detectable concentrations of volatile organics. Metal concentrations have been below regulatory concern. The trenches are upgradient from White Oak Creek (WOC) which drains most of ORNL and eventually enters the Clinch River.

Because WOC represents a direct pathway offsite, certain regulatory reporting and corrective action requirements may be invoked if radionuclides or heavy metals have been released to the stream. An action plan for further investigation of the extent of TRU contamination in the groundwater and possibly WOC was developed and implemented.

Phase 1 of the action plan involved review of existing information (primarily the RCRA groundwater wells between SWSA 5 North and WOC), water sampling along the reach of WOC adjacent to SWSA 5 North, sampling of seeps between the TRU burial trenches and WOC. sampling of wells in the vicinity of SWSA 5 North, and installation and analysis of $\mathrm{MnO}_{2}$-coated-fiber samplers at several locations along WOC adjacent to SWSA 5 North. The objective of Phase 1 was to determine whether or not TRU contamination from the TRU burial trenches had reached WOC.

\section{METHODS}

In addition to the routine quarterly samples from wells, seeps, and streams, (Ashwood et al. 1990a, Wickliff et al. 1991a,b), water samples were collected from WOC, wells 708, 715, and 716 , and several seeps not previously sampled (Table 1). Well samples were taken after 1-3 well volumes had been evacuated from the well or the well had been pumped dry and allowed to recover. 
Table 1. Samples collected and analyses performed during Phase 1 investigation in SWSA 5 North

\begin{tabular}{|c|c|c|}
\hline Sample Locationa & Media Sampled & Analyses b \\
\hline 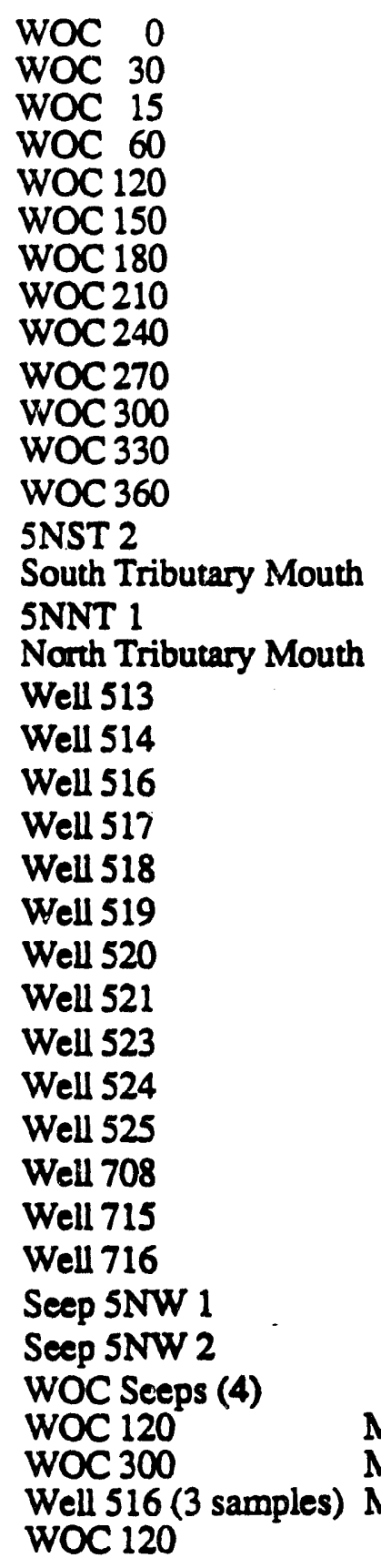 & $\begin{array}{l}\text { Water } \\
\text { Water } \\
\text { Water } \\
\text { Water } \\
\text { Water } \\
\text { Water } \\
\text { Water } \\
\text { Water } \\
\text { Water } \\
\text { Water } \\
\text { Water } \\
\text { Water } \\
\text { Water } \\
\text { Water } \\
\text { Water } \\
\text { Water } \\
\text { Water } \\
\text { Water } \\
\text { Water } \\
\text { Water } \\
\text { Water } \\
\text { Water } \\
\text { Water } \\
\text { Water } \\
\text { Water } \\
\text { Water } \\
\text { Water } \\
\text { Water } \\
\text { Water } \\
\text { Water } \\
\text { Water } \\
\text { Water } \\
\text { Water } \\
\text { Water } \\
\text { MnO2-coated Fiber } \\
\text { MnO2-coated Fiber } \\
\text { Sediment }\end{array}$ & $\begin{array}{l}\text { Gross alpha, gross beta, gamma scan, } 90 \mathrm{Sr} \\
\text { Gross alpha, gross beta, gamma scan } \\
\text { Gross alpha, gross beta, gamma scan } \\
\text { Gross alpha, gross beta, gamma scan } \\
\text { Gross alpha, gross beta, gamma scan } \\
\text { Gross alpha, gross beta, gamma scan } \\
\text { Gross alpha, gross beta, gamma scan } \\
\text { Gross alpha, gross beta, gamma scan } \\
\text { Gross alpha, gross beta, gamma scan } \\
\text { Gross alpha, gross beta, gamma scan, } 90 \mathrm{Sr} \\
\text { Gross alpha, gross beta, gamma scan } \\
\text { Gross alpha, gross beta, gamma scan } \\
\text { Gross alpha, gross beta, gamma scan, } 30 \mathrm{Sr} \\
\text { Gross alpha, gross beta, gamma scan, }{ }^{3} \mathrm{H} \\
\text { Gross alpha, gross beta, gamma scan } \\
\text { Gross alpha, gross beta, gamma scan, } 3 \mathrm{H} \\
\text { Gross alpha, gross beta, gamma scan } \\
\text { Gross alpha, gross beta, gamma scan, } 3 \mathrm{H} \\
\text { Gross alpha, gross beta, gamma scan, } 3 \mathrm{H} \\
\text { Gross alpha, gross beta, gamma scan, } 3 \mathrm{H}, 90 \mathrm{Sr}, \mathrm{ICPC} \\
\text { Gross alpha, gross beta, gamma scan, } 3 \mathrm{H} \\
\text { Gross alpha, gross beta, gamma scan, } 3 \mathrm{H} \\
\text { Gross alpha, gross beta, gamma scan, } 3 \mathrm{H} \\
\text { Gross alpha, gross beta, gamma scan, } 3 \mathrm{H} \\
\text { Gross alpha, gross beta, gamma scan, } 3 \mathrm{H} \\
\text { Gross alpha, gross beta, gamma scan, } 3 \mathrm{H} \\
\text { Gross alpha, gross beta, gamma scan, } 3 \mathrm{H} \\
\text { Gross alpha, gross beta, gamma scan, } 3 \mathrm{H} \\
\text { Gross alpha, gross beta, gamma scan, } 3 \mathrm{H} \\
\text { Gross alpha, gross beta, gamma scan, }{ }^{3} \mathrm{H} \\
\text { Gross alpha, gross beta, gamma scan, } 3 \mathrm{H} \\
\text { Gross alpha, gross beta, gamma scan, }{ }^{3} \mathrm{H} \\
\text { Gross alpha, gross beta, gamma scan, }{ }^{3} \mathrm{H} \\
\text { Gross alpha, gross beta, gamma scan, }{ }^{3} \mathrm{H} \\
\text { Gross alpha, gross beta, gamma scan } \\
\text { Gross alpha, gross beta, gamma scan } \\
\text { Gross alpha, gross beta, gamma scan } \\
\text { Gamma scan }\end{array}$ \\
\hline
\end{tabular}

a See Fig. 1 for locations of samples. WOC $=$ White Oak Creek

b If gross alpha exceeded $1.0 \mathrm{~Bq} / \mathrm{L}$, then transuranic alpha emitters were determined.

c ICP = Inductively Coupled Plasma spectromerry (i.e., metals). 
Gamma counting, gross alpha and gross beta analyses were performed on 1- $L$ samples. Separate $250 \mathrm{~mL}$ samples were collected for ${ }^{3} \mathrm{H}$ and for ${ }^{90} \mathrm{Sr}$ analyses. A $100-\mathrm{mL}$ sample was collected from well 516 for metals analysis. All well samples were filtered through 0.45 micron filters then acidified with $\mathrm{HNO}_{3}$ to $\mathrm{pH}<2$ ( ${ }^{3} \mathrm{H}$ samples were not acidified). WOC and other stream samples were acidified, but not filtered, because we wanted to include the contribution of any activity on suspended particles.

Results from RCRA-compliance wells $982-984$ were obtained from Mark Tardiff (ORNL).

Three 50-g $\mathrm{MnO}_{2}$-coated-fiber samplers were installed in well 516, and ten $100-\mathrm{g}$ $\mathrm{MnO}_{2}$-coated-fiber samplers were installed in WOC and South Tributary (Fig. 1). (Fabrication, installation, and analysis of these samplers are described in the Appendix.) Four days after installation of the samplers in WOC, the area received rainfall equivalent to a 20-year event (D. D. Huff, ORNL, personal communication, 1991), and eight of the samplers were lost in the resultant storm. The two samplers recovered from WOC (Table 1) had collected a large amount of sediment which was washed from the samplers with deionized water.

In order to determine how sediment might affect the results of WOC water and fiber samples, a $125-\mathrm{ml}$ sample of sediment was collected at the WOC 120 site, and a second water sample was taken at WOC 120 and filtered prior to acidification. Both samples were collected on 18 January 1991.

WOC samples (water, sediment, and fiber) and the fiber and water samples from well 516 were gamma-counted in the Environmental Sciences Division (ESD). All other analyses were performed by the Analytical Chemistry Division (ACD). Both ESD and ACD have documented QA/QC programs for the is counting and analytical labs*. Because the samples in this investigation are considered to be screening level, no additional QA/QC actions were taken as part of the investigation.

\section{RESULTS}

Gross alpha activity in water samples was significantly greater than zero (ip < 0.01 ) only in well 516 and the two sample locations on South Tributary (Table 2). This is consistent with all previous ASEMP results (Wickliff et al. 1991a,b). RCRA-compliance well 982 had gross alpha activity of $1 \mathrm{~Bq} / \mathrm{L}$, but no determination was made of the specific isotopes. Only the samples from well 516 contained transuranic elements at activities equivalent to the gross alpha activity (Table 2). Although not specifically determined, the gross alpha activity in South Tributary is most likely caused by $\mathrm{U}$ - or Th-series elements.

After 14 days exposure, each of the $50-\mathrm{g} \mathrm{MnO}_{2}$-coated-fiber samplers in well 516 adsorbed approximately as much gross alpha activity as was found in a 1-L sample from that well (Table 2). Gross alpha activity, including ${ }^{244} \mathrm{Cm}$ and ${ }^{241} \mathrm{Am}$, was found on $\mathrm{MnO}_{2}$-coated-fiber samplers at WOC 120 and 300.

Gross beta activity is significantly greater than zero $(p<0.01)$ throughout WOC and South Tributary, but not in North Tributary. In WOC and at the mouth of South Tributary, gross beta activity approaches the action level of $5.0 \mathrm{~Bq} / \mathrm{L}$. Gross beta activity increases $(\mathrm{p}<0.001)$ between 5NST 2 and the mouth of South Tributary. Although seven wells $(516,517,518,519,524,715$, and 716) have gross beta activity significantly greater than zero $(p<0.01)$, none of the wells approach the action level.

\footnotetext{
"For details on the QAVQC programs, contact I. L. Larsen (ESD) and J. R. Stokely, Jr. (ACD).
} 
Table 2. Gross alpha, gross beta, ${ }^{3} \mathrm{H},{ }^{90} \mathrm{Sr}$, and transuranic activitiesa in SWSA 5 North and White Oak Creek samples

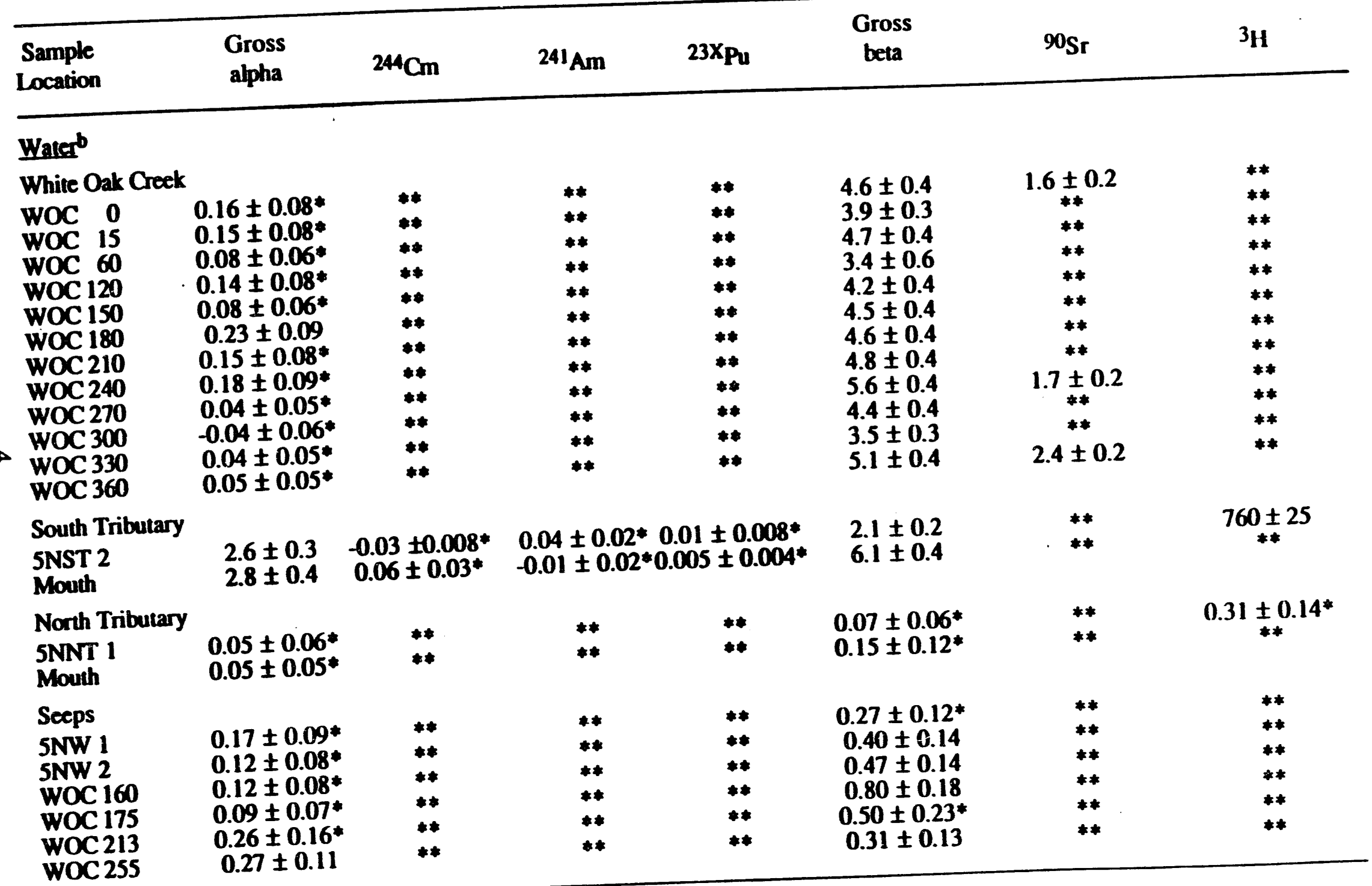


Table 2 (continued). Gross alpha, gross beta, ${ }^{3} \mathrm{H},{ }^{90} \mathrm{Sr}$, and transuranic activitiesa in SWSA 5 North and White Oak Creek samples

\begin{tabular}{|c|c|c|c|c|c|c|c|}
\hline $\begin{array}{l}\text { Sample } \\
\text { Location }\end{array}$ & $\begin{array}{c}\text { Gross } \\
\text { alpha }\end{array}$ & ${ }^{244} \mathrm{Cm}$ & $241 \mathrm{Am}$ & ${ }^{23 X} \mathrm{Pu}$ & $\begin{array}{c}\text { Gross } \\
\text { beta }\end{array}$ & ${ }^{90} \mathrm{Sr}$ & 311 \\
\hline $\begin{array}{l}\text { Wells } \\
513 \\
514 \\
516 \\
517 \\
518 \\
519 \\
520 \\
521 \\
523 \\
524 \\
525 \\
708 \\
715 \\
716 \\
982 \\
983 \\
984\end{array}$ & $\begin{array}{c}0.02 \pm 0.02^{*} \\
0.035 \pm 0.036^{*} \\
38 \pm 1 \\
0.023 \pm 0.041^{*} \\
0.14 \pm 0.05 \\
0.01 \pm 0.04^{*} \\
0.05 \pm 0.06^{*} \\
0.0 \pm 0.05^{*} \\
0.15 \pm 0.08^{*} \\
-0.01 \pm 0.03^{*} \\
0.05 \pm 0.06^{*} \\
0.01 \pm 0.02^{*} \\
0.03 \pm 0.03^{*} \\
0.04 \pm 0.03^{*} \\
1.1 \\
* * * \\
* * *\end{array}$ & $\begin{array}{c}* * \\
* * \\
41 \pm 2 \\
* * \\
* * \\
* * \\
* * \\
* * \\
* * \\
* * \\
* * \\
* * \\
* * \\
* * \\
* * \\
* * \\
* *\end{array}$ & $\begin{array}{c}* * \\
* * \\
1.7 \pm 0.5 \\
* * \\
* * \\
* * \\
* * \\
* * \\
* * \\
* * \\
* * \\
* * \\
* * \\
* * \\
* * \\
* * \\
* *\end{array}$ & $\begin{array}{c}* * \\
* * \\
0.11 \pm 0.04 \\
* * \\
* * \\
* * \\
* * \\
* * \\
* * \\
* * \\
* * \\
* * \\
* * \\
* * \\
* * \\
* *\end{array}$ & $\begin{array}{c}0.01 \pm 0.06^{*} \\
0.04 \pm 0.1^{*} \\
2.2 \pm 0.2 \\
0.28 \pm 0.11 \\
0.32 \pm 0.08 \\
0.3 \pm 0.11 \\
0.08 \pm 0.1 * \\
0.12 \pm 0.08^{*} \\
0.22 \pm 0.18^{*} \\
0.35 \pm 0.12 \\
0.17 \pm 0.13^{*} \\
0.10 \pm 0.06^{*} \\
0.6 \pm 0.12 \\
0.27 \pm 0.08 \\
* * * \\
* * * \\
* * *\end{array}$ & $\begin{aligned} * * \\
* * \\
0.054 \pm 0.034 * \\
* * \\
* * \\
* * \\
* * \\
* * \\
* * \\
* * \\
* * \\
* * \\
* * \\
* * \\
* * * \\
* * * \\
* * *\end{aligned}$ & $\begin{array}{c}25 \pm 18^{*} \\
33 \pm 17^{*} \\
51 \pm 12 \\
37 \pm 17^{*} \\
22 \pm 18^{*} \\
27 \pm 17^{*} \\
36 \pm 12 \\
-45 \pm 16^{*} \\
55 \pm 18 \\
370 \pm 22 \\
2 \pm 10^{*} \\
220 \pm 20 \\
10 \pm 16^{*} \\
-30 \pm 17^{*} \\
* * * \\
* * * \\
86\end{array}$ \\
\hline $\begin{array}{l}\text { Fiber } \\
\text { WOC } 120 \\
\text { WOC } 300 \\
\text { Well } 516 \\
\text { Blanke }\end{array}$ & $\begin{array}{l}* * \\
* * \\
* * \\
* *\end{array}$ & $\begin{array}{c}0.044 \pm 0.006 \\
0.052 \pm 0.007 \\
32.8 \pm 2.02^{d} \\
<0.002\end{array}$ & $\begin{array}{c}0.053 \pm 0.007 \\
0.080 \pm 0.009 \\
* * \\
<0.002\end{array}$ & $\begin{array}{l}* * \\
* * \\
* * \\
* *\end{array}$ & $\begin{array}{l}* * \\
* * \\
* * \\
* *\end{array}$ & $\begin{array}{l}* * \\
* * \\
* * \\
* *\end{array}$ & $\begin{array}{l}* * \\
* * \\
* * \\
* *\end{array}$ \\
\hline
\end{tabular}

Results are presented as mean plus 1 standard error (counting error only).

b Results are in $\mathrm{Bc} / \mathrm{L}$.

c Results are in Bq/sample

d Results are mean of three replicate samples \pm standard error of the mean.

e A $50-\mathrm{g}$ sample of fiber was soaked for $-16 \mathrm{~h}$ in deionized water and submitted to ACD as a blind blank.

* Value is not significantly greater than $0.00(p<0.01)$

** Analysis not performed

*** Value not reponted 
One cause of the gross beta activity in WOC is ${ }^{90} \mathrm{Sr}$ (Table 2). A tributary draining SWSA 4 enters WOC just below WOC 300 (Fig. 1), and SWSA 4 is suspected to be a major source of ${ }^{90} \mathrm{Sr}$ (ESD 1990). Nevertheless, the difference in $90 \mathrm{Sr}$ activity between WOC 270 and WOC 360 is only marginally greater than might be expected from random counting error $(0.05>p>0.01)$.

Tritium activity is significantly greater than zero $(\mathrm{p}<0.01)$ in wells $516,520,523,524,708$, and 984 , and in South Tributary (Table 2). Tritium is not a contributor to gross beta activity because concentration procedures in the gross beta analysis cause the tritium to evaporate.

None of the wells had ${ }^{60} \mathrm{Co}$ or ${ }^{137} \mathrm{Cs}$ activities that were significantly greater than zero $(\mathrm{p}<0.01)$ (Table 3). WOC samples contained ${ }^{137} \mathrm{Cs}$ but no ${ }^{60} \mathrm{Co}$. Neither tributary contained ${ }^{60} \mathrm{Co}$ or ${ }^{137}$ Cs.

Table 3. Gamma-scan results ${ }^{a}$ from SWSA 5 North water, fiber, and sediment samples

\begin{tabular}{|c|c|c|c|c|c|}
\hline $\begin{array}{l}\text { Sample } \\
\text { Location }\end{array}$ & ${ }^{40} \mathrm{~K}$ & ${ }^{60} \mathrm{Co}$ & ${ }^{137} \mathrm{Cs}$ & ${ }^{152} \mathrm{Eu}$ & ${ }^{154} \mathrm{Eu}$ \\
\hline \multicolumn{6}{|l|}{ Water $^{b}$} \\
\hline \multicolumn{6}{|c|}{ White Oak Creek } \\
\hline $\begin{array}{lr}\text { WOC } & 0 \\
\text { WOC } & 15 \\
\text { WOC } 60 \\
\text { WOC } 120 \\
\text { WOC } 120-2 c \\
\text { WOC } 150 \\
\text { WOC } 180 \\
\text { WOC } 210 \\
\text { WOC } 240 \\
\text { WOC } 270 \\
\text { WOC } 300 \\
\text { WOC } 330 \\
\text { WOC } 360\end{array}$ & $\begin{aligned} 1.94 & \pm 0.4 \\
& <4 \\
& <4 \\
& <1 \\
& <1 \\
& <2 \\
& <4 \\
& <4 \\
& <4 \\
& <2 \\
& <1 \\
0.04 & \pm 0.96 * \\
& <2\end{aligned}$ & $\begin{array}{c}<0.1 \\
<0.2 \\
<0.2 \\
0.06 \pm 0.07^{*} \\
0.03 \pm 0.02^{*} \\
<0.1 \\
<0.2 \\
<0.2 \\
0.1 \pm 0.06^{*} \\
<0.1 \\
<0.1 \\
<0.2 \\
<0.1\end{array}$ & $\begin{array}{c}1.73 \pm 0.07 \\
1.67 \pm 0.11 \\
1.67 \pm 0.11 \\
15.37 \pm 0.12 \\
0.78 \pm 0.04 \\
1.86 \pm 0.07 \\
1.59 \pm 0.10 \\
1.70 \pm 0.10 \\
1.53 \pm 0.07 \\
2.02 \pm 0.06 \\
1.47 \pm 0.04 \\
1.58 \pm 0.13 \\
1.48 \pm 0.06\end{array}$ & $\begin{array}{l}<0.5 \\
<0.5 \\
<0.5 \\
<0.5 \\
<0.5 \\
<0.5 \\
<0.5 \\
<0.5 \\
<0.5 \\
<0.5 \\
<0.5 \\
<0.5 \\
<0.5\end{array}$ & $\begin{array}{l}<0.5 \\
<0.5 \\
<0.5 \\
<0.5 \\
<0.5 \\
<0.5 \\
<0.5 \\
<0.5 \\
<0.5 \\
<0.5 \\
<0.5 \\
<0.5 \\
<0.5\end{array}$ \\
\hline $\begin{array}{l}\text { North Tributar } \\
\text { 5NNT } 1 \\
\text { Mouth }\end{array}$ & $\begin{array}{c}7.4 \pm 1.0 \\
1.54 \pm 0.71 *\end{array}$ & $\begin{array}{c}-0.01 \pm 0.21 * \\
<0.1\end{array}$ & $\begin{array}{c}0.01 \pm 0.21 * \\
<0.1\end{array}$ & $\begin{array}{l}* * * * \\
<0.5\end{array}$ & $\begin{array}{l}* * * * \\
<0.5\end{array}$ \\
\hline $\begin{array}{l}\text { South Tributar } \\
\text { 5NST } 2 \\
\text { Mouth }\end{array}$ & $\underset{<1}{4.5 \pm 1.6}$ & $\begin{array}{c}-0.02 \pm 0.27 * \\
<0.1\end{array}$ & $\begin{array}{c}0.08 \pm 0.22 \\
<0.1\end{array}$ & $\begin{array}{l}* * * \\
<0.5\end{array}$ & $<0.5$ \\
\hline $\begin{array}{l}\text { Seeps } \\
\text { 5NW } 1 \\
\text { 5NW } 2 \\
\text { WOC } 160 \\
\text { WOC } 175 \\
\text { WOC } 213 \\
\text { WOC } 255\end{array}$ & $\begin{aligned} 11.3 & \pm 1.78 \\
& <1 \\
& <1 \\
& <1 \\
0.94 & \pm 1 \\
& \pm 1.13^{*}\end{aligned}$ & $\begin{array}{l}<0.5 \\
<0.5 \\
<0.5 \\
<0.5 \\
<0.5 \\
<0.5\end{array}$ & $\begin{aligned} & <0.5 \\
0.13 & \pm 0.12 * \\
& <0.5 \\
& <0.5 \\
& <0.5 \\
& <0.5\end{aligned}$ & $\begin{array}{l}<0.5 \\
<0.5 \\
<0.5 \\
<0.5 \\
<0.5 \\
<0.5\end{array}$ & $\begin{array}{l}<0.5 \\
<0.5 \\
<0.5 \\
<0.5 \\
<0.5 \\
<0.5\end{array}$ \\
\hline
\end{tabular}


Table 3 (continued). Gamma-scan results from SWSA 5 North water, fiber, and sediment samples

\begin{tabular}{|c|c|c|c|c|c|}
\hline $\begin{array}{l}\text { Sample } \\
\text { Location }\end{array}$ & ${ }^{40} \mathrm{~K}$ & ${ }^{60} \mathrm{Co}$ & ${ }^{137} \mathrm{Cs}$ & $152 \mathrm{Eu}$ & ${ }^{154} \mathrm{E}_{1}$ \\
\hline \multicolumn{6}{|l|}{ Wells } \\
\hline $\begin{array}{l}513 \\
514 \\
516 \\
517 \\
518 \\
519 \\
520 \\
521 \\
523 \\
524 \\
525\end{array}$ & $\begin{array}{c}<1 \\
* * * \\
1.30 \pm 0.55 \\
* * * \\
1.1 \pm 1.4^{*} \\
* * * \\
7.2 \pm 1.0 \\
1.0 \pm 1.2^{*} \\
* * * \\
* * * \\
12 \pm 1\end{array}$ & $\begin{array}{c}-0.08 \pm 0.23^{*} \\
0.06 \pm 0.31^{*} \\
0.32 \pm 0.91^{*} \\
-0.11 \pm 0.33^{*} \\
0.08 \pm 0.28^{*} \\
0.05 \pm 0.28^{*} \\
0.12 \pm 0.18^{*} \\
0.04 \pm 0.24^{*} \\
0.10 \pm 0.19^{*} \\
-0.02 \pm 0.24^{*} \\
-0.06 \pm 0.24^{*}\end{array}$ & $\begin{array}{c}-0.08 \pm 0.19^{*} \\
0.12 \pm 0.25^{*} \\
<0.1 \\
0.05 \pm 0.30 \\
0.05 \pm 0.31 \\
0.18 \pm 0.27^{*} \\
0.02 \pm 0.18 \\
-0.04 \pm 0.24^{*} \\
-0.04 \pm 0.18^{*} \\
0.04 \pm 0.24^{*} \\
0.11 \pm 0.21^{*}\end{array}$ & $\begin{array}{l}* * * \\
* * * \\
<0.2 \\
* * * \\
* * * \\
* * * \\
* * * \\
* * * \\
* * * \\
* * * \\
* * *\end{array}$ & $\begin{array}{l}* * * \\
* * * \\
<0.2 \\
* * * \\
* * * \\
* * * \\
* * * \\
* * * \\
* * * \\
* * * \\
* * *\end{array}$ \\
\hline \multicolumn{6}{|l|}{ Wells } \\
\hline $\begin{array}{l}708 \\
715 \\
716 \\
982 \\
983 \\
984\end{array}$ & 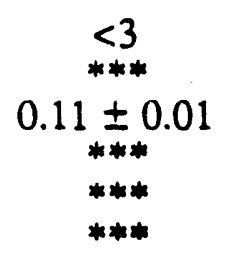 & $\begin{array}{c}0.07 \pm 0.21^{*} \\
-0.09 \pm 0.34^{*} \\
0.11 \pm 0.19^{*} \\
* * * \\
* * * \\
* * *\end{array}$ & $\begin{array}{c}0.15 \pm 0.12^{*} \\
-0.03 \pm 0.28^{*} \\
0.09 \pm 0.20^{*} \\
* * * \\
0.31 \\
* * *\end{array}$ & $\begin{array}{l}* * * \\
* * * \\
* * * \\
* * * \\
* * * \\
* * *\end{array}$ & $\begin{array}{l}* * * \\
* * * \\
* * * \\
* * * \\
* * * \\
* * *\end{array}$ \\
\hline \multicolumn{6}{|l|}{ Eiberd } \\
\hline $\begin{array}{l}\text { WOC } 120 \\
\text { WOC } 300 \\
\text { Well 516e }\end{array}$ & $\begin{array}{c}12.2 \pm 0.44 \\
11.3 \pm 0.56 \\
5.4 \pm 0.90\end{array}$ & $\begin{array}{c}1.15 \pm 0.04 \\
2.44 \pm 0.07 \\
<0.01\end{array}$ & $\begin{array}{c}106 \pm 0.22 \\
333 \pm 0.37 \\
0.037 \pm 0.01\end{array}$ & $\begin{array}{c}0.63 \pm 0.07 \\
12.2 \pm 0.37 \\
<0.2\end{array}$ & $\begin{array}{c}1.48 \pm 0.37 \\
4.82 \pm 0.74 \\
<0.2\end{array}$ \\
\hline \multicolumn{6}{|l|}{ Sediment } \\
\hline
\end{tabular}

a Results are presented as mean plus 1 standard error (counting error only).

$b$ Results are in Bq/L.

c A second sample was collected from WOC 120 and filtered prior to acidification.

d Results are in Bq/sample

e Results are mean of three replicate samples \pm standard error of mean.

f Results are in Bq/g dry weight.

* Value is not significantly greater than $0.00(p<0.01)$

** Analysis not performed

*** Value not reported

Barium and chromium were the only RCRA-regulated metals detected in well 516 (Table 4). The levels of these metals is below regulatory concern ( $1 \mathrm{mg} / \mathrm{L}$ for $\mathrm{Ba}$ and $5 \mathrm{mg} / \mathrm{for} \mathrm{Cr}$ ). 
Table 4. Metal concentrations in well 516.

\begin{tabular}{lc}
\hline Metal & Concentration (mg/L) \\
\hline $\mathrm{Ag}$ & $<0.005$ \\
$\mathrm{As}$ & $<0.05$ \\
$\mathrm{Ba}$ & 0.40 \\
$\mathrm{Ca}$ & 130 \\
$\mathrm{Cd}$ & $<0.005$ \\
$\mathrm{Co}$ & $<0.004$ \\
$\mathrm{Cr}$ & 0.008 \\
$\mathrm{Cu}$ & $<0.007$ \\
$\mathrm{Fe}$ & $<0.05$ \\
$\mathrm{Mg}$ & 16 \\
$\mathrm{Mn}$ & $<0.001$ \\
$\mathrm{Mo}$ & $<0.04$ \\
$\mathrm{Na}$ & 12 \\
$\mathrm{Ni}$ & 0.004 \\
$\mathrm{~Pb}$ & $<0.05$ \\
$\mathrm{Se}$ & $<0.05$ \\
$\mathrm{Si}$ & 8.6 \\
$\mathrm{Sn}$ & $<0.05$ \\
$\mathrm{Sr}$ & 0.20 \\
$\mathrm{Zn}$ & $<0.005$ \\
\hline
\end{tabular}

\section{DISCUSSION}

The most important result of this investigation is that there is both ${ }^{244} \mathrm{Cm}$ and ${ }^{241} \mathrm{Am}$ activity in the reach of WOC adjicent to SWSA 5 North. The source of this transuranic activity is unclear at this stage of the investigation. The activity was only detected using alpha spectrometry on the fiber samples (Appendix). Whereas the ${ }^{244} \mathrm{Cm}$ activity in well 516 is -25 times greater than the ${ }^{241} \mathrm{Am}$ activity, the ${ }^{244} \mathrm{Cm}$ and ${ }^{241} \mathrm{Am}$ activities on the fiber samples are roughly equivalent. Unless there is a preferential adsorption of ${ }^{241} \mathrm{Am}$ on the fibers, this difference in activity ratios would suggest that the WOC transuranics have another source besides the burial trenches in SWSA 5 North.

Comparing the results from WOC 120 and WOC 120-2 water samples (Table 3) suggests that suspended sediment contributed $-95 \%$ of the ${ }^{137} \mathrm{Cs}$ activity in the first sample. The importance of suspended sediment is further illustrated by the results for the sediment sample from WOC 120. Entrainment of $6.6 \mathrm{~g}$ of sediment in the fiber sampler at WOC 120 would account for the activity of $60 \mathrm{Co},{ }^{137} \mathrm{Cs}$, an 152 Eu measured in that sample. Although attempts were made to remove sediment from fiber samples, it is not inconceivable that a small amount remained. High ${ }^{40} \mathrm{~K}$ activities in fiber sampies should be expected because the fibers are prepared in a $\mathrm{KMnO}_{4}$ solution.

Because the gamma activity in the fiber samples is probably caused by entrained sediment, it is possible that sediment is also the source of the transuranic activity. Given the largely unknown sediment dynamics in WOC, especially during the 20-year storm that occurred during the sampling period, it is impossible to conclusively identify the source of sediment contamination. 
South Tributary is clearly receiving some input of both alpha- and beta-emitting isotopes between 5NST 2 and the mouth. The cause of the alpha contamination is not transuranic elements, which suggests that the source is not in SWSA 5 North.

\section{RECOMMENDATIONS}

Three actions appear appropriate at this point in the investigation. First, the source of ${ }^{244} \mathrm{Cm}$ and $241 \mathrm{Am}$ in WOC should be investigated enough to provide stronger evidence that these contaminants are not coming from the burial trenches. Specifically, large-volume (e.g., 100-L) water samples should be collected at four or five locations along WOC, filtered, concentrated (possibly using MnO-coated fibers in a column), and analyzed for transuranic elements. The filtered sediment at each location would also be analyzed for transuranic elements. This step would accomplish two objectives: (1) quantify the effect of suspended sediment on the concentration of transuranic elements in WOC, and (2) determine whether TRU contaminants enter WOC in the reach adjacent to SWSA 5 North.

The second step is to request that well 982 be resampled and analyzed for transuranic elements. Such a request should be made to John Murphy soon, because their next scheduled round of sampling for those wells is approaching.

Our third recommendation is to add the four new seep sites and wells 708 and 715 to the regular ASEMP quarerly sampling protocol.

The action plan contained an outline of possible phase 2 investigations (e.g., tracer tests, additional wells, and soil sampling). It may be appropriate to consider some of those actions at this time, although we recommend that the above three steps be implemented first. It may be more cost effective to conduct further investigations as part of the RI/FS for WAG 5. In any case, our data should be shared with the RI/FS team so that they can incorporate these results into their planning.

We would appreciate an early opportunity to discuss the results and recommendations in this report with you and anyone else you suggest.

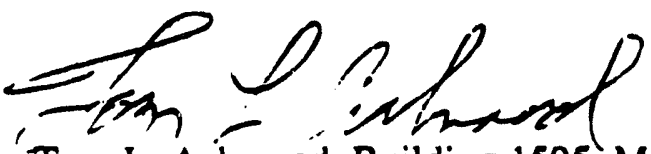

'Tom L. Ashwood, Building 1505, MS 6036, ORNL (4-7542)

Ciume S Wicklify
Diana S. Wickliff, Building 1505, MS 6036, ORNL (4-7380)

TLA:DSW:ta

cc: H. L. Adair

H. L. Boston

R. B. Clapp

S. B. Garland

D. D. Huff

C. P. Manrod

C. M. Morrissey
T. F. Scanlan

R. L. Siegrist

S. H. Stow

J. E. Van Cleve

C. Whitmire

L. C. Williams 


\section{REFERENCES}

Ashwood, T. L., D. S. Wickliff, and C. M. Morrissey. 1990a. Active sites environmental monitoring program: program plan. ORNL/M-1197. $28 \mathrm{p}$.

Ashwood, T. L., D. S. Wickliff, C. M. Morrissey, and H. L. Adair. 1990b. Active sites monitoring at Oak Ridge National Laboratory. pp 397-399 IN Proceedings of SPECTRUM 90 Nuclear and Hazardous Waste Management International Topical Meeting, September 30. October 4, 1990, Knoxville, Tennessee. American Nuclear Society, La Grange Park, Illinois.

Environmental Sciences Division (ESD). 1990. Remedial action plan for waste area grouping 2 at Oak Ridge National Laboratory, Oak Ridge, Tennessee. ES/ER-14\&D1.

Stewart, R. C., I. S. Dickerson, S. F. Joost, and D. C. Osucha. 1989. Remote-handled transuranic solid waste characterization study: Oak Ridge National Laboratory. ORNL/TM11050. $94 \mathrm{p}$.

Wickliff, D. S., C. M. Morrissey, and T. L. Ashwood. 1991a. Active sites environmental monitoring program: Mid-FY 1990 summary report. ORNLM-1179. IN PRESS

Wickliff, D. S., C. M. Morrissey, and T. L. Ashwood. 1991b. Active sites environmental monitoring program: FY 1990 annual report. IN PRESS 


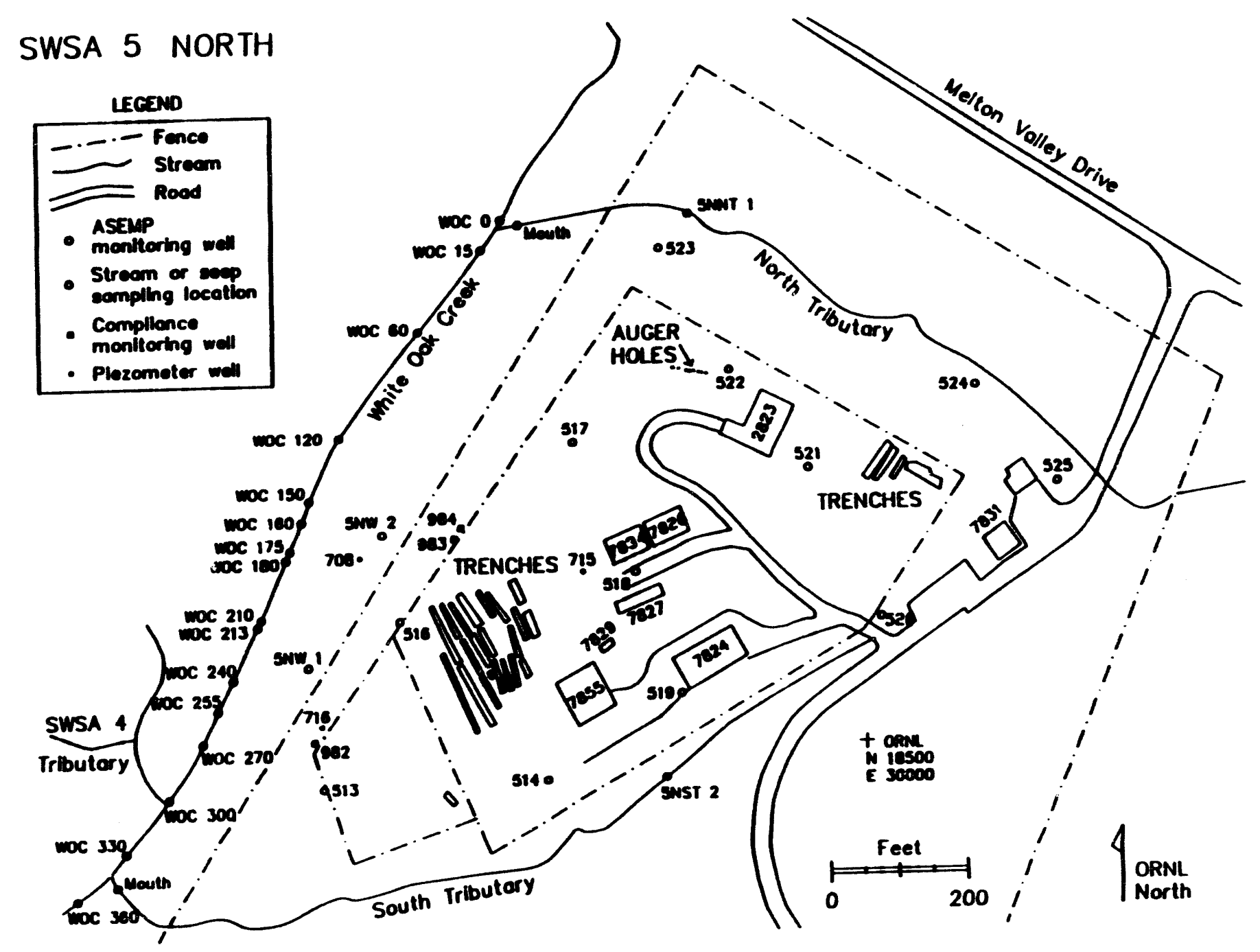

I.ig. 1. TRU waste storage areas and sampling locations in and around SWSA 5 North. 


$$
\text { c-27 }
$$

\section{APPENDIX}

FABRICATION, INSTALLATION, AND ANALYSIS OF $\mathrm{MnO}_{2}$-COATED-FIBER SAMPLERS 


\section{INTRODUCTION}

The use of $\mathrm{MnO}_{2}$-coated fibers to remove $\mathrm{Ra}$ from sea and fresh water has been repeatedly demonstrated (Moore and Reid 1973, Moore and Cook 1975, Moore et al. 1985). The potential for removal of other radionuclides on these fibers is high (W. S. Moore, Univ. South Carolina, personal communication, 1990). Cerling and Spalding (1982) demonstrated that ${ }^{60}$ Co was bound up

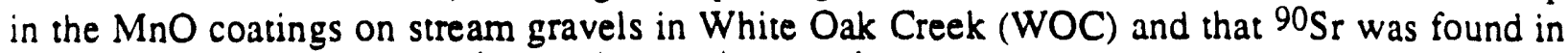
these same coatings or on exchange sites on the gravels.

\section{FABRICATION AND INSTALLATION}

Acrylic fibers were prepared in a potassium-permanganate solution as described by Moore et al. (1985). For this study, $\mathrm{MnO}_{2}$-coated fibers were supplied by W. S. Moore (Univ. South Carolina).

Fibers were encased in a nylon mesh sheath. Two different sizes were used: $-50 \mathrm{~g}$ of fiber were used for each sample in well 516 and for a blank sample, and $-100 \mathrm{~g}$ of fiber were used in the WOC samplers. The sheathed fibers were placed in plastic bottles which had been drilled with several $0.5-\mathrm{cm}$ holes.

Three 50-g sheaths were placed in $250-\mathrm{ml}$ bottles. The bottles were suspended on a nylon cord at approximately $0.3-\mathrm{m}$ intervals and lowered into well 516 so that the uppermost bottle was below the water surface in the well. The bottles were installed in well 516 on 14 Dec 1990 and were removed three weeks later on 4 Jan 1991.

Ten $100-g$ sheaths were placed in $1-\mathrm{L}$ bottles and installed at locations along WOC (Table 1$)$. Nine of the bottles were anchored by nylon cord between two bricks on the bottom of the creek. At WOC 300 the water was too deep for this approach, so the bottle was suspended on nylon cord from a tree limb and weighted with a single brick. At each WOC location, water parameters were measured just after installation of the bottles (Table 1). Samplers were installed on 19 Dec 1990. On $8 \mathrm{Jan}$ 1991, an attempt was made to recover the samplers. However, the 20-year storm that occurred on 23 Dec 1990 apparently washed all but two of the samplers (WOC 120 and WOC 300) away.

Table 1. Sample locations and water parameters for installation of $\mathrm{MnO}_{2}$-coated-fiber samplers

\begin{tabular}{lrcll}
\hline Location & Sampler no. & Temperature $\left({ }^{\circ} \mathrm{C}\right)$ & $\mathrm{pH}$ & Specific conductance (mmhos) \\
\hline WOC 0 & 9 & 14.44 & 7.22 & 0.372 \\
WOC 120 & 10 & 14.50 & 7.15 & 0.365 \\
WOC 180 & 7 & 14.33 & 7.25 & 0.370 \\
WOC 195 & 11 & 14.30 & 7.24 & 0.370 \\
WOC 210 & 13 & 14.28 & 7.26 & 0.374 \\
WOC 225 & 4 & 14.26 & 7.26 & 0.374 \\
WOC 240 & 6 & 14.25 & 7.26 & 0.375 \\
WOC 270 & 12 & 14.22 & 7.26 & 0.374 \\
WOC 300 & 8 & 14.20 & 7.26 & 0.375 \\
South Tributary & 5 & 10.72 & 6.92 & 0.367 \\
\hline
\end{tabular}




\section{ANALYSIS}

After collection, all samples were washed twice with distilled water to remove as much suspended sediment as possible. Nevertheless, is is possible that a small amount of sediment remained embedded in the fibers.

Fibers were subjected to two analyses: gamma counting and alpha spectrometry. Gamma counting was accomplished in the Environmental Sciences Division counting rooms. Samples were squeezed to remove excess moisture prior to counting. The 50-g samples were placed in 125-cc plastic jars for counting. The 100-g samples were placed inio 500-ml Maranelli beakers for counting.

After gamma-counting, samples were transferred to the Analytical Chemistry Division (ACD) for alpha analysis (see attached letter from J. Wade). A 50-g blank sample of fiber was prepared by allowing the fiber to stand in deionized water overnight. This sample was placed in a 125-cc plastic jar and labelled 516-4 so that it would serve as a blind blank to ACD.

\section{REFERENCES}

Cerling, T. E., and B. P. Spalding. 1982. Distribution and relationship of radionuci.des to streambed gravels in a small watershed. Environ. Geol 4:99-116.

Moore, W. S., and D. F. Reid. 1973. Extraction of radium from natural waters using manganese-impregnated acrylic fibers. LGeophys. Res. 78(36):8880-8886.

Moore, W. S., and L. M. Cook. 1975. Radium removal from drinking water. Nature 253:262263.

Moore, W. S., R. M. Key, and J. L. Sarmiento. 1985. Techniques for precise mapping of $226 \mathrm{Ra}$ and ${ }^{228} \mathrm{Ra}$ in the ocean. L. Geophys. Res, 90(C4):6983-6994. 
February 6, 1991

Tom Ashwood

Description of Analytical Methodolory for Analysis of Acrulic Fibers

A "blank" sample of tiber weighing $45.2 \mathrm{~g}$ was placed in a beaker and covered with water. Aliquots of Am-243 and Cm-244 were spiked into the beaker and mired with the sample and water. A pH probe was inserted into the beaker to assure that a pH of at least 7 was obtained. The pH was 8.8. The sample was agitated several times during the day to ensure good contact between the radiotracers and the fiber. The next morning the fiber was removed from the beaker and all excess water was squeezed from it. The fiber was placed in a clean beaker and covered with $2 \mathrm{M}$ nitric acid. The fiber was agitated several times during the leaching time of $2 \mathrm{hrs}$. The fiber was removed and all acid squeezed from it and the leach was repeated. The leaches were combined for a total volume of 300 $\mathrm{ml}$. The sample was placed on a hot plate and taken to dryness two times with concentrated nitric acid. The whitish residue was taken up in a solution of nitric acid and aluminum nitrate and put through an ion-exchange column (TRU-Spec from EIChrom Industries). The americium and curium was eluted from the column with weak nitric acid, and the eluate was transferred to a stainless steel disc. The disc was counted to ascertain the spike recoveries, which were found to be $>90 \%$.

The three samples from the contaminated well were leached with nitric acid as above and a spike of Am-243 was added to monitor chemical recovery. The radionuclides were separated and determined as above and found to be:

\section{$\mathrm{Cm}-244$}

$$
\begin{array}{ll}
\text { Well 516-1 } & 31 \pm 1 \text { Bq/TOT } \\
\text { Well 516-2 } & 37 \pm 2 \text { Bq/TOT } \\
\text { Well 516-3 } & 25 \pm 1 \text { Bq/TOT }
\end{array}
$$

After these three were leached, the fiber was ashed and diseolved in hydroxylamine hydrochloride. A spike of Am-243 was added and the radionuclides were separated and determined as above. The Cm-244 was found to be:

$$
\begin{array}{ll}
\text { Well 516-1 } & 1.6 \mathrm{~Bq} / \mathrm{TOT} \\
\text { Well 516-2 } & 1.9 \mathrm{~Bq} / \mathrm{TOT} \\
\text { Well 516-3 } & 1.8 \mathrm{~Bq} / \mathrm{TOT}
\end{array}
$$

Therefore, over $95 \%$ of the $\mathrm{Cm}-244$ was extracted from the fibers with the nitric acid leach. 
C-31

Tom Astrwood

$-2-$

February 6, 1991

Three more samples were leached with nitric acid and processed as above and found to have the following radionuclide concentrations:

Am-241

WOC Site 8

WOW Site 10

Well $516-4$
$.080 \pm .009$

$.053 \pm .007$

$\leq .002$
Cm-244

$.052 \pm .007 \quad \mathrm{~Bq} / \mathrm{TOT}$

$.044 \pm .006$

Bq/TOT

$\leq .002$

Bq/TOT

afóxic

J. W. Wade

JWWadc 


\section{INTERNAL DISTRIBUTION}

1-5. T. L. Ashwood

6. L. D. Bates

7. H. L. Boston

8-13. G. R. Cunningham

14. J. H. Cushman

15. C. W. Gehrs

16. D. F. Hall

17. S. E. Herbes

18. D. S. Hicks

19. S. G. Hildebrand

20. P. Kanciruk

21. R. H. Ketelle

22. C. A. Manrod

23-28. J. D. Marsh, Jr.

29. J. F. McCarthy
30. C. M. Morrissey

31. J. B. Murphy

32. C. E. Nix

33. D. E. Reichle

34. T. F. Scanlan

35. F. E. Sharples

36. D. S. Shriner

37. S. H. Stow

38. Central Research Library

39-48. ESD Library

49-50. Laboratory Records Dept.

51. Laboratory Records, ORNL-RC

52. ORNL Patent Section

53. ORNL Y-12 Technical Library

\section{EXTERNAL DISTRIBUTION}

54. R. N. Farvolden, Professor, Department of Earth Sciences, University of Waterloo, Waterloo, Ontario N2L 3G1 Canada 55. D. W. Freckman, Director, College of Natural Resources, 101 Natural Resources
Building, Colorado State University, Fort Collins, CO 80523

56. R. C. Harriss, Institute for the Study of Earth, Oceans, and Space, Science and Engineering Research Building, University of New Hampshire, Durham, NH 03824

57. G. Y. Jordy, Director, Office of Program Analysis, Office of Energy Research ER-30, G-226, U.S. Department of Energy, Washington, DC 20545

58. A. Patrinos, Director, Environmental Sciences Division, Office of Health and Environmental Research, ER-74, U.S. Department of Energy, Washington, DC 20585

59. L. L. Radcliffe, Director, Waste Management and Technology Development Division, U.S. Department of Energy, Oak Ridge Operations Office, P.O. Box 2001, Oak Ridge, TN 37831-8620

60. L. C. M. Roddye, Program Manager, Waste Management and Technology Development Division, U.S. Department of Energy, Oak Ridge Operations Office, P.O. Box 2001, Oak Ridge, TN 37831-8620

61. F. J. Wobber, Environmental Sciences Division, Office of Health and Environmental Research, ER-74, U.S. Department of Energy, Washington, DC 20585

62. Office of Assistant Manager for Energy Research and Development, U.S. Department of Energy Oak Ridge Operations, P.O. Box 2001, Oak Ridge, TN 37831-8600.

63-64. Office of Scientific and Technical Information, P.O. Box 62, Oak Ridge, TN 37831 

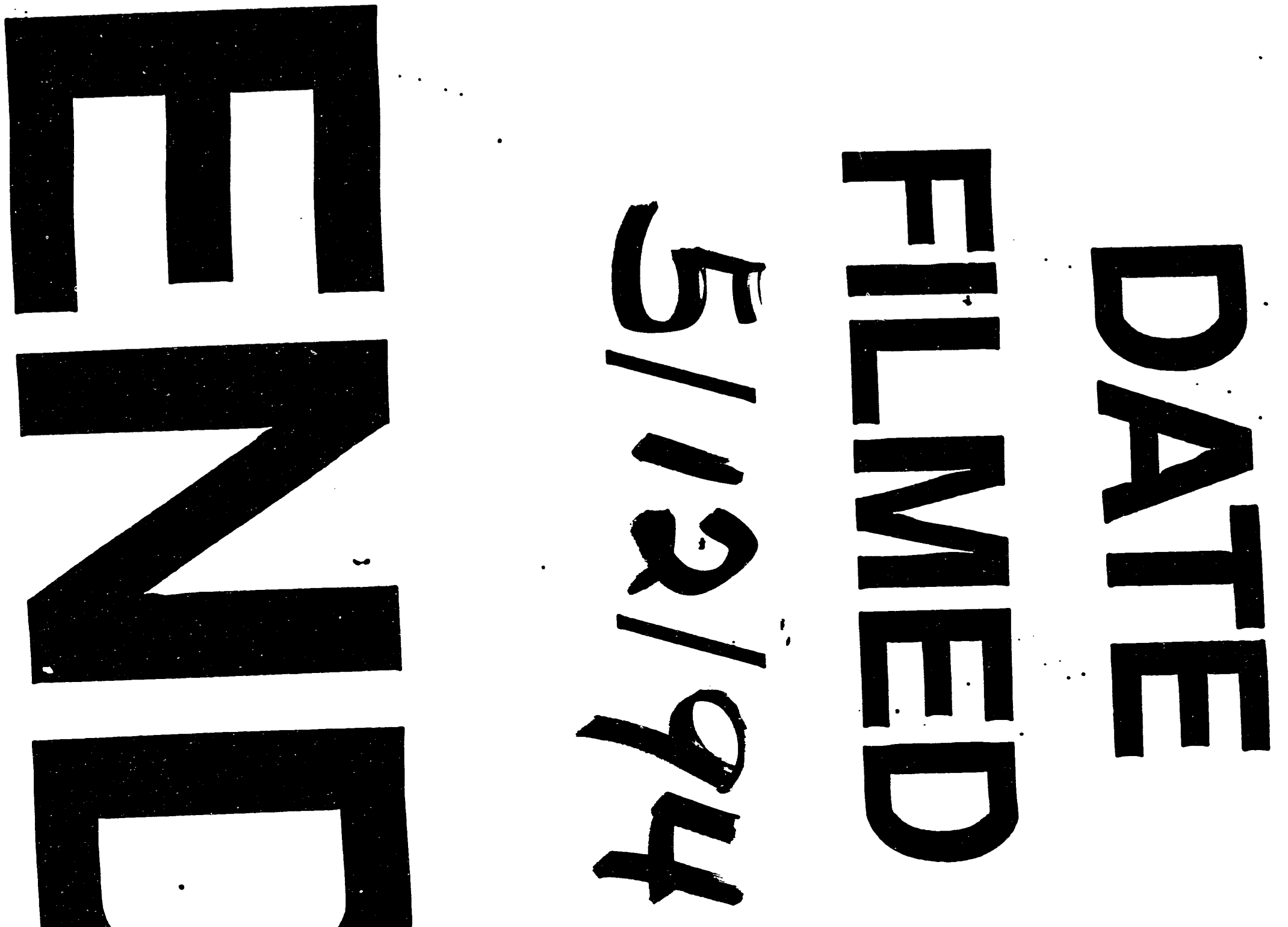


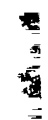

